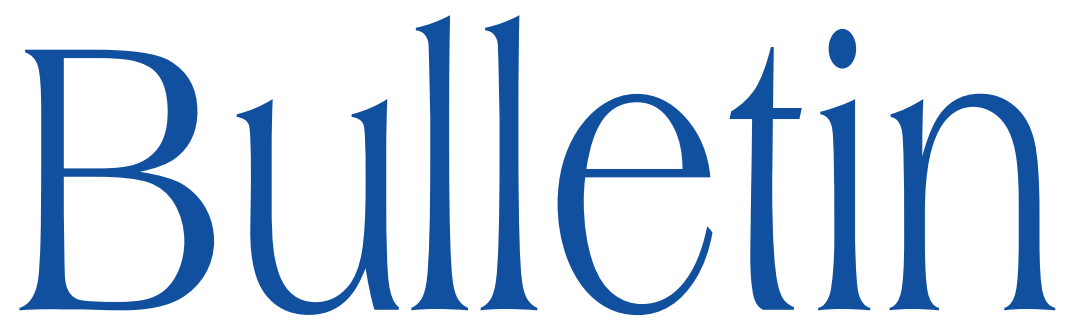

de la SOCIÉTÉ MATHÉMATIQUE DE FRANCE

\title{
UN COMPLEXE DE KOSZUL DE MODULES INSTABLES ET COHOMOTOPIE D'UN SPECTRE DE THOM
}

Nguyen Dang Ho Hai

Tome 140 Fascicule 2

2012 


\title{
UN COMPLEXE DE KOSZUL DE MODULES INSTABLES ET COHOMOTOPIE D'UN SPECTRE DE THOM
}

\author{
PAR NGuYen DANG Ho HaI
}

\begin{abstract}
RÉSumÉ. - Dans [8], les auteurs ont construit une résolution injective minimale d'un module instable dans la catégorie des modules instables modulo 2. A partir de cette résolution, un résultat de type conjecture de Segal a été obtenu pour un certain spectre de Thom. Le but de cet article est de refaire ces résultats pour les premiers impairs. Etant donné un premier impair $p$, on construit dans ce travail un complexe de Koszul dans la catégorie des modules instables sur l'algèbre de Steenrod modulo $p$. Une résolution injective d'un module instable intéressant est obtenue comme cas particulier de ce complexe de Koszul. Ce module instable est la cohomologie modulo $p$ d'un spectre de Thom qui apparaît (à $p$-complétion près) comme l'un des fibres homotopiques non contractiles dans la tour de Goodwillie du foncteur identité évaluée en la sphère $S^{3}$. Comme application de cette résolution injective, on calcule quelques groupes de cohomotopie de ce spectre à l'aide du travail de S. Zarati [24] sur les foncteurs dérivés du foncteur de déstabilisation.
\end{abstract}

Abstract (A Koszul complex of unstables modules and cohomotopy of a Thom spectrum)

We constructed in [8] a minimal injective resolution of an unstable module over the modulo 2 Steenrod algebra. From this resolution, a Segal conjecture-type result was obtained for a certain Thom spectrum. In this paper we propose to study similar problems modulo odd primes. Given $p$ an odd prime, we construct in this work a

Texte reçu le 24 mars 2011, accepté le 17 octobre 2011.

Nguyen Dang Ho HaI, Laboratoire Analyse, Géométrie et Applications, UMR 7539 du CNRS, Université Paris 13, 99, Av. J-B Clément, 93430 Villetaneuse, France Current address: Institut de Recherche en Mathématique et Physique, 2 Chemin du Cyclotron, B1348 Louvain-la-Neuve, Belgique • E-mail : nguyen@math.univ-paris13.fr

Classification mathématique par sujets (2010). - 55S10, 55Q55, $16 \mathrm{~S} 37$.

Mots clefs. - Complexe de Koszul, module instable, foncteur de déstabilisation. L'auteur est partiellement soutenu par le projet ANR blanc BLANN08-2_338236, HGRT. 
Koszul complex in the category of unstable modules over the $\bmod p$ Steenrod algebra. An injective resolution of an interesting unstable module is obtained as a special case of this Koszul complex. This unstable module is the $\bmod p$ cohomology of a Thom spectrum used in the description of the layers of the Goodwillie tower of the identity functor evaluated on the sphere $S^{3}$. As an application of the injective resolution, we compute some cohomotopy groups of the Thom spectrum using work of S. Zarati [24] on the derived functors of the destabilisation functor.

\section{Introduction}

Soient $p$ un premier et $V_{n}=(\mathbb{Z} / p)^{n}$ un $p$-groupe abélien élémentaire. On note $\rho_{n}$ la représentation réelle régulière réduite de $V_{n}$, et pour tout entier naturel $m$, on note $m \rho_{n}$ la somme directe de $m$ copies de $\rho_{n}$. On considère l'espace de Thom $B V_{n}^{m \rho_{n}}$ du fibré vectoriel associé à $m \rho_{n}$ au-dessus de l'espace classifiant $B V_{n}$. L'action naturelle du groupe linéaire général $\mathrm{GL}_{n}\left(\mathbb{F}_{p}\right)$ sur $V_{n}$ induit une action sur l'espace de Thom $B V_{n}^{m \rho_{n}}$. On pose $L(n, m)=e_{n} B V_{n}^{m \rho_{n}}$, le facteur stable de $B V_{n}^{m \rho_{n}}$ déterminé par l'idempotent de Steinberg [23] $e_{n}$ de l'anneau $\mathbb{F}_{p}\left[\mathrm{GL}_{n}\left(\mathbb{F}_{p}\right)\right]$.

Les spectres $L(n, m)$ apparaissent dans la théorie de Goodwillie comme suit. On considère la tour de Goodwillie du foncteur identité de la catégorie des espaces topologiques pointés :

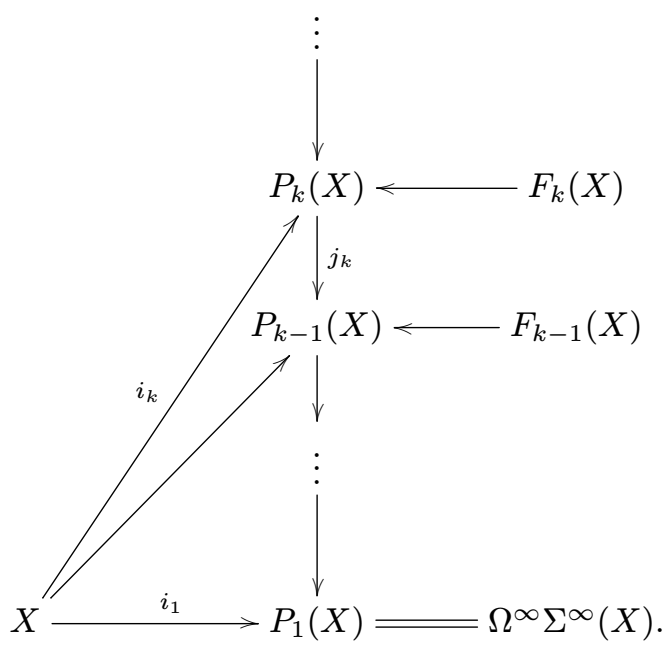

Dans ce diagramme, le foncteur $F_{k}$ est la fibre homotopique de la transformation naturelle $j_{k}: P_{k} \rightarrow P_{k-1}$. Il suit de la théorie de Goodwillie [7] que $F_{k}(X)$ 
est un espace de lacets infini, i.e. il existe un spectre $\mathscr{F}_{k}(X)$ tel que $F_{k}(X)$ soit le 0-ième espace de $\mathscr{F}_{k}(X)$ :

$$
F_{k}(X)=\Omega^{\infty} \mathscr{F}_{k}(X) .
$$

En particulier, à $p$-complétion près, si $X$ est la sphère $S^{m}(m$ est supposé impair si $p$ est impair), on a

$$
\mathscr{F}_{k}\left(S^{m}\right) \simeq \begin{cases}\Sigma^{m-n} L(n, m) & \text { si } k=p^{n} \\ * & \text { sinon. }\end{cases}
$$

(voir [3], [2, Thm. 1.9, Cor. 9.6]).

On note $L_{n, m}$ la cohomologie modulo $p$ du spectre $L(n, m)$. On se propose d'analyser $L_{n, m}$ en tant que objet de la catégorie $\mathcal{U}$ [22] des modules instables sur l'algèbre de Steenrod modulo $p, \mathscr{G}_{p}$. On sait que $L_{n, 0}$ et $L_{n, 1}$ sont des modules instables injectifs. Dans [8], on a construit explicitement une résolution injective minimale de $L_{n, 2}$ dans la catégorie des modules instables sur l'algèbre de Steenrod modulo 2. A partir de cette résolution, un résultat de type conjecture de Segal a été obtenu pour les groupes de cohomotopie du spectre $L(n, 2)$ (2-complété).

Dans cet article, on étend ces résultats à tout nombre premier impair. On profite de cette occasion d'améliorer et de clarifier la construction du complexe fondamental du cas $p=2$. Pour ce faire on introduit un complexe de Koszul approprié qui permet de généraliser les complexes exacts obtenus et ouvre de nouvelles perspectives. En particulier, en considérant la limite de ces complexes on est amené à introduire une algèbre de Koszul qui mérite une étude plus approfondie.

Dans ce qui suit, on fixe $p$ un nombre premier impair. A l'aide de l'isomorphisme de Thom, on peut considérer $L_{n, m}$ comme un sous-module de $H^{*} V_{n}$ :

$$
L_{n, m}=e_{n} \cdot\left(\mathfrak{e}_{n}^{m} H^{*} V_{n}\right) \subset H^{*} V_{n},
$$

$\mathfrak{e}_{n} \in H^{p^{n}-1} V_{n}$ étant la classe d'Euler de $\rho_{n}$. En particulier, on vérifie que $L_{n}:=L_{n, 1}$ est un facteur direct indécomposable du module instable injectif $H^{*} V_{n}$ [22], donc $L_{n}$ est un module instable injectif indécomposable.

Pour énoncer les résultats, on considère le module quotient :

$$
J_{n, m}:=\frac{L_{1}^{\otimes n}}{\sum_{i=1}^{n-1} L_{1}^{\otimes i-1} \otimes L_{2} \otimes L_{1}^{\otimes n-i-1}+L_{1}^{\otimes n-1} \otimes L_{1, m}} .
$$

On observera que $J_{n, m}$ est un module fini (voir la section 4.14). En particulier, $J_{n, 1}$ est trivial.

Voici le premier résultat de cet article. 
ThÉORÈme 1.1. - Soient $n, m$ deux entiers positifs avec $m$ impair. Il existe une suite exacte de $\mathscr{A}_{p}$-modules instables :

$$
0 \rightarrow L_{n, m} \rightarrow L_{n} \rightarrow L_{n-1} \otimes J_{1, m} \rightarrow \cdots \rightarrow L_{n-k} \otimes J_{k, m} \rightarrow \cdots \rightarrow J_{n, m} \rightarrow 0 .
$$

On verra plus bas que cette suite exacte est de type complexe de Koszul. Pour $m=1$, la suite exacte se réduit à l'identification $L_{n, 1}=L_{n}$ ci-dessus. Afin d'énoncer le résultat pour le cas $m=3$, on rappelle que le module instable de Brown-Gitler $J(i), i \in \mathbb{N}$, est un module fini caractérisé par l'isomorphisme naturel en $M \in \mathcal{U}$ :

$$
\operatorname{Hom}_{u}(M, J(i)) \cong M^{i *} \text {. }
$$

On rappelle aussi que le produit tensoriel $L_{k} \otimes J(i), k, i \in \mathbb{N}$, est un module instable injectif indécomposable [13].

THÉORÈME 1.2. - On a un isomorphisme de modules instables $J_{n, 3} \cong$ $J\left(2 p^{n}-2\right)$. En particulier, la suite exacte

$L_{n, 3} \hookrightarrow L_{n} \rightarrow L_{n-1} \otimes J(2 p-2) \rightarrow \cdots \rightarrow L_{n-k} \otimes J\left(2 p^{k}-2\right) \rightarrow \cdots \rightarrow J\left(2 p^{n}-2\right) \rightarrow 0$ est une résolution injective minimale de $L_{n, 3}$ dans la catégorie $\mathcal{U}$.

On se sert de cette résolution injective pour étudier les groupes de cohomotopie du spectre $L(n, 3)$ ( $p$-complété). Rappelons que si $X$ est un spectre, le calcul du groupe de cohomotopie $\left[X, S^{k}\right]$ des classes d'homotopie d'applications de $X$ vers le spectre des suspensions itérées de la sphère $S^{k}$ est l'une des questions importantes en topologie algébrique. Des cas particuliers ont été étudié ces dernières années parmi lesquels on peut citer la conjecture de Segal [1] concernant le calcul de $\left[\Sigma^{\infty} B V_{n}, S^{k}\right], k \geq 0$.

Pour avoir des informations sur les groupes $\left[L(n, 3), S^{k}\right]$, on utilise la suite spectrale d'Adams en cohomologie modulo $p$ dont le terme $E_{2}^{*, *} \cong$ $\operatorname{Ext}_{\mathscr{C}_{p}}^{*, *}\left(\mathbb{Z} / p, L_{n, 3}\right)$ est étudié à l'aide des foncteurs dérivés du foncteur de déstabilisation [24] et des propriétés de la résolution injective de $L_{n, 3}$ trouvée ci-dessus.

Voici un résultat partiel sur les groupes de cohomotopie du spectre $L(n, 3)$ ( $p$-complété).

ThÉORÈme 1.3. - Supposons $k \geq 2 p^{n-2}+n$. Alors

$$
\left[L(n, 3), S^{k}\right]= \begin{cases}\mathbb{Z} / p & \text { si } k=2 p^{n}-2+n, \\ \mathbb{Z} / p & \text { si } k=2 p^{n-1}-1+n \\ 0 & \text { sinon. }\end{cases}
$$

TOME $140-2012-\mathrm{N}^{\mathrm{O}} 2$ 
Plan de l'article. - Dans la section 2 on définit les modules instables $L_{n, m}$ et on donne une base additive pour chaque module $L_{n, m}$. Dans la section 3 on démontre le théorème 1.1 en construisant un complexe de Koszul associé à une suite de modules instables. Dans la section 4 on donne une présentation pour le module de Brown-Gitler $J\left(2 p^{n}-2\right)$ et ainsi démontre le théorème 1.2. Comme applications de la résolution injective de $L_{n, 3}$, on calcule quelques groupes de cohomotopie du spectre $L(n, 3)$ dans la section 5 . Pour le calcul des groupes d'extension $\operatorname{Ext}_{\mathscr{a}_{p}}^{* * *}\left(\mathbb{F}_{p}, L_{n, 3}\right)$, on aura besoin de comprendre l'espace vectoriel $\left(D(n): L_{1}\right) u$, la division dans la catégorie $\mathcal{U}$ par $L_{1}$ de $D(n):=\operatorname{Im}\left(H^{*} \Sigma_{p^{n}} \stackrel{\text { res }}{\longrightarrow}\right.$ $\left.H^{*} V_{n}\right)$. La section 6 sera consacrée à la détermination de cet espace vectoriel.

Remerciements. - Ce travail fait partie de ma thèse de doctorat effectuée à l'Université Paris 13 sous la direction de Lionel Schwartz. Je tiens à remercier Lionel Schwartz pour sa générosité, ses conseils, sa patience et ses exigences qui m'ont toujours accompagné pendant la thèse. Je remercie Geoffrey Powell pour la suggestion de l'utilisation de complexe de Koszul qui a manifestement amélioré la construction du complexe dans [8]. Enfin je remercie Saïd Zarati d'avoir écrit la thèse [24] qui joue un rôle essentiel dans l'étude des groupes de cohomotopie du spectre $L(n, 3)$.

\section{Les modules instables $L_{n, m}$}

Si $X$ est un espace ou un spectre, on note $H^{*} X$ la cohomologie modulo $p$ de $X$. La cohomologie modulo $p$ de $V_{n}$ est donnée par

$$
H^{*} V_{n} \cong \Lambda\left(x_{1}, \ldots, x_{n}\right) \otimes \mathbb{F}_{p}\left[y_{1}, \ldots, y_{n}\right],
$$

où $\left\{x_{1}, \cdots, x_{n}\right\}$ est la base canonique de l'espace dual $V_{n}^{*} \cong H^{1} V_{n}$. L'action de l'algèbre de Steenrod $\mathscr{C}_{p}$ sur $H^{*} V_{n}$ est déterminée par les formules

$$
\beta x_{i}=y_{i}, \quad P^{1} y_{i}=y_{i}^{p}, \quad 1 \leq i \leq n
$$

et la formule de Cartan. Cette action commute à l'action naturelle à gauche du semi-groupe de $n \times n$-matrices à coefficients dans $\mathbb{F}_{p}, \operatorname{Mat}_{n}\left(\mathbb{F}_{p}\right)$, sur $H^{*} V_{n}$ déterminée comme suit [18]. Si $g=\left(g_{i, j}\right) \in \operatorname{Mat}_{n}\left(\mathbb{F}_{p}\right)$ et $f \in H^{*} V_{n}$, alors

$$
(g \cdot f)\left(x_{1}, y_{1}, \cdots, x_{n}, y_{n}\right)=f\left(g \cdot x_{1}, g \cdot y_{1}, \cdots, g \cdot x_{n}, g \cdot y_{n}\right)
$$

où $g \cdot x_{j}=\sum_{i=1}^{n} g_{i, j} x_{i}$ et $g \cdot y_{j}=\sum_{i=1}^{n} g_{i, j} y_{i}$ pour $1 \leq j \leq n$.

Soit $\mathrm{GL}_{n}:=\mathrm{GL}_{n}\left(\mathbb{F}_{p}\right)$ le groupe linéaire général. L'idempotent de Steinberg $e_{n}[23]$ de l'anneau $\mathbb{F}_{p}\left[\mathrm{GL}_{n}\right]$ est défini par

$$
e_{n}=\frac{1}{\left[\mathrm{GL}_{n}: \mathrm{U}_{n}\right]} \overline{\mathrm{B}}_{n} \bar{\Sigma}_{n}
$$


Ici $\mathrm{B}_{n}$ est le sous-groupe de Borel de $\mathrm{GL}_{n}$ des matrices triangulaires supérieures, $\mathrm{U}_{n}$ le sous-groupe de Sylow des matrices triangulaires supérieures avec 1 sur la diagonale, $\Sigma_{n}$ le sous-groupe des permutations, $\overline{\mathrm{B}}_{n}=\sum_{b \in \mathrm{B}_{n}}$ et $\bar{\Sigma}_{n}=\sum_{\sigma \in \Sigma_{n}} \operatorname{sgn}(\sigma) \sigma$.

Soit $m \rho_{n}$ la somme directe de $m$ copies de la représentation réelle régulière réduite de $V_{n}$. On note $B V_{n}^{m \rho_{n}}$ l'espace de Thom du fibré vectoriel

$$
E V_{n} \times_{V_{n}} \mathbb{R}^{\left(p^{n}-1\right) m} \rightarrow B V_{n}
$$

où $V_{n}$ agit sur $\mathbb{R}^{\left(p^{n}-1\right) m}$ à travers de $m \rho_{n}$ et $E V_{n}$ est un espace contractile sur lequel $V_{n}$ agit librement. Le groupe $\mathrm{GL}_{n}$ agit sur $V_{n}$, donc agit stablement sur $B V_{n}^{m \rho_{n}}$. L'idempotent de Steinberg $e_{n}$ induit ainsi une application de spectres, notée aussi $e_{n}$, de $\Sigma^{\infty} B V_{n}^{m \rho_{n}}$ à lui-même. On a $e_{n} \circ e_{n} \simeq e_{n}$. A la suite de $\mathrm{S}$. Mitchell et S. Priddy [17], on considère le facteur stable de $B V_{n}^{m \rho_{n}}$ déterminé par $e_{n}$ :

$$
e_{n} B V_{n}^{m \rho_{n}}:=\operatorname{hocolim}\left(\Sigma^{\infty} B V_{n}^{m \rho_{n}} \stackrel{e_{n}}{\longrightarrow} \Sigma^{\infty} B V_{n}^{m \rho_{n}} \stackrel{e_{n}}{\longrightarrow} \Sigma^{\infty} B V_{n}^{m \rho_{n}} \stackrel{e_{n}}{\longrightarrow} \cdots\right) .
$$

DÉFinition 2.1. - Le spectre $L(n, m)$ et le module instable $L_{n, m}$ sont définis respectivement par $L(n, m):=e_{n} B V_{n}^{m \rho_{n}}$ et $L_{n, m}:=H^{*} L(n, m)$.

Il résulte de l'isomorphisme de Thom que

$$
L_{n, m} \cong e_{n} \cdot\left(\mathfrak{e}_{n}^{m} H^{*} V_{n}\right),
$$

où $\mathfrak{e}_{n}$ désigne la classe d'Euler de $\rho_{n}$. La classe de Chern supérieure, $\mathfrak{c}_{n}$, de la complexification $\rho_{n}^{\mathbb{C}}$ de $\rho_{n}$ est donnée par :

$$
\mathfrak{c}_{n}=(-1)^{n} \prod_{0 \neq x \in H^{1}\left(B V_{n} ; \mathbb{Z} / p\right)} \beta(x) .
$$

On vérifie que $\mathfrak{c}_{n}=\mathbf{L}_{n}^{p-1}$ où

$$
\mathbf{L}_{n}:=\left|\begin{array}{ccc}
y_{1} & \cdots & y_{n} \\
y_{1}^{p} & \cdots & y_{n}^{p} \\
\vdots & \cdots & \vdots \\
y_{1}^{p^{n-1}} & \cdots & y_{n}^{p^{n-1}}
\end{array}\right| .
$$

Comme $\mathfrak{e}_{n}^{2}=\mathfrak{c}_{n}$, on en déduit que $\mathfrak{e}_{n}=\mathbf{L}_{n}^{\frac{p-1}{2}}$ (à signe près).

On pose $M_{n}:=L_{n, 0}=e_{n} \cdot H^{*} V_{n}$ et $L_{n}:=L_{n, 1}=e_{n} \cdot\left(\mathfrak{e}_{n} H^{*} V_{n}\right)$. On observe que $M_{n}$ et $L_{n}$ sont invariants sous l'action du sous-groupe de Borel $\mathrm{B}_{n}$ de $\mathrm{GL}_{n}$.

Proposition 2.2 (Mitchell-Priddy [17]). - On a une décomposition de modules instables : $M_{n} \cong L_{n} \oplus L_{n-1}$. 
Posons $X=\frac{x}{y}, Y=y^{p-1}$, où $x$ et $y$ sont générateurs de $H^{*} \mathbb{Z} / p \cong \Lambda(x) \otimes$ $\mathbb{F}_{p}[y]$. On a $M_{1}=L_{1} \oplus \mathbb{F}_{p}$ et on vérifie que $L_{1}$ a une base additive formée par les monômes $X^{\epsilon} Y^{i}$ pour lesquels $\epsilon=0,1$ et $i>0$.

Proposition 2.3. - On a $L_{n} \cong e_{n} \cdot L_{1}^{\otimes n}$.

Pour montrer cette proposition, on a besoin d'utiliser les relations qui existent entre les idempotents de Steinberg de différents rangs. Pour $1 \leq i \leq n-1$, on désigne par $e_{2, i}$ l'image de l'idempotent $e_{2} \in \mathbb{F}_{p}\left[\mathrm{GL}_{2}\right]$ dans $\mathbb{F}_{p}\left[\mathrm{GL}_{n}\right]$ par l'inclusion canonique $\mathrm{GL}_{2} \hookrightarrow \mathrm{GL}_{n}$ en utilisant les $i$-ième et $(i+1)$-ième coordonnées.

Dans le lemme suivant, par abus de notation, $e_{n-1}$ désigne l'image de l'idempotent $e_{n-1} \in \mathbb{F}_{p}\left[\mathrm{GL}_{n-1}\right]$ dans $\mathbb{F}_{p}\left[\mathrm{GL}_{n}\right]$ par l'inclusion canonique $\mathrm{GL}_{n-1} \hookrightarrow$ $\mathrm{GL}_{n}$ en utilisant les $(n-1)$ premières coordonnées.

Lemme 2.4 ([9, 10]). - Soit $n$ un entier $\geq 2$. On a

1. $e_{n}=e_{n} e_{2, i}=e_{2, i} e_{n}$ pour tout $1 \leq i \leq n-1$;

2. $e_{n}$ est un mot de longueur maximale des idempotents $e_{2,1}, \ldots, e_{2, n-1}$;

3. $e_{n}=e_{n-1} e_{2, n-1} e_{n-1}$.

On en déduit facilement le

Corollaire 2.5. - On a

1. $e_{n}=e_{n} e_{n-1}=e_{n-1} e_{n}$,

2. $e_{n} I_{n-1} e_{n}$ est un idempotent, et

3. $I_{n-1} e_{n} I_{n-1}=e_{n-1} I_{n-1} e_{n-1}$.

Démonstration de 2.3. - Comme $M_{n}$ est un sous-module de $H^{*} V_{n}$ invariant sous l'action du groupe de Borel $\mathrm{B}_{n}$, il est aussi invariant sous l'action du tore maximal $\mathrm{GL}_{1}^{\times n}$. On en déduit que $M_{n} \subset M_{1}^{\otimes n}$, et donc $M_{n}=e_{n} \cdot M_{1}^{\otimes n}$ puisque

$$
M_{n}=e_{n} \cdot M_{n} \subset e_{n} \cdot M_{1}^{\otimes n} \subset e_{n} \cdot H^{*} B V_{n}=M_{n} .
$$

Pour montrer que $L_{n} \cong e_{n} \cdot L_{1}^{\otimes n}$, on observe d'abord que $e_{n}$ induit un endomorphisme sur $L_{1}^{\otimes n}$. A cet effet, on se ramène au cas $n=2$ en utilisant le fait que $e_{n}$ est un produit des $e_{2, i}$. Pour le cas $n=2$, on a $e_{2} \cdot L_{1}^{\otimes 2}$ est un sous-module de $M_{1}^{\otimes 2}$ qui s'écrit comme suit :

$$
M_{1} \otimes M_{1}=\left(L_{1} \otimes \mathbb{F}_{p}\right) \oplus\left(L_{1} \otimes L_{1}\right) \oplus\left(\mathbb{F}_{p} \otimes L_{1}\right) \oplus\left(\mathbb{F}_{p} \otimes \mathbb{F}_{p}\right) .
$$

L'image de $e_{2} \cdot L_{1}^{\otimes 2}$ par la projection sur $L_{1} \otimes \mathbb{F}_{p}$ (resp. $\mathbb{F}_{p} \otimes L_{1}$ ) est alors $\operatorname{diag}(1,0) e_{2} \cdot L_{1}^{\otimes 2}\left(\operatorname{resp} . \operatorname{diag}(0,1) e_{2} \cdot L_{1}^{\otimes 2}\right)$. Comme

$$
\operatorname{diag}(1,0) e_{2}=\sum_{a \in \mathbb{F}_{p}^{*}, c \in \mathbb{F}_{p}}\left(\left(\begin{array}{ll}
c & a \\
0 & 0
\end{array}\right)-\left(\begin{array}{ll}
a & c \\
0 & 0
\end{array}\right)\right)
$$

BULletin DE LA SOCIÉtÉ MATHÉmATiQUE DE FRANCE 
et $\operatorname{diag}(0,1) e_{2}=0$, il est clair que $\operatorname{diag}(1,0) e_{2}$ et $\operatorname{diag}(0,1) e_{2}$ agissent trivialement sur $L_{1}^{\otimes 2}$. On en déduit que $e_{2} \cdot L_{1}^{\otimes 2} \subset L_{1}^{\otimes 2}$.

Comme $L_{1}^{\otimes n}$ est facteur direct non trivial de $M_{1}^{\otimes n}$, le module $e_{n} \cdot L_{1}^{\otimes n}$ est facteur direct de $M_{n}=e_{n} \cdot M_{1}^{\otimes n}$. D'autre part, comme la décomposition $M_{n}=$ $L_{n} \oplus L_{n-1}$ correspond à la décomposition $e_{n}=\left(e_{n}-e_{n} I_{n-1} e_{n}\right)+e_{n} I_{n-1} e_{n}$ [11], l'image de $e_{n} \cdot L_{1}^{\otimes n}$ par la projection canonique $M_{n} \rightarrow L_{n-1}$ est $e_{n} I_{n-1} e_{n} \cdot L_{1}^{\otimes n}$. Or celui-ci est trivial car $e_{n} \cdot L_{1}^{\otimes n}$ est un sous-module de $L_{1}^{\otimes n}$ et $I_{n-1}$ agit trivialement sur ce dernier. Il suit que $e_{n} \cdot L_{1}^{\otimes n} \subset L_{n}$ et donc $e_{n} \cdot L_{1}^{\otimes n}=L_{n}$ car $L_{n}$ est indécomposable et $e_{n} \cdot L_{1}^{\otimes n}$ est non trivial. La proposition est démontrée.

Corollaire 2.6. - On a $L_{n} \cong \bigcap_{i=1}^{n-1} L_{1}^{\otimes i-1} \otimes L_{2} \otimes L_{1}^{\otimes n-i-1}$.

Démonstration. - La formule, dûe essentiellement à Kuhn ([9]), est conséquence des identifications $L_{n}=e_{n} \cdot L_{1}^{\otimes n}, L_{1}^{\otimes i-1} \otimes L_{2} \otimes L_{1}^{\otimes n-i-1}=e_{2, i} \cdot L_{1}^{\otimes n}$ et le lemme 2.4.

On décrit ensuite la structure de $L_{n}$ en tant qu'espace vectoriel gradué. Rappelons que si $I=\left(\epsilon_{1}, i_{1}, \cdots, \epsilon_{r}, i_{r}\right), P^{I}$ désigne le monôme $\beta^{\epsilon_{1}} P^{i_{1}} \cdots \beta^{\epsilon_{r}} P^{i_{r}}$ dans l'algèbre de Steenrod $\mathscr{C}_{p}$. La suite $I$ est dite admissible si $i_{j} \geq p i_{j+1}+$ $\epsilon_{j+1}$ pour tout $j$. La longueur de $I$, notée $\ell(I)$, est le plus grand entier $\ell$ tel que $\left(\epsilon_{\ell}, i_{\ell}\right) \neq(0,0)$, i.e. $I$ est de la forme $\left(\epsilon_{1}, i_{1}, \cdots, \epsilon_{\ell}, i_{\ell}, 0,0, \cdots, 0,0\right)$ avec $\left(\epsilon_{\ell}, i_{\ell}\right) \neq(0,0)$.

Pour la définition des modules $M_{n}$ et $L_{n}$, Mitchell et Priddy [17] ont considéré l'action à droite de l'idempotent $e_{n}$ sur $H^{*} V_{n}$. Ceci est équivalent à l'utilisation de l'action à gauche de l'idempotent $\tilde{e}_{n}$ défini par :

$$
\tilde{e}_{n}=\frac{1}{\left[\mathrm{GL}_{n}: U_{n}\right]} \bar{\Sigma}_{n} \overline{\mathrm{B}}_{n}
$$

c'est le conjugué de $e_{n}$ dans l'algèbre de Hopf $\mathbb{F}_{p}\left[\mathrm{GL}_{n}\right]$. On a des isomorphismes de $\mathscr{G}_{p}$-modules instables ([17, Prop. 2.6]) :

$$
e_{n} \cdot H^{*} V_{n} \stackrel{\bar{\Sigma}_{n}}{\longrightarrow} \tilde{e}_{n} \cdot H^{*} V_{n}, \quad \tilde{e}_{n} \cdot H^{*} V_{n} \stackrel{\overline{\mathrm{B}}_{n}}{\longrightarrow} e_{n} \cdot H^{*} V_{n} .
$$

Proposition 2.7 (Mitchell et Priddy [17]). — Le module $L_{n}$, identifié à $\tilde{e}_{n}$. $L_{1}^{\otimes n}$, admet pour base la famille des éléments

$$
\left\{P^{I}\left(\frac{x_{1} \cdots x_{n}}{y_{1} \cdots y_{n}}\right): I \text { admissible, } \ell(I)=n \text { et } P^{I} \notin \mathscr{G}_{p} \beta\right\} .
$$

Une base alternative de $L_{n}$ en termes d'invariants modulaires de $\mathrm{GL}_{n}$ est donnée comme suit. Rappelons d'abord les formules des invariants de Dickson

TOME $140-2012-\mathrm{N}^{\mathrm{O}} 2$ 
et Mui [18] (voir 6.1) :

$$
\mathbf{L}_{k}=\left|\begin{array}{ccc}
y_{1} & \cdots & y_{k} \\
y_{1}^{p} & \cdots & y_{k}^{p} \\
\vdots & \cdots & \vdots \\
y_{1}^{p^{k-1}} & \cdots & y_{k}^{p^{k-1}}
\end{array}\right|, \quad \mathbf{M}_{k, k-1}=\left|\begin{array}{ccc}
x_{1} & \cdots & x_{k} \\
y_{1} & \cdots & y_{k} \\
\vdots & \cdots & \vdots \\
y_{1}^{p^{k-2}} & \cdots & y_{k}^{p^{k-2}}
\end{array}\right| .
$$

Posons $\omega_{k}=\mathbf{L}_{k}^{p-1}$ (c'est l'invariant de Dickson supérieur de $\mathrm{GL}_{k}$ ) et $\mu_{k}=$ $\frac{\mathbf{M}_{k, k-1}}{\mathbf{L}_{k}}$. Le degré de $\omega_{k}$ est alors $2\left(p^{k}-1\right)$ et celui de $\mu_{k}$ est $1-2 p^{k-1}$.

Proposition 2.8. - Le module $L_{n}$, identifié à $e_{n} \cdot L_{1}^{\otimes n}$, a une base formée par les éléments

$$
e_{n} \cdot \mu_{1}^{\epsilon_{1}} \omega_{1}^{i_{1}-p i_{2}+\epsilon_{2}} \cdots \mu_{n-1}^{\epsilon_{n-1}} \omega_{n-1}^{i_{n-1}-p i_{n}+\epsilon_{n}} \cdot \mu_{n}^{\epsilon_{n}} \omega_{n}^{i_{n}}
$$

qui vérifient

$$
\epsilon_{1}, \cdots, \epsilon_{n} \in\{0,1\}, \quad i_{1}>p i_{2}-\epsilon_{2}, \quad \cdots, \quad i_{n-1}>p i_{n}-\epsilon_{n}, \quad i_{n}>0 .
$$

Démonstration. - La série de Poincaré de $L_{n}$, d'après Mitchell et Priddy [17], est donnée par

$$
P\left(L_{n}\right)=\prod_{k=1}^{n}\left(1+t^{1-2 p^{k-1}}\right) \cdot \prod_{k=1}^{n} \frac{t^{2\left(p^{k}-1\right)}}{1-t^{2\left(p^{k}-1\right)}} .
$$

Il suffit donc de vérifier que la famille des éléments dans la proposition est libre parce que $P\left(L_{n}\right)$ est également la série de Poincaré de l'espace vectoriel engendré par ces éléments. On se sert du lemme 2.11 ci-dessous pour montrer l’indépendance linéaire.

REMARque 2.9. - Dans ce qui suit, $L_{n}$ sera toujours identifié à $e_{n} \cdot L_{1}^{\otimes n}$. Ainsi $L_{n}$ est un sous-module de $H^{*} V_{n}$ invariant sous l'action du sous-groupe de Borel $B_{n}$ de $\mathrm{GL}_{n}$.

On pose $X_{i}=\frac{x_{i}}{y_{i}}$ et $Y_{i}=y_{i}^{p-1}$. On associe à chaque monôme $X_{1}^{\epsilon_{1}} Y_{1}^{i_{1}} \cdots X_{n}^{\epsilon_{n}} Y_{n}^{i_{n}}$ la suite $\left(d_{1}, \cdots, d_{n}\right)$ où $d_{j}=2(p-1) i_{j}-\epsilon_{j}$ est le degré de $X_{j}^{\epsilon_{j}} Y_{j}^{i_{j}}$. On met un ordre sur les monômes $X_{1}^{\epsilon_{1}} Y_{1}^{i_{1}} \cdots X_{n}^{\epsilon_{n}} Y_{n}^{i_{n}}$ en utilisant l'ordre antilexicographique sur les suites associées $\left(d_{1}, \cdots, d_{n}\right)$. On note que la suite $\left(d_{1}, \cdots, d_{n}\right)$ détermine de manière unique la suite $\left(\epsilon_{1}, i_{1}, \cdots, \epsilon_{n}, i_{n}\right)$.

Notation 2.10. - Soit $\left(\epsilon_{1}, i_{1}, \cdots, \epsilon_{n}, i_{n}\right)$ une suite vérifiant la condition $\left(^{*}\right)$. On pose

$$
\omega^{\epsilon_{1}, i_{1}, \cdots, \epsilon_{n}, i_{n}}=e_{n} \cdot \mu_{1}^{\epsilon_{1}} \omega_{1}^{i_{1}-p i_{2}+\epsilon_{2}} \cdots \mu_{n-1}^{\epsilon_{n-1}} \omega_{n-1}^{i_{n-1}-p i_{n}+\epsilon_{n}} \cdot \mu_{n}^{\epsilon_{n}} \omega_{n}^{i_{n}},
$$

c'est un élément de $H^{*} V_{n}$ de degré $\sum_{k=1}^{n} 2(p-1) i_{k}-\epsilon_{k}$. 
Lemme 2.11. - On a

$$
\omega^{\epsilon_{1}, i_{1}, \cdots, \epsilon_{n}, i_{n}}=\omega^{\epsilon_{1}, i_{1}, \cdots, \epsilon_{n-1}, i_{n-1}} \cdot X_{n}^{\epsilon_{n}} Y_{n}^{i_{n}}+\sum_{\operatorname{deg}\left(X_{n}^{\epsilon} Y_{n}^{i}\right)>\operatorname{deg}\left(X_{n}^{\epsilon_{n}} Y_{n}^{i_{n}}\right)} f_{\epsilon, i} \cdot X_{n}^{\epsilon} Y_{n}^{i},
$$

pour certains $f_{\epsilon, i} \in \Lambda\left(x_{1}, \ldots, x_{n-1}\right) \otimes \mathbb{F}_{p}\left[y_{1} \ldots, y_{n-1}\right]$. Par conséquent,

$$
\omega^{\epsilon_{1}, i_{1}, \cdots, \epsilon_{n}, i_{n}}=X_{1}^{\epsilon_{1}} Y_{1}^{i_{1}} \cdots X_{n}^{\epsilon_{n}} Y_{n}^{i_{n}}+\text { des monômes d'ordre supérieur. }
$$

Démonstration. - Posons

$$
Q:=Q\left(x_{1}, y_{1}, \cdots, x_{n-1}, y_{n-1}\right)=\mu_{1}^{\epsilon_{1}} \omega_{1}^{i_{1}-p i_{2}+\epsilon_{2}} \cdots \mu_{n-1}^{\epsilon_{n-1}} \omega_{n-1}^{i_{n-1}-p i_{n}+\epsilon_{n}} .
$$

Notons d'abord que $\mu_{n}^{\epsilon_{n}} \omega_{n}^{i_{n}}$ est $\mathrm{GL}_{n}$-invariant et l'on a un développement

$$
\mu_{n}^{\epsilon_{n}} \omega_{n}^{i_{n}}=\omega_{n-1}^{p i_{n}-\epsilon_{n}} \cdot X_{n}^{\epsilon_{n}} Y_{n}^{i_{n}}+\sum_{\operatorname{deg}\left(X_{n}^{\epsilon} Y_{n}^{i}\right)>\operatorname{deg}\left(X_{n}^{\epsilon_{n}} Y_{n}^{i_{n}}\right)} g_{\epsilon, i} \cdot X_{n}^{\epsilon} Y_{n}^{i}
$$

pour certains $g_{\epsilon, i} \in \Lambda\left(x_{1}, \ldots, x_{n-1}\right) \otimes \mathbb{F}_{p}\left[y_{1}, \ldots, y_{n-1}\right]$. Il suffit donc de montrer que

$$
e_{n} \cdot Q=e_{n-1} \cdot Q+\sum_{\operatorname{deg}\left(X_{n}^{\epsilon} Y_{n}^{i}\right)>0} h_{\epsilon, i} \cdot X_{n}^{\epsilon} Y_{n}^{i}
$$

pour certains $h_{\epsilon, i} \in \Lambda\left(x_{1}, \cdots, x_{n-1}\right) \otimes \mathbb{F}_{p}\left[y_{1}, \cdots, y_{n-1}\right]$. De manière équivalente, il suffit de montrer que

$$
I_{n-1} e_{n} \cdot Q=e_{n-1} \cdot Q
$$

avec $I_{n-1}=\operatorname{diag}\left(1^{n-1}, 0\right)$. En effet, comme $Q$ et $e_{n-1} \cdot Q$ ne font intervenir que les variables $x_{1}, y_{1}, \ldots, x_{n-1}, y_{n-1}$, ils sont invariants sous l'action de $I_{n-1}$. Il suit que

$$
\begin{aligned}
I_{n-1} e_{n} \cdot Q & =I_{n-1} e_{n} I_{n-1} \cdot Q & & \left(\operatorname{car} Q=I_{n-1} \cdot Q\right) \\
& =e_{n-1} I_{n-1} e_{n-1} \cdot Q & & \left(\operatorname{car} I_{n-1} e_{n} I_{n-1}=e_{n-1} I_{n-1} e_{n-1}\right) \\
& =e_{n-1} e_{n-1} \cdot Q & & \left(\operatorname{car} I_{n-1} e_{n-1} \cdot Q=e_{n-1} \cdot Q\right) \\
& =e_{n-1} \cdot Q . & &
\end{aligned}
$$

Le lemme est démontré.

Corollaire 2.12. - Soient $n$ et $m$ deux entiers positifs avec $m$ impair. Alors $L_{n, m}$ a une base formée par les éléments

$$
e_{n} \cdot \mu_{1}^{\epsilon_{1}} \omega_{1}^{i_{1}-p i_{2}+\epsilon_{2}} \cdots \mu_{n-1}^{\epsilon_{n-1}} \omega_{n-1}^{i_{n-1}-p i_{n}+\epsilon_{n}} \cdot \mu_{n}^{\epsilon_{n}} \omega_{n}^{i_{n}}
$$

qui vérifient $\epsilon_{1}, \cdots, \epsilon_{n} \in\{0,1\}, i_{1}>p i_{2}-\epsilon_{2}, \ldots, i_{n-1}>p i_{n}-\epsilon_{n}$ et $i_{n}>\frac{m-1}{2}$. De plus, on a $L_{n, m}=L_{n} \cap\left(L_{1}^{\otimes n-1} \otimes L_{1, m}\right)$

Démonstration. - On a $L_{n, m}=\mathfrak{e}_{n}^{m-1} L_{n}$ car $\mathfrak{e}_{n}^{2}$ est $\mathrm{GL}_{n}$-invariant. D'autre part, d'après le lemme 2.11, un élément de base $\omega^{\epsilon_{1}, i_{1}, \cdots, \epsilon_{n}, i_{n}} \in L_{n}$ est un élément de $L_{n, m}$ si et seulement si $i_{n}>\frac{m-1}{2}$. Le corollaire suit. 


\section{Un complexe de Koszul de modules instables}

Dans cette section on démontre le théorème 1.1 en introduisant un complexe de Koszul approprié.

3.1. Complexe de Koszul associé à une suite d'espaces vectoriels. - Soit $M$ un $\mathbb{F}_{p}$-espace vectoriel gradué et $\left\{M_{i}\right\}_{i \in \mathbb{Z}}$ une suite de sous-espaces de $M$. Pour chaque $s$, considérons le module quotient

$$
K(M)_{s}=\frac{\bigcap_{i \leq s-1} M_{i}}{\left(\bigcap_{i \leq s-1} M_{i}\right) \cap\left(\sum_{i \geq s+1} M_{i}\right)}
$$

Notons $d(M)_{s}: K(M)_{s} \rightarrow K(M)_{s-1}$ le morphisme naturel

$$
\frac{\bigcap_{i \leq s-1} M_{i}}{\left(\bigcap_{i \leq s-1} M_{i}\right) \cap\left(\sum_{i \geq s+1} M_{i}\right)} \longrightarrow \frac{\bigcap_{i \leq s-2} M_{i}}{\left(\bigcap_{i \leq s-2} M_{i}\right) \cap\left(\sum_{i \geq s} M_{i}\right)} .
$$

Il est clair que le morphisme composé $d(M)_{s} \circ d(M)_{s+1}$ :

$$
\frac{\bigcap_{i \leq s} M_{i}}{\left(\bigcap_{i \leq s} M_{i}\right) \cap\left(\sum_{i \geq s+2} M_{i}\right)} \rightarrow \frac{\bigcap_{i \leq s-1} M_{i}}{\left(\bigcap_{i \leq s-1} M_{i}\right) \cap\left(\sum_{i \geq s+1} M_{i}\right)} \rightarrow \frac{\bigcap_{i \leq s-2} M_{i}}{\left(\bigcap_{i \leq s-2} M_{i}\right) \cap\left(\sum_{i \geq s} M_{i}\right)}
$$

est trivial pour tout $s$. On obtient ainsi un complexe dit complexe de Koszul associé à la suite $\left\{M_{i}\right\}_{i \in \mathbb{Z}}$ de sous-espaces de $M$ :

$$
\cdots \rightarrow K(M)_{s+1} \stackrel{d(M)_{s+1}}{\longrightarrow} K(M)_{s} \stackrel{d(M)_{s}}{\longrightarrow} K(M)_{s-1} \rightarrow \cdots
$$

Proposition 3.1 (Cf. [20, Prop. 7.2, p. 16]). - Le complexe $\mathcal{K}(M)_{*}$ est exact si et seulement si, pour tout $s$, on a

$$
\left(\bigcap_{i \leq s-1} M_{i}\right) \cap\left(\sum_{i \geq s} M_{i}\right)=\bigcap_{i \leq s} M_{i}+\left(\bigcap_{i \leq s-1} M_{i}\right) \cap\left(\sum_{i \geq s+1} M_{i}\right) .
$$

Démonstration. - On pose $A_{s}=\bigcap_{i \leq s} M_{i}$ et $B_{s}=\sum_{i \geq s} M_{i}$. On observe que $A_{s} \subset A_{s-1}, B_{s} \subset B_{s-1}$ et $A_{s} \subset B_{s}$ pour tout $s$. Par définition, on a $K(M)_{s}=$ $A_{s-1} / A_{s-1} \cap B_{s+1}$. Le noyau de $d(M)_{s}: A_{s-1} / A_{s-1} \cap B_{s+1} \rightarrow A_{s-2} / A_{s-2} \cap B_{s}$ est alors donné par

$$
\operatorname{ker} d(M)_{s}=\left(A_{s-1} \cap B_{s}\right) /\left(A_{s-1} \cap B_{s+1}\right) .
$$

Comme $\operatorname{Im} d(M)_{s+1} \cong K_{s+1} / \operatorname{ker} d_{s+1}$, on a

$$
\begin{aligned}
\operatorname{Im} d(M)_{s+1} & \cong A_{s} /\left(A_{s} \cap B_{s+1}\right) \\
& \cong A_{s} /\left(A_{s} \cap A_{s-1} \cap B_{s+1}\right) \quad\left(\operatorname{car} A_{s} \subset A_{s-1}\right) \\
& \cong\left(A_{s}+A_{s-1} \cap B_{s+1}\right) /\left(A_{s-1} \cap B_{s+1}\right) .
\end{aligned}
$$


L'inclusion $\operatorname{Im} d(M)_{s+1} \hookrightarrow \operatorname{ker} d(M)_{s}$ est alors induite par l'inclusion naturelle $\left(A_{s}+A_{s-1} \cap B_{s+1}\right) \subset\left(A_{s-1} \cap B_{s}\right)$. On en déduit que le complexe est exact si et seulement si $A_{s-1} \cap B_{s}=A_{s}+A_{s-1} \cap B_{s+1}$ pour tout $s$. La proposition est démontrée.

3.2. Démonstration de l'exactitude du complexe. - Pour démontrer le théorème 1.1 , on considère la suite des sous-modules instables $\left\{\mathfrak{M}_{i}\right\}_{i \in \mathbb{Z}}$ de $L_{1}^{\otimes n}$ définie par

$$
\mathfrak{M}_{i}= \begin{cases}L_{1}^{\otimes n} & \text { si } i \leq 0, \\ L_{1}^{\otimes i-1} \otimes L_{2} \otimes L_{1}^{\otimes n-i-1} & \text { si } 1 \leq i \leq n-1, \\ L_{1}^{\otimes n-1} \otimes L_{1, m} & \text { si } i=n, \\ 0 & \text { si } i>n .\end{cases}
$$

Par définition, $J_{n, m}$ est donné par le quotient :

$$
J_{n, m}=\frac{L_{1}^{\otimes n}}{\mathfrak{M}_{1}+\cdots+\mathfrak{M}_{n}} .
$$

Le complexe $\mathcal{K}(\mathfrak{M})_{*}$ est en fait le complexe

$$
0 \rightarrow L_{n, m} \rightarrow L_{n} \rightarrow L_{n-1} \otimes J_{1, m} \rightarrow \cdots \rightarrow L_{n-s} \otimes J_{s, m} \rightarrow \cdots \rightarrow J_{n, m} \rightarrow 0
$$

du théorème 1.1 grâce à l'identification suivante.

Proposition 3.2. - On a

$$
K(\mathfrak{M})_{s} \cong \begin{cases}L_{s} \otimes J_{n-s, m} & \text { si } 0 \leq s \leq n \\ L_{n, m} & \text { si } s=n+1 \\ 0 & \text { sinon. }\end{cases}
$$

Démonstration. - Posons $A_{s}=\bigcap_{i \leq s} \mathfrak{M}_{i}, B_{s}=\sum_{i \geq s} \mathfrak{M}_{i}$ et $K_{s}:=K(\mathfrak{M})_{s}$. On a $K_{s}=A_{s-1} / A_{s-1} \cap B_{s+1}$. On considère les cas suivants :

1. Si $s<0$, on a $K_{s}=0$ car $A_{s}=B_{s}=L_{1}^{\otimes n}$ si $s \leq 0$.

2. Si $s=0$, on a $K_{0}=A_{-1} /\left(A_{-1} \cap B_{1}\right)=L_{1}^{\otimes n} / \sum_{i=1}^{n} \mathfrak{M}_{i}=J_{n, m}$.

3. Si $0<s<n$, d'après le corollaire 2.6 , on a

$$
A_{s-1}=\bigcap_{i=1}^{s-1} \mathfrak{M}_{i}=L_{s} \otimes L_{1}^{\otimes n-s}
$$

et

$$
B_{s+1}=L_{1}^{\otimes s} \otimes\left(\sum_{i=1}^{n-s-1} L_{1}^{\otimes i-1} \otimes L_{2} \otimes L_{1}^{n-s-i-1}+L_{1}^{\otimes n-s-1} \otimes L_{1, m}\right) .
$$

On en déduit que $K_{s}=A_{s-1} /\left(A_{s-1} \cap B_{s+1}\right) \cong L_{s} \otimes J_{n-s, m}$.

4. Si $s=n$, on a $K_{n}=A_{n-1} /\left(A_{n-1} \cap B_{n+1}\right)=A_{n-1}=L_{n}$ à l'aide du corollaire 2.6. 
5. Si $s=n+1$, l'égalité $L_{n, m}=\bigcap_{i=1}^{n} \mathfrak{M}_{i}$ est équivalent à l'égalité $L_{n, m}=$ $L_{n} \cap\left(L_{1}^{\otimes n-1} \otimes L_{1, m}\right):$ c'est le corollaire 2.12.

6. Si $s>n+1, K_{s}$ est trivial car $A_{s}=B_{s}=0$ si $s>n$.

La proposition est démontrée.

La démonstration du théorème 1.1 est achevée en utilisant la proposition 3.1 et la proposition suivante.

Proposition 3.3. - Pour tout $0<s<n$, on a

$$
\left(\bigcap_{i=1}^{s-1} \mathfrak{M}_{i}\right) \cap\left(\sum_{i=s}^{n} \mathfrak{M}_{i}\right)=\bigcap_{i=1}^{s} \mathfrak{M}_{i}+\left(\bigcap_{i=1}^{s-1} \mathfrak{M}_{i}\right) \cap\left(\sum_{i=s+1}^{n} \mathfrak{M}_{i}\right)
$$

Afin de démontrer cette proposition, on a besoin d'introduire quelques notations.

Notation 3.4. - Soit $I=\left(\epsilon_{1}, i_{1}, \cdots, \epsilon_{n}, i_{n}\right)$ et soit $S=\left(s_{1}, \cdots, s_{k}\right)$ avec $s_{1}+\cdots+s_{k}=n$. On note $r_{0}=1, r_{j}=s_{1}+\cdots+s_{j}, 1 \leq j \leq k$, et on suppose que la suite $\left(\epsilon_{r_{j-1}}, i_{r_{j-1}}, \cdots, \epsilon_{r_{j}}, i_{r_{j}}\right), 1 \leq j \leq k$, vérifie la condition :

$\epsilon_{r_{j-1}}, \ldots, \epsilon_{r_{j}} \in\{0,1\}, \quad i_{r_{j-1}}>p i_{r_{j-1}+1}-\epsilon_{r_{j-1}}, \cdots, i_{r_{j}-1}>p i_{r_{j}}-\epsilon_{r_{j}}, \quad i_{r_{j}}>0$.

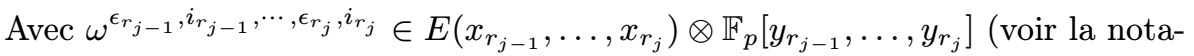
tion 2.10), on pose

$$
\omega_{S}^{I}:=\prod_{j=1}^{k} \omega^{\epsilon_{r_{j-1}}, i_{r_{j-1}}, \cdots, \epsilon_{r_{j}}, i_{r_{j}}} .
$$

On appelle $\omega_{S}^{I}$ un $S$-monôme. On associe à chaque $S$-monôme $\omega_{S}^{I}$ la suite $\left(d_{1}, \cdots, d_{n}\right)$ où $d_{j}=2(p-1) i_{j}-\epsilon_{j}$ est le degré de $X_{j}^{\epsilon_{j}} Y_{j}^{i_{j}}$. On met un ordre sur les $S$-monômes en utilisant l'ordre anti-lexicographique sur les suites associées $\left(d_{1}, \cdots, d_{n}\right)$.

Comme titre d'exemple, explicitons quelques $S$-monômes :

$$
\begin{gathered}
\omega_{(n)}^{I}=\omega^{\epsilon_{1}, i_{1}, \cdots, \epsilon_{n}, i_{n}}, \quad \omega_{\left(1^{n}\right)}^{I}=X_{1}^{\epsilon_{1}} Y_{1}^{i_{1}} \cdots X_{n}^{\epsilon_{n}} Y_{n}^{i_{n}}, \\
\omega_{\left(s, 1^{n-s}\right)}^{I}=\omega^{\epsilon_{1}, i_{1}, \cdots, \epsilon_{s}, i_{s}} X_{s+1}^{\epsilon_{s+1}} Y_{s+1}^{i_{s+1}} \cdots X_{n}^{\epsilon_{n}} Y_{n}^{i_{n}} .
\end{gathered}
$$

Démonstration de la proposition 3.3. - La démonstration s'appuie essentiellement sur le lemme 2.11. Posons

$$
\begin{aligned}
\text { LHS } & :=\left(\bigcap_{i=1}^{s-1} \mathfrak{M}_{i}\right) \cap\left(\sum_{i=s}^{n} \mathfrak{M}_{i}\right), \\
\text { RHS } & :=\bigcap_{i=1}^{s} \mathfrak{M}_{i}+\left(\bigcap_{i=1}^{s-1} \mathfrak{M}_{i}\right) \cap\left(\sum_{i=s+1}^{n} \mathfrak{M}_{i}\right) .
\end{aligned}
$$


L'inclusion LHS $\supset$ RHS est évidente. Montrons l'inclusion LHS $\subset$ RHS. Soit $f$ un élément homogène de LHS. Comme $\bigcap_{i=1}^{s-1} \mathfrak{M}_{i}=L_{s} \otimes L_{1}^{\otimes n-s}, f$ est une combinaison de $\left(s, 1^{n-s}\right)$-monômes $\omega_{\left(s, 1^{n-s}\right)}^{I}$. Un tel monôme est dit $s$-acceptable si $1 \leq i_{j} \leq p i_{j+1}-\epsilon_{j+1}$ pour $s \leq j<n$ et $1 \leq i_{n} \leq \frac{m-1}{2}$.

Supposons que $\omega_{\left(s, 1^{n-s}\right)}^{I}$ n'est pas $s$-acceptable. Considérons les cas suivants :

- Si $i_{n}>\frac{m-1}{2}$, alors $\omega_{\left(s, 1^{n-s}\right)}^{I} \in \mathfrak{M}_{n}$, donc $\omega_{\left(s, 1^{n-s}\right)}^{I} \in$ RHS.

- Si $i_{j}>p i_{j+1}-\epsilon_{j+1}$ pour $s<j<n$, d'après le lemme 2.11, on a $\omega_{\left(s, 1^{j-1-s}, 2,1^{n-j-1}\right)}^{I}=\omega_{\left(s, 1^{n-s}\right)}^{I}+\operatorname{des}\left(s, 1^{n-s}\right)$-monômes d'ordre supérieur, et $\omega_{\left(s, 1^{j-1-s}, 2,1^{n-j-1}\right)}^{I}$ est un élément de $\mathfrak{M}_{j}$, donc appartient à RHS.

- Si $i_{s}>p i_{s+1}-\epsilon_{s+1}$, d'après le lemme 2.11, on a $\omega_{\left(s+1,1^{n-s-1}\right)}^{I}=\omega_{\left(s, 1^{n-s}\right)}^{I}+\operatorname{des}\left(s, 1^{n-s}\right)$-monômes d'ordre supérieur, et $\omega_{\left(s+1,1^{n-s-1}\right)}^{I}$ appartient à $\bigcap_{i=1}^{s} \mathfrak{M}_{i}$, donc $\omega_{\left(s+1,1^{n-s-1}\right)}^{I} \in \mathrm{RHS}$.

Une itération évidente permet alors d'écrire $f$ sous la forme $f=f^{\prime} \bmod$ RHS, où $f^{\prime}$ est une somme de monômes $s$-acceptables $\omega_{\left(s, 1^{n-s}\right)}^{I}$. Montrons $f^{\prime}=0$. Supposons au contraire que $f^{\prime} \neq 0$. Soit $X_{1}^{\epsilon_{1}} Y_{1}^{i_{1}} \cdots X_{n}^{\epsilon_{n}} Y_{n}^{i_{n}}$ le monôme d'ordre minimal qui apparaît dans l'expression de $f^{\prime}$ comme combinaison linéaire de monômes distincts. On a $1 \leq i_{j} \leq p i_{j+1}-\epsilon_{j+1}$ pour $s \leq j<n$ et $1 \leq i_{n} \leq \frac{m-1}{2}$.

D'autre part, comme RHS $\subset \sum_{i=s}^{n} \mathfrak{M}_{i}$ et $f \in \sum_{i=s}^{n} \mathfrak{M}_{i}$, il suit que $f^{\prime} \in$ $\sum_{i=s}^{n} \mathfrak{M}_{i}$. Il existe alors $s \leq j \leq n$ tel que le monôme $X_{1}^{\epsilon_{1}} Y_{1}^{i_{1}} \cdots X_{n}^{\epsilon_{n}} Y_{n}^{i_{n}}$ provient de certain élément de base de $\mathfrak{M}_{j}$. On exclut le cas $j=n$ car $i_{n} \leq \frac{m-1}{2}$. Supposons que $s \leq j \leq n-1$. Alors $X_{1}^{\epsilon_{1}} Y_{1}^{i_{1}} \cdots X_{n}^{\epsilon_{n}} Y_{n}^{i_{n}}$ provient de l'élément de base $\omega_{\left(1^{j-1}, 2,1^{n-j-1}\right)}^{I}$ de $\mathfrak{M}_{j}$. Il suit que $i_{j}>p i_{j+1}-\epsilon_{j+1}$ ce qui contradit la condition $1 \leq i_{j} \leq p i_{j+1}-\epsilon_{j+1}$ ci-dessus. Le résultat suit.

3.3. Limite projective du complexe. - Considérons la limite projective

$$
J_{n, \infty}:=\lim _{\longleftarrow}\left(J_{n, 1} \nleftarrow J_{n, 3} \nleftarrow J_{n, 5} \nleftarrow \cdots\right) .
$$

La définition des modules $J_{n, m}$ implique

$$
J_{n, \infty}=\frac{L_{1}^{\otimes n}}{\sum_{i=1}^{n-1} L_{1}^{\otimes i-1} \otimes L_{2} \otimes L_{1}^{\otimes n-i-1}} .
$$

En posant $\hat{e}_{2}=1-e_{2}$, on obient une involution sur la sous-algèbre de $\mathbb{F}_{p}\left[\mathrm{GL}_{n}\left(\mathbb{F}_{p}\right)\right]$ engendrée par 1 et les idempotents $e_{2,1}, \ldots, e_{2, n-1}$. De manière récurrente, on a $\hat{e}_{2, i}=1-e_{2, i}$ et $\hat{e}_{n}=\hat{e}_{n-1} \hat{e}_{2, n-1} \hat{e}_{n-1}$ pour $n \geq 2$.

On pose $\hat{L}_{0}=\mathbb{F}_{p}, \hat{L}_{1}=L_{1}$ et $\hat{L}_{n}=\hat{e}_{n} \cdot L_{1}^{\otimes n}$ pour $n \geq 2$. On vérifie, comme dans le corollaire 2.6, que

$$
\hat{L}_{n}=\bigcap_{i=1}^{n-1} L_{1}^{\otimes i-1} \otimes \hat{L}_{2} \otimes L_{1}^{n-i-1} .
$$

TOME $140-2012-\mathrm{N}^{\mathrm{O}} 2$ 
Le résultat suivant provient de [9].

Proposition 3.5. - On a un isomorphisme de modules instables $J_{n, \infty} \cong \hat{L}_{n}$.

Démonstration. - Soit $\gamma_{n}: L_{1}^{\otimes n} \rightarrow \hat{L}_{n}$ la projection canonique. Comme $\hat{e}_{n} e_{2, i}=0$, le noyau de $\gamma_{n}$ contient la somme $\sum_{i=1}^{n-1} L_{1}^{\otimes i-1} \otimes L_{2} \otimes L_{1}^{\otimes n-i-1}$. On vérifie l'inclusion inverse. Soit $u \in L_{1}^{\otimes n}$ un élément du noyau de $\gamma_{n}$. On a $\hat{e}_{n} \cdot u=0$. Comme $\hat{e}_{n}$ est un produit des $\hat{e}_{2, i}, 1 \leq i \leq n-1$, on peut écrire $\hat{e}_{n}=\hat{e}_{2, i_{1}} \cdots \hat{e}_{2, i_{k}}$ avec $1 \leq i_{j} \leq n-1$. On a

$$
\begin{aligned}
1 & =\hat{e}_{2, i_{1}} \cdots \hat{e}_{2, i_{k}}+\left(1-\hat{e}_{2, i_{1}}\right) \hat{e}_{2, i_{2}} \cdots \hat{e}_{2, i_{k}}+\cdots+\left(1-\hat{e}_{2, i_{k-1}}\right) \hat{e}_{2, i_{k}}+\left(1-\hat{e}_{2, i_{k}}\right) \\
& =\hat{e}_{2, i_{1}} \cdots \hat{e}_{2, i_{k}}+e_{2, i_{1}} \hat{e}_{2, i_{2}} \cdots \hat{e}_{2, i_{k}}+\cdots+e_{2, i_{k-1}} \hat{e}_{2, i_{k}}+e_{2, i_{k}} .
\end{aligned}
$$

Comme $\hat{e}_{2, i_{1}} \cdots \hat{e}_{2, i_{k}} \cdot u=0$, on a

$$
u=e_{2, i_{1}} \hat{e}_{2, i_{2}} \cdots \hat{e}_{2, i_{k}} \cdot u+\cdots+e_{2, i_{k-1}} \hat{e}_{2, i_{k}} \cdot u+e_{2, i_{k}} \cdot u,
$$

ce qui entraîne que $u$ est un élément de $\sum_{j=1}^{k} e_{2, i_{j}} \cdot L_{1}^{\otimes n}$. La proposition suit.

COROLlaire 3.6. - On a une suite exacte de modules instables injectifs réduits :

$$
0 \rightarrow L_{n} \rightarrow L_{n-1} \otimes \hat{L}_{1} \rightarrow L_{n-2} \otimes \hat{L}_{2} \rightarrow \cdots \rightarrow L_{n-k} \otimes \hat{L}_{k} \rightarrow \cdots \rightarrow \hat{L}_{n} \rightarrow 0 .
$$

Dans ce corollaire, le produit tensoriel $L_{n-k} \otimes \hat{L}_{k}, 0 \leq k \leq n$, est un module instable injectif car il est le facteur direct de $L_{1}^{\otimes n}$ associé à l'idempotent $e_{n-k} \otimes$ $\hat{e}_{k}$.

A la suite de [21], on appelle algèbre de pré-Koszul homogène une algèbre de la forme Tens $(V) /(I)$ où $V$ est un $\mathbb{F}_{p}$-espace vectoriel gradué de type fini et $(I)$ l'idéal de l'algèbre tensorielle Tens $(V)$ engendré par un sous-espace $I$ de $V \otimes V$. Une algèbre de pré-Koszul homogène $A$ est une algèbre de Koszul homogène si l'algèbre $\operatorname{Ext}_{A}^{*}\left(\mathbb{F}_{p}, \mathbb{F}_{p}\right)$ est engendrée par les éléments de $\operatorname{Ext}_{A}^{1}\left(\mathbb{F}_{p}, \mathbb{F}_{p}\right)$.

Soit $A=\operatorname{Tens}(V) /(I), I \subset V \otimes V$, une algèbre de pré-Koszul. On pose $K_{0}=\mathbb{F}_{p}, K_{1}=V$ et $K_{n}=\bigcap_{i=1}^{n-1} V^{\otimes i-1} \otimes I \otimes V^{\otimes n-i-1}$ pour $n \geq 2$. Pour tout $n$, l'inclusion naturelle $K_{n} \hookrightarrow K_{n-1} \otimes V$ définit de manière évidente un morphisme $d: K_{n} \otimes A \rightarrow K_{n-1} \otimes A$. On vérifie que $d^{2}=0$ et on obtient ainsi un complexe de $A$-modules libres à gauche $\left\{K_{n} \otimes A\right\}_{n \geq 0}$. D'après [15, Theorem 1.2], $A$ est une algèbre de Koszul homogène si et seulement si ce complexe est une résolution libre pour le module trivial $\mathbb{F}_{p}$.

A l'aide du corollaire 3.6, on obtient le

Corollaire 3.7. - L'algèbre $\bigoplus_{n \geq 0} \hat{L}_{n} \cong \operatorname{Tens}\left(L_{1}\right) /\left(L_{2}\right)$ est une algèbre de Koszul homogène. 


\section{Résolution injective minimale de $L_{n, 3}$}

Dans cette section, on démontre le théorème 1.2. On rappelle que le module $L_{1}$ a une base formée par les éléments $X^{\epsilon} Y^{i}$ avec $\epsilon=0,1$ et $i \geq 1$. Ici $X=\frac{x}{y}$ et $Y=y^{p-1}, x$ et $y$ sont des générateurs de $H^{*} \mathbb{Z} / p=\Lambda(x) \otimes \mathbb{F}_{p}[y]$.

Rappelons que pour tout $k \in \mathbb{N}$, le module instable injectif de Brown-Gitler $J(k)$ est caractérisé par l'isomorphisme naturel :

$$
\operatorname{Hom}_{u}(M, J(k))=\left(M^{k}\right)^{*}, \quad M \in \mathcal{U} .
$$

On note $\pi_{i}: L_{1} \rightarrow J\left(2 p^{i+1}-2 p^{i}\right)$ le morphisme qui envoie $Y^{p^{i}}$ sur la classe canonique $\iota_{2 p^{i+1}-2 p^{i}}$ de $J\left(2 p^{i+1}-2 p^{i}\right)$. On considère le morphisme composé

$$
g_{n}: L_{1}^{\otimes n} \stackrel{\pi_{n-1} \otimes \cdots \otimes \pi_{0}}{\longrightarrow} J\left(2 p^{n}-2 p^{n-1}\right) \otimes \cdots \otimes J(2 p-2) \stackrel{\mu}{\longrightarrow} J\left(2 p^{n}-2\right),
$$

avec $\mu$ la multiplication de modules de Brown-Gitler. Dans cette section, on notera $\mathfrak{M}_{i}=L_{1}^{\otimes i-1} \otimes L_{2} \otimes L_{1}^{\otimes n-i-1}, 1 \leq i \leq n-1$, et $\mathfrak{M}_{n}=L_{1}^{\otimes n-1} \otimes L_{1,3}$.

Pour démontrer le théorème 1.2, il suffit de démontrer le théorème suivant.

THÉORÈmE 4.1. - Le morphisme $g_{n}$ est surjectif et induit un isomorphisme de modules instables :

$$
\frac{L_{1}^{\otimes n}}{\mathfrak{M}_{1}+\cdots+\mathfrak{M}_{n}} \cong J\left(2 p^{n}-2\right) .
$$

Démonstration. - La section 4.1 montre que le noyau de $g_{n}$ contient la somme $\mathfrak{M}_{1}+\cdots+\mathfrak{M}_{n}$. Dans la section 4.3 , on trouvera que, en chaque degré, la dimension du module quotient est inférieure ou égale à celle du module de Brown-Gitler $J\left(2 p^{n}-2\right)$. Enfin la surjectivité de $g_{n}$ (le point le plus difficile de l'argument) donnée dans la section 4.2, permet de conclure le théorème.

Remarque 4.2. - En supposant ce théorème, le morphisme

$$
f_{k, n}: L_{n-k} \otimes J\left(2 p^{k}-2\right) \rightarrow L_{n-k-1} \otimes J\left(2 p^{k+1}-2\right)
$$

n'est rien d'autre que le morphisme pointillé dans le diagramme commutatif suivant :

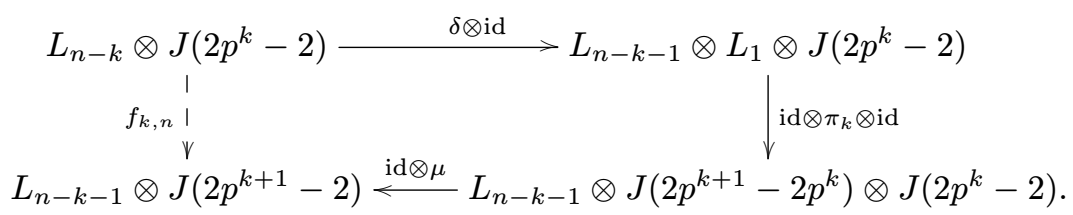

Le diagonal $\delta: L_{n} \rightarrow L_{i} \otimes L_{n-i}$ dans ce diagramme est l'inclusion canonique induite de l'identification $L_{n}=e_{n} \cdot L_{1}^{\otimes n}$ et de l'identité $e_{n}=\left(e_{i} \otimes e_{n-i}\right) e_{n}, e_{i}$ (resp. $e_{n-i}$ ) agissant sur les $i$ premiers facteurs (resp. les $n-i$ derniers facteurs) de $L_{1}^{\otimes n}$. 
4.1. Noyau de $g_{n}: L_{1}^{\otimes n} \rightarrow J\left(2 p^{n}-2\right)$. - Comme la restriction du morphisme $\pi_{0}: L_{1} \rightarrow J(2 p-2)$ sur $L_{1,3}=y^{p-1} L_{1}$ est triviale, il est clair que $\mathfrak{M}_{n}=$ $L_{1}^{\otimes n-1} \otimes L_{1,3}$ est contenu dans le noyau de $g_{n}$.

D'autre part, on observe que si $X_{1}^{e} Y_{1}^{a} X_{2}^{f} Y_{2}^{b}$ est de degré $2(p-1)\left(p^{m}+p^{m-1}\right)$, alors $e=f=0$ et $a+b=p^{m}+p^{m-1}$. Comme $L_{2}=e_{2} \cdot\left(L_{1} \otimes L_{1}\right)$, le module $\mathfrak{M}_{i}$, $1 \leq i \leq n-1$, est également inclu dans le noyau de $g_{n}$ grâce à la proposition suivante.

Proposition 4.3. - Si $a+b=p^{m}+p^{m-1}$ et $a, b>0$, alors l'expression de $e_{2} \cdot Y_{1}^{a} Y_{2}^{b}$ comme somme de monômes distincts ne contient pas $Y_{1}^{p^{m}} Y_{2}^{p^{m-1}}$.

On a besoin du lemme suivant :

Lemme 4.4. - Supposons $a+b=p^{m}+p^{m-1}, a, b>0$ et $a, b \notin\left\{p^{m}, p^{m-1}\right\}$. Considérons les développements $p$-adiques de $(p-1) a$ et $(p-1) b$ :

$$
(p-1) a=\sum_{i=0}^{m} a_{i} p^{i}, \quad(p-1) b=\sum_{i=0}^{m} b_{i} p^{i}, \quad 0 \leq a_{i}, b_{i} \leq p-1 .
$$

Alors $a_{m-1}=p-1$ si et seulement si $b_{m-1}=p-1$.

Démonstration. - Supposons que $a_{m-1}=p-1$. On observe d'abord que

$$
0<\sum_{i=0}^{m-2} a_{i} p^{i}<p^{m-1} .
$$

En effet, si $\sum_{i=0}^{m-2} a_{i} p^{i}=0$, alors $(p-1) a=(p-1) p^{m-1}+a_{m} p^{m}$ ce qui implique soit $a_{m-1}=0$ soit $a_{m-1}=p-1$. Comme $a \neq p^{m-1}$, on a $a_{m-1}=p-1$, donc $a=p^{m}+p^{m-1}$ et $b=0$ ce qui n'est pas possible. L'inégalité $\sum_{i=0}^{m-2} a_{i} p^{i}<p^{m-1}$ est évidente.

On montre maintenant que $b_{m-1}=p-1$. On a

$$
(p-1) b=(p-1)\left(p^{m}+p^{m-1}-a\right)=\left(p-1-a_{m}\right) p^{m}-\sum_{i=0}^{m-2} a_{i} p^{i} .
$$

On en déduit que $\left(p-1-a_{m}\right) p^{m}-p^{m-1}<(p-1) b<\left(p-1-a_{m}\right) p^{m}$. On obtient donc

$$
b_{m}=\left[\frac{(p-1) b}{p^{m}}\right]=p-2-a_{m},
$$

$[x]$ désignant la partie entière de $x$. Il suit que

$$
(p-1) b-b_{m} p^{m}=\left(p-1-a_{m}-b_{m}\right) p^{m}-\sum_{i=0}^{m-2} a_{i} p^{i}=p^{m}-\sum_{i=0}^{m-2} a_{i} p^{i} .
$$


Il s'ensuit $p^{m}-p^{m-1}<(p-1) b-b_{m} p^{m}<p^{m}$ ce qui entraîne que

$$
b_{m-1}=\left[\frac{(p-1) b-b_{m} p^{m}}{p^{m-1}}\right]=p-1 \text {. }
$$

De manière analogue, on montre que si $b_{m-1}=p-1$ alors $a_{m-1}=p-1$. Le lemme est démontré.

Démonstration de la proposition 4.3. - On a

$$
e_{2} \cdot Y_{1}^{a} Y_{2}^{b}=\sum_{\lambda \in \mathbb{F}_{p}} y_{1}^{(p-1) a}\left(\lambda y_{1}+y_{2}\right)^{(p-1) b}-\sum_{\lambda \in \mathbb{F}_{p}} y_{1}^{(p-1) b}\left(\lambda y_{1}+y_{2}\right)^{(p-1) a}
$$

On note $c(a, b, m)$ le coefficient de $Y_{1}^{p^{m}} Y_{2}^{p^{m-1}}$ dans cette somme. On va montrer que $c(a, b, m)=0$ en considérant les cas suivants :

Cas $a>p^{m}$ et $b<p^{m-1}$. - On a

$$
c(a, b, m)=-\sum_{\lambda \in \mathbb{F}_{p}} \lambda^{(p-1)\left(p^{m}-b\right)}\left(\begin{array}{c}
(p-1) a \\
(p-1)\left(p^{m}-b\right)
\end{array}\right)=\left(\begin{array}{c}
(p-1) a \\
(p-1) p^{m-1}
\end{array}\right) .
$$

Ce coefficient est nul car dans le cas contraire, on a $a_{m-1}=p-1$, alors d'après le lemme $4.4, b_{m-1}=p-1$ et encore $(p-1) b \geq(p-1) p^{m-1}$, soit $b \geq p^{m-1}$ : absurde!

Cas $a=p^{m}$ et $b=p^{m-1}$. - On a

$$
c(a, b, m)=\sum_{\lambda \in \mathbb{F}_{p}} 1-\sum_{\lambda \in \mathbb{F}_{p}} \lambda^{(p-1)\left(p^{m}-b\right)}\left(\begin{array}{c}
(p-1) a \\
(p-1)\left(p^{m}-b\right)
\end{array}\right)=\left(\begin{array}{c}
(p-1) p^{m} \\
(p-1) p^{m-1}
\end{array}\right)=0 .
$$

Cas $p^{m-1}<a<p^{m}$ et $p^{m-1}<b<p^{m}$. - On a

$$
\begin{aligned}
c(a, b, m)= & \sum_{\lambda \in \mathbb{F}_{p}} \lambda^{(p-1)\left(p^{m}-a\right)}\left(\begin{array}{c}
(p-1) b \\
(p-1)\left(p^{m}-a\right)
\end{array}\right) \\
& -\sum_{\lambda \in \mathbb{F}_{p}} \lambda^{(p-1)\left(p^{m}-b\right)}\left(\begin{array}{c}
(p-1) a \\
(p-1)\left(p^{m}-b\right)
\end{array}\right) \\
= & -\left(\begin{array}{c}
(p-1) b \\
(p-1) p^{m-1}
\end{array}\right)+\left(\begin{array}{c}
(p-1) a \\
(p-1) p^{m-1}
\end{array}\right) .
\end{aligned}
$$

Le lemme 4.4 implique $c(a, b, m)=0$.

Cas $a=p^{m-1}$ et $b=p^{m}$. - On a

$c(a, b, m)=\sum_{\lambda \in \mathbb{F}_{p}} \lambda^{(p-1)\left(p^{m}-a\right)}\left(\begin{array}{c}(p-1) b \\ (p-1)\left(p^{m}-a\right)\end{array}\right)-\sum_{\lambda \in \mathbb{F}_{p}} 1=\left(\begin{array}{c}(p-1) p^{m} \\ (p-1) p^{m-1}\end{array}\right)=0$.

TOME $140-2012-\mathrm{N}^{\mathrm{O}} 2$ 
Cas $a<p^{m-1}$ et $b>p^{m}$. - On a

$$
c(a, b, m)=\sum_{\lambda \in \mathbb{F}_{p}} \lambda^{(p-1)\left(p^{m}-a\right)}\left(\begin{array}{c}
(p-1) b \\
(p-1)\left(p^{m}-a\right)
\end{array}\right)=-\left(\begin{array}{c}
(p-1) b \\
(p-1) p^{m-1}
\end{array}\right) .
$$

Comme dans le premier cas, ce coefficient est nul car dans le cas contraire, on a une contradiction : $a \geq p^{m-1}$. La proposition est démontrée.

4.2. Surjectivité de $g_{n}: L_{1}^{\otimes n} \rightarrow J\left(2 p^{n}-2\right)$. - Considérons la somme directe $\bar{J}(*)^{*}:=\bigoplus_{d \geq 0} J(2 d)$. Il découle de la description de H. Miller ([22]) de la somme directe de tous les modules de Brown-Gitler que

$$
\bar{J}(*)^{*} \cong \Lambda\left(\hat{x}_{j} \mid j \geq 0\right) \otimes \mathbb{F}_{p}\left[\hat{y}_{j} \mid j \geq 0\right]
$$

avec $\hat{x}_{j} \in J\left(2 p^{j}\right)^{1}$ et $\hat{y}_{j} \in J\left(2 p^{j}\right)^{2}$. L'action de l'algèbre de Steenrod est donnée par

$$
\beta \hat{x}_{j}=\hat{y}_{j}, \quad P^{1} \hat{y}_{j}=\hat{y}_{j-1}^{p}, \quad \hat{y}_{-1}=0
$$

et la formule de Cartan. Le bidegré de $\hat{x}_{j}$ est $\left(1,2 p^{j}\right)$ et le bidegré de $\hat{y}_{j}$ est $\left(2,2 p^{j}\right)$. Le module $J(2 d)$ est alors le sous-espace vectoriel ayant comme base les monômes de second degré $2 d$. En particulier, on a

Proposition 4.5. - Le module de Brown-Gitler $J\left(2 p^{n}-2\right)$ a une base formée par les éléments $\hat{x}_{0}^{\epsilon_{0}} \hat{y}_{0}^{i_{0}} \cdots \hat{x}_{n-1}^{\epsilon_{n-1}} \hat{y}_{n-1}^{i_{n-1}}$ avec $\sum_{j=0}^{n-1}\left(\epsilon_{j}+i_{j}\right) p^{j}=p^{n}-1, \epsilon_{j} \in\{0,1\}$, $i_{j} \geq 0$.

On considère ensuite la $\mathbb{F}_{p}$-algèbre $\Lambda\left(\tilde{x}_{0}, \cdots, \tilde{x}_{n-1}\right) \otimes \mathbb{F}_{p}\left[\tilde{y}_{0}, \cdots, \tilde{y}_{n-1}\right]$ avec $\left|\tilde{x}_{j}\right|=1$ et $\left|\tilde{y}_{j}\right|=2$, munie de l'action tordue de l'algèbre de Steenrod définie par

$$
\beta \tilde{x}_{j}=\tilde{y}_{j}, \quad P^{1} \tilde{y}_{i}=\tilde{y}_{i-1}^{p}, \quad \tilde{y}_{-1}=\tilde{y}_{n-1}
$$

et la formule de Cartan. Le résultat suivant est un remarquable théorème de rigidité concernant la structure de module instable de $H^{*} V_{n}$.

THÉORÈme $4.6([4])$. - Le module instable $\Lambda\left(\tilde{x}_{0}, \cdots, \tilde{x}_{n-1}\right) \otimes \mathbb{F}_{p}\left[\tilde{y}_{0}, \cdots, \tilde{y}_{n-1}\right]$ est isomorphe $\grave{a} H^{*} V_{n}$.

Remarque 4.7. - L'isomorphisme de Campbell-Selick

$$
\Lambda\left(x_{0}, \ldots, x_{n-1}\right) \otimes \mathbb{F}_{p}\left[y_{0}, \ldots, y_{n-1}\right] \cong \Lambda\left(\tilde{x}_{0}, \cdots, \tilde{x}_{n-1}\right) \otimes \mathbb{F}_{p}\left[\tilde{y}_{0}, \cdots, \tilde{y}_{n-1}\right]
$$

préserve respectivement la partie polynomiale et la partie extérieure. 
Pour tout monôme $\tilde{m}=\tilde{x}_{0}^{\epsilon_{0}} \tilde{y}_{0}^{i_{0}} \cdots \tilde{x}_{n-1}^{\epsilon_{n-1}} \tilde{y}_{n-1}^{i_{n-1}}$, le poids de $\tilde{m}$ est défini par

$$
w(\tilde{m}):=\sum_{j=0}^{n-1}\left(\epsilon_{j}+i_{j}\right) p^{j} .
$$

On note $\mathbf{C S}_{n}(k)$ le sous-espace de $\Lambda\left(\tilde{x}_{0}, \cdots, \tilde{x}_{n-1}\right) \otimes \mathbb{F}_{p}\left[\tilde{y}_{0}, \cdots, \tilde{y}_{n-1}\right]$ ayant pour base les monômes dont le poids est congru modulo $p^{n}-1$ à $k$. L'action tordue de l'algèbre de Steenrod sur $E\left(\tilde{x}_{0}, \cdots, \tilde{x}_{n-1}\right) \otimes \mathbb{F}_{p}\left[\tilde{y}_{0}, \cdots, \tilde{y}_{n-1}\right]$ montre que $\mathbf{C S}_{n}(k)$ est un $\mathscr{G}_{p}$-module. On obtient ainsi la décomposition de CampbellSelick de $H^{*} V_{n}$ en somme directe de $\mathscr{C}_{p}$-modules instables :

$$
H^{*} V_{n} \cong \Lambda\left(\tilde{x}_{0}, \cdots, \tilde{x}_{n-1}\right) \otimes \mathbb{F}_{p}\left[\tilde{y}_{0}, \cdots, \tilde{y}_{n-1}\right]=\bigoplus_{k=0}^{p^{n}-2} \mathbf{C S}_{n}(k)
$$

Proposition 4.8. - Le facteur $\mathbf{C S}_{n}(0)$ dans cette décomposition a une base formée par les monômes $\tilde{x}_{0}^{\epsilon_{0}} \tilde{y}_{0}^{i_{0}} \cdots \tilde{x}_{n-1}^{\epsilon_{n-1}} \tilde{y}_{n-1}^{i_{n-1}}$ qui vérifient $\sum_{j=0}^{n-1}\left(\epsilon_{j}+i_{j}\right) p^{j} \equiv 0$ $\bmod p^{n}-1$.

On obtient donc une surjection d'espaces vectoriels gradués :

$$
\overline{c s}_{n}: \mathbf{C S}_{n}(0) \rightarrow J\left(2 p^{n}-2\right)
$$

qui envoie $\tilde{m}:=\tilde{x}_{0}^{\epsilon_{0}} \tilde{y}_{0}^{i_{0}} \cdots \tilde{x}_{n-1}^{\epsilon_{n-1}} \tilde{y}_{n-1}^{i_{n-1}}$ sur $\hat{m}:=\hat{x}_{0}^{\epsilon_{0}} \hat{y}_{0}^{i_{0}} \cdots \hat{x}_{n-1}^{\epsilon_{n-1}} \hat{y}_{n-1}^{i_{n-1}}$ si $w(\tilde{m})=$ $p^{n}-1$ et sur 0 sinon.

Proposition 4.9. - La surjection $\overline{c s}_{n}$ est $\mathscr{G}_{p}$-linéaire. Elle envoie $\tilde{y}_{0}^{p^{n}-1}$ sur la classe canonique $\iota_{2 p^{n}-2}$ de $J\left(2 p^{n}-2\right)$ et envoie les autres monômes de degré $2 p^{n}-2$ sur 0 .

Démonstration. - Comme $\beta\left(\tilde{x}_{j}\right)=\tilde{y}_{j}$ pour tout $j$, on a $w(\beta(\tilde{m}))=w(\tilde{m})$. La $\beta$-linéarité de $\overline{c s}_{n}$ est alors évidente puisque $\beta\left(\hat{x}_{j}\right)=\hat{y}_{j}$. Il reste à vérifier que $\overline{c s}_{n}$ est $P^{1}$-linéaire. On a

$$
\begin{aligned}
& P^{1}(\tilde{m})=\tilde{x}_{0}^{\epsilon_{0}} \tilde{y}_{0}^{i_{0}} P^{1}\left(\tilde{x}_{1}^{\epsilon_{1}} \tilde{y}_{1}^{i_{1}} \cdots \hat{x}_{n-1}^{\epsilon_{n-1}} \hat{y}_{n-1}^{i_{n-1}}\right)+\frac{i_{0} \hat{y}_{n-1}^{p}}{\hat{y}_{0}} \tilde{m} \\
& P^{1}(\hat{m})=\hat{x}_{0}^{\epsilon_{0}} \hat{y}_{0}^{i_{0}} P^{1}\left(\hat{x}_{1}^{\epsilon_{1}} \hat{y}_{1}^{i_{1}} \cdots \hat{x}_{n-1}^{\epsilon_{n-1}} \hat{y}_{n-1}^{i_{n-1}}\right) .
\end{aligned}
$$

Comme $w\left(\frac{\hat{y}_{n-1}^{p}}{\hat{y}_{0}} \tilde{m}\right)=w(\tilde{m})+p^{n}-1$, on a $\overline{c s}_{n}\left(\frac{\hat{y}_{n-1}^{p}}{\hat{y}_{0}} \tilde{m}\right)=0$ si $w(\tilde{m})=p^{n}-1$. La $P_{1}$-linéarité de $\overline{c s}_{n}$ suit. 
Le deuxième énoncé de la proposition provient de ce que le système d'équations

$$
\begin{aligned}
& \left(\epsilon_{0}+i_{0}\right)+\sum_{j=1}^{n-1}\left(\epsilon_{j}+i_{j}\right) p^{j}=p^{n}-1 \\
& \left(\epsilon_{0}+2 i_{0}\right)+\sum_{j=1}^{n-1}\left(\epsilon_{j}+2 i_{j}\right)=2\left(p^{n}-1\right)
\end{aligned}
$$

admet pour solution unique $: i_{0}=p^{n}-1, i_{2}=\cdots=i_{n-1}=\epsilon_{0}=\cdots=\epsilon_{n-1}=0$. En effet, la première équation implique d'une part que $i_{0} \leq p^{n}-1$. D'autre part, en soustrayant la première équation de la deuxième, on a

$$
i_{0}-\sum_{j=1}^{n-1}\left[\left(\epsilon_{j}+i_{j}\right) p^{j}-\left(\epsilon_{j}+2 i_{j}\right)\right]=p^{n}-1,
$$

d'où $i_{0} \geq p^{n}-1$. Ainsi $i_{0}=p^{n}-1$ et $i_{2}=\cdots=i_{n-1}=\epsilon_{0}=\cdots=\epsilon_{n-1}=0$.

On obtient de ce qui précède une surjection de modules instables :

$$
c s_{n}: H^{*} V_{n} \rightarrow C S_{n}(0) \stackrel{\overline{c s}_{n}}{\longrightarrow} J\left(2 p^{n}-2\right) .
$$

On considère ensuite le morphisme

$$
\tilde{g}_{n}: H^{*} V_{n} \rightarrow J\left(2 p^{n}-2\right)
$$

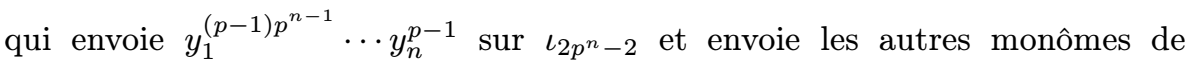
$H^{2 p^{n}-2} V_{n}$ sur 0 . Autrement dit, si $\left\{a_{1}, \cdots, a_{n}\right\}$ (resp. $\left\{b_{1}, \cdots, b_{n}\right\}$ ) est la base de $V_{n}=H_{1} V_{n}$ (resp. $\left.W_{n} \subset H_{2} V_{n}\right)$ qui est duale à la base $\left\{x_{1}, \cdots, x_{n}\right\}$ (resp. $\left.\left\{y_{1}, \cdots, y_{n}\right\}\right)$ de $V_{n}^{*}=H^{1} V_{n}$ (resp. $\left.W_{n}^{*}=\beta H^{1} V_{n}\right)$, le morphisme $\tilde{g}_{n}$ est alors représenté par le monôme $b_{1}^{\left((p-1) p^{n-1}\right)} \cdots b_{n}^{(p-1)}$ dans la puissance divisée $\Gamma^{p^{n}-1}\left(W_{n}\right)$.

Comme l'isomorphisme de Campbell-Selick

$$
H^{*} V_{n} \cong \Lambda\left(\tilde{x}_{0}, \cdots, \tilde{x}_{n-1}\right) \otimes \mathbb{F}_{p}\left[\tilde{y}_{0}, \cdots, \tilde{y}_{n-1}\right]
$$

préserve respectivement la partie polynomiale et la partie extérieure, le morphisme $c s_{n}$ ci-dessus est aussi représenté par un élément, noté $\mathbf{c s}_{n}$, de la puissance divisée $\Gamma^{p^{n}-1}\left(W_{n}\right)$.

On a le diagramme suivant dont le triangle en bas est commutatif et la flèche verticale à droite est la projection naturelle :

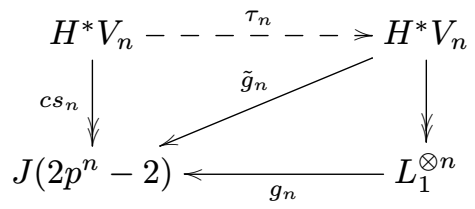


Proposition 4.10. - Il existe un endomorphisme $\tau_{n}$ de $H^{*} V_{n}$ qui rend commutatif le diagramme ci-dessus.

Corollaire 4.11. - Le morphisme $g_{n}$ est surjectif.

Pour montrer l'existence du morphisme $\tau_{n}$, il suffit de montrer qu'il existe un élément de $\mathbb{F}_{p}\left[\operatorname{Mat}\left(\mathbb{F}_{p}\right)\right]$ qui envoie $b_{1}^{\left((p-1) p^{n-1}\right)} \cdots b_{n}^{(p-1)}$ sur $\mathbf{c s}_{n}$. Ceci résulte du résultat général suivant dont la démonstration est renvoyée à la section 4.4.

THÉORÈme 4.12. - En tant que $\mathbb{F}_{p}\left[\operatorname{Mat}\left(\mathbb{F}_{p}\right)\right]$-module, $\Gamma^{p^{n}-1}\left(W_{n}\right)$ est engendré $\operatorname{par} b_{1}^{\left((p-1) p^{n-1}\right)} \cdots b_{n}^{(p-1)}$.

4.3. Base acceptable de $J\left(2 p^{n}-2\right)$. - Dans cette section on construit une base de $J\left(2 p^{n}-2\right)$.

Proposition 4.13. - Le quotient $L_{1}^{\otimes n} / \sum_{i=1}^{n} \mathfrak{M}_{i}$ est engendré par les monômes $X_{1}^{\epsilon_{1}} Y_{1}^{i_{1}} \cdots X_{n}^{\epsilon_{n}} Y_{n}^{i_{n}}$ qui vérifient $\epsilon_{j} \in\{0,1\}, 1 \leq i_{j} \leq p i_{j+1}-\epsilon_{j+1}$ et $i_{n}=1$.

Démonstration. - On appelle acceptable tout monôme $\omega_{\left(1^{n}\right)}^{I}:=$ $X_{1}^{\epsilon_{1}} Y_{1}^{i_{1}} \cdots X_{n}^{\epsilon_{n}} Y_{n}^{i_{n}}$ (voir les notations 2.10 et 3.4 ) qui vérifie $\epsilon_{j} \in\{0,1\}$, $1 \leq i_{j} \leq p i_{j+1}-\epsilon_{j+1}$ et $i_{n}=1$. Supposons $\omega_{\left(1^{n}\right)}^{I}$ n'est pas acceptable.

- Si $i_{n}>1$ alors $\omega_{\left(1^{n}\right)}^{I}=0$ dans le quotient puisque $\omega_{\left(1^{n}\right)}^{I} \in \mathfrak{M}_{n}$.

- Si $i_{j}>p i_{j+1}-\epsilon_{j+1}$ pour $1 \leq j<n$, d'après le lemme 2.11, on a $\omega_{\left(1^{j-1}, 2,1^{n-j-1}\right)}^{I}=\omega_{\left(1^{n}\right)}^{I}+\operatorname{des}\left(1^{n}\right)$-monômes d'ordre supérieur et $\omega_{\left(1^{j-1}, 2,1^{n-j-1}\right)}^{I}=0$ dans le quotient $\operatorname{car} \omega_{\left(1^{j-1}, 2,1^{n-j-1}\right)}^{I} \in \mathfrak{M}_{j}$.

Une itération évidente donne le résultat.

REMARQUE 4.14. - L'argument ci-dessus permet aussi de conclure que le module quotient

$$
J_{n, m}:=\frac{L_{1}^{\otimes n}}{\sum_{i=1}^{n-1} L_{1}^{\otimes i-1} \otimes L_{2} \otimes L_{1}^{\otimes n-i-1}+L_{1}^{\otimes n-1} \otimes L_{1, m}}
$$

est engendré par les classes des monômes $X_{1}^{\epsilon_{1}} Y_{1}^{i_{1}} \cdots X_{n}^{\epsilon_{n}} Y_{n}^{i_{n}}$ qui vérifient $\epsilon_{j} \in$ $\{0,1\}, 1 \leq i_{j} \leq p i_{j+1}-\epsilon_{j+1}$ et $1 \leq i_{n} \leq \frac{m-1}{2}$. En particulier, $J_{n, m}$ est un module fini.

TOME $140-2012-\mathrm{N}^{\mathrm{O}} 2$ 
Considérons ensuite les deux ensembles suivants :

$$
\begin{aligned}
& \mathscr{L}(n):=\left\{\left(\epsilon_{1}, i_{1} ; \cdots ; \epsilon_{n}, i_{n}\right) \mid \epsilon_{k} \in\{0,1\}, 1 \leq i_{k} \leq p i_{k+1}-\epsilon_{k+1}, i_{n}=1\right\}, \\
& \mathcal{J}(n):=\left\{\left(\epsilon_{0}, i_{0} ; \cdots ; \epsilon_{n-1}, i_{n-1}\right) \mid \sum_{j=0}^{n-1}\left(\epsilon_{j}+i_{j}\right) p^{j}=p^{n}-1\right\} .
\end{aligned}
$$

On définit respectivement le degré de $\left(\epsilon_{1}, i_{1} ; \cdots ; \epsilon_{n}, i_{n}\right) \in \mathcal{L}(n)$ comme étant le degré de $X_{1}^{\epsilon_{1}} Y_{1}^{i_{1}} \cdots X_{n}^{\epsilon_{n}} Y_{n}^{i_{n}}$, soit $\sum_{k=1}^{n} 2(p-1) i_{k}-\epsilon_{k}$, et le degré de $\left(\epsilon_{0}, i_{0} ; \cdots ; \epsilon_{n-1}, i_{n-1}\right) \in \mathcal{J}(n)$ comme étant le premier degré de $\hat{x}_{0}^{\epsilon_{0}} \hat{y}_{0}^{i_{0}} \cdots \hat{x}_{n-1}^{\epsilon_{n-1}} \hat{y}_{n-1}^{i_{n-1}}$, soit $\sum_{k=0}^{n-1} 2 i_{k}+\epsilon_{k}$.

On vérifie sans peine que les deux correspondances suivantes

$$
\begin{aligned}
\mathscr{L}(n) & \longrightarrow \mathcal{J}(n) \\
\left(\epsilon_{j}, i_{j}\right)_{1 \leq j \leq n} & \longmapsto\left(\epsilon_{j+1}, p i_{j+1}-\epsilon_{j+1}-i_{j}\right)_{0 \leq j \leq n-1}, \quad\left(i_{0}=1\right)
\end{aligned}
$$

et

$$
\begin{aligned}
\mathscr{J}(n) & \longrightarrow \mathscr{L}(n) \\
\left(\epsilon_{j}, i_{j}\right)_{0 \leq j \leq n-1} & \longmapsto\left(\epsilon_{j+1}, \frac{1+\sum_{k=0}^{j}\left(\epsilon_{k+1}+i_{k}\right) p^{k}}{p^{j}}\right)_{0 \leq j \leq n-1}
\end{aligned}
$$

sont bien définies et inverses l'une de l'autre. De plus, elles préservent le degré. On en déduit que, en chaque degré, la dimension du module quotient $L_{1}^{\otimes n} / \sum_{i=1}^{n} \mathfrak{M}_{i}$ est inférieure ou égale à celle du module de Brown-Gitler $J\left(2 p^{n}-2\right)$. La surjectivité de $g_{n}$ est utilisée pour obtenir la

\section{Proposition 4.15. - Les éléments}

$$
g_{n}\left(X_{1}^{\epsilon_{1}} Y_{1}^{i_{1}} \cdots X_{n}^{\epsilon_{n}} Y_{n}^{i_{n}}\right), \quad\left(\epsilon_{1}, i_{1} ; \cdots ; \epsilon_{n}, i_{n}\right) \in \mathcal{L}(n),
$$

forment une base de $J\left(2 p^{n}-2\right)$.

4.4. $\Gamma^{p^{n}-1}\left(W_{n}\right)$ en tant que $\mathbb{F}_{p}\left[\operatorname{Mat}_{n}\left(\mathbb{F}_{p}\right)\right]$-module. - Dans cette section, on montre que le $\mathbb{F}_{p}\left[\operatorname{Mat}_{n}\left(\mathbb{F}_{p}\right)\right]$-module $\Gamma^{p^{n}-1}\left(W_{n}\right)$ est engendré par $b_{1}^{\left((p-1) p^{n-1}\right)} \cdots b_{n}^{(p-1)}$. Cela est équivalent à démontrer la surjectivité du morphisme suivant :

$$
\mathbb{F}_{p}\left[\operatorname{Mat}_{n}\left(\mathbb{F}_{p}\right)\right] \rightarrow \Gamma^{p^{n}-1}\left(W_{n}\right), \quad \sigma \mapsto\left(b_{1}^{\left((p-1) p^{n-1}\right)} \cdots b_{n}^{(p-1)}\right) \cdot \sigma .
$$

On se ramène alors à démontrer l'injectivité du morphisme dual :

$$
S^{p^{n}-1}\left(W_{n}^{*}\right) \rightarrow \mathbb{F}_{p}^{\operatorname{Mat}_{n}\left(\mathbb{F}_{p}\right)}, \quad P \mapsto\left(\sigma \rightarrow\left\langle b_{1}^{\left((p-1) p^{n-1}\right)} \cdots b_{n}^{(p-1)}, \sigma \cdot P\right\rangle\right) .
$$

Ici $\langle$,$\rangle désigne l'accouplement canonique entre \Gamma^{p^{n}-1}\left(W_{n}\right)$ et $S^{p^{n}-1}\left(W_{n}^{*}\right)$. On rappelle que $\langle B \cdot \sigma, P\rangle=\langle B, \sigma \cdot P\rangle$ pour tout $\sigma \in \operatorname{Mat}_{n}\left(\mathbb{F}_{p}\right)$, tout $B \in \Gamma^{p^{n}-1}\left(W_{n}\right)$ et tout $P \in S^{p^{n}-1}\left(W_{n}^{*}\right)$. 
On a ainsi vérifié que le théorème 4.12 est équivalent à la proposition suivante.

Proposition 4.16. - Pour tout $P \in S^{p^{n}-1}\left(W_{n}^{*}\right)$, il existe $\sigma \in \operatorname{Mat}_{n}\left(\mathbb{F}_{p}\right)$ telle que l'expression de $\sigma \cdot P$ comme somme de monômes distincts contient le monôme $y_{1}^{(p-1) p^{n-1}} \cdots y_{n}^{p-1}$.

Pour toute suite d'entiers $I=\left(i_{1}, \ldots, i_{n}\right)$, notons $y^{I}=y_{1}^{i_{1}} \cdots y_{n}^{i_{n}}$. Appelons admissible tout monôme $y^{I}$ qui vérifie $i_{s-1} \geq p i_{s}$ pour $1<s \leq n$. Mettons l'ordre lexicographique sur les monômes de $S^{*}\left(W_{n}^{*}\right)$. Pour tout élément homogène non-nul $P$ de $S^{*}\left(W_{n}^{*}\right)$, soit $\mu(P)$ le plus grand monôme (par rapport à l'ordre lexicographique) qui apparaît dans $P$.

Lemme 4.17. - Si $\mu(P)$ n'est pas admissible, il existe $\sigma \in \operatorname{Mat}_{n}\left(\mathbb{F}_{p}\right)$ tel que $\mu(\sigma \cdot P)>\mu(P)$.

Démonstration. - Soit $\mu(P)=y_{1}^{i_{1}} \cdots y_{n}^{i_{n}}$. Comme $\mu(P)$ n'est pas admissible, il existe $1<s \leq n$ tel que $i_{s-1}<p i_{s}$. En regroupant les monômes de $P$, on le réécrit sous la forme $P=y_{1}^{i_{1}} \cdots y_{s-2}^{i_{s-2}} Q y_{s+1}^{i_{s+1}} \cdots y_{n}^{i_{n}}+R$, de manière que $\mu(R)<$ $\mu(P)$ et que $Q=Q\left(y_{s-1}, y_{s}\right) \in \mathbb{F}_{p}\left[y_{s-1}, y_{s}\right]$ est un polynôme de degré $i_{s-1}+i_{s}$. Observons que pour tout $\sigma \in \operatorname{Mat}_{n}\left(\mathbb{F}_{p}\right)$ correspondant aux substitutions qui ne font intervenir que $y_{s-1}, y_{s}$, les monômes de $\sigma \cdot R$ sont différents des monômes de $y_{1}^{i_{1}} \cdots y_{s-2}^{i_{s-2}}(\sigma \cdot Q) y_{s+1}^{i_{s+1}} \cdots y_{n}^{i_{n}}$. Par conséquent, si un tel $\sigma$ vérifie $\mu(\sigma \cdot Q)>$ $y_{s-1}^{i_{s-1}} y_{s}^{i_{s}}$, on aura forcément $\mu(\sigma \cdot P)>\mu(P)$ (car $\mu(\sigma \cdot P)$ est supérieur ou égal à $\mu\left(y_{1}^{i_{1}} \cdots y_{s-2}^{i_{s-2}}(\sigma \cdot Q) y_{s+1}^{i_{s+1}} \cdots y_{n}^{i_{n}}\right)$ qui est strictement supérieur à $\left.\mu(P)\right)$.

On a ainsi montré qu'il suffit de vérifier le lemme pour $n=2$. Soit $\mu(P)=$ $y_{1}^{i_{1}} y_{2}^{i_{2}}$ avec $i_{1}<p i_{2}$. On pose $P=\left(y_{1} y_{2}^{p}-y_{1}^{p} y_{2}\right)^{q} Q$ avec $Q \in S^{i}\left(V_{2}^{*}\right)$ et l'entier $q$ le plus grand possible.

Cas 1. - $Q$ contient un monôme $y_{1}^{i}$. Celui-ci est forcément $\mu(Q)$, d'où $\mu(P)=$ $y_{1}^{p q+i} y_{2}^{q}$, ce qui est absurde puisque $\mu(P)=y_{1}^{i_{1}} y_{2}^{i_{2}}$ et $i_{1}<p i_{2}$.

Cas 2. - $Q$ contient un monôme $y_{2}^{i}$. Soit $\sigma \in \operatorname{Mat}_{2}\left(\mathbb{F}_{p}\right)$ qui échange $y_{1}$ et $y_{2}$. Alors

$$
\mu(\sigma \cdot P)=y_{1}^{p q+i} y_{2}^{q}
$$

Cas 3. $-Q$ est divisible par $y_{1} y_{2}$. Notons que $y_{1} y_{2}^{p}-y_{1}^{p} y_{2}=y_{1} y_{2} \prod_{a \in \mathbb{F}_{p}^{*}}\left(y_{2}-\right.$ $\left.a y_{1}\right)$. La maximalité de $q$ implique alors qu'il existe $a \in \mathbb{F}_{p}^{*}$ tel que $Q$ n'est pas divisible par $y_{2}-a y_{1}$. Soit $\sigma \in \operatorname{Mat}_{2}\left(\mathbb{F}_{p}\right)$ qui fixe $y_{1}$ et transforme $y_{2}$ en $a y_{1}+y_{2}$. Alors

$$
\sigma \cdot P=\left(y_{1} y_{2}^{p}-y_{1}^{p} y_{2}\right)^{q} Q\left(y_{1}, a y_{1}+y_{2}\right)
$$

TOME $140-2012-\mathrm{N}^{\mathrm{O}} 2$ 
Le terme qui ne comporte pas $y_{2}$ dans $Q\left(y_{1}, a y_{1}+y_{2}\right)$ est égal à $Q\left(y_{1}, a y_{1}\right)$, qui est non nul puisque $Q$ n'est pas divisible par $y_{2}-a y_{1}$. Supposons que $Q\left(y_{1}, a y_{1}\right)=b y_{1}^{i}$, alors

$$
\mu(\sigma \cdot P)=y_{1}^{p q+i} y_{2}^{q} .
$$

Observons maintenant que si $p q+i+q=i_{1}+i_{2}$, alors $(p q+i, q)>\left(i_{1}, i_{2}\right)$, car dans le cas contraire, on a $i_{1} \geq p q+i$, ce qui implique $q \geq i_{2}$, et alors $i_{1} \geq p i_{2}+i \geq p i_{2}$. Le lemme suit.

Démonstration de la proposition 4.16. - Posons $Y_{i}=y_{i}^{p-1}$. Montrons la proposition par récurrence sur $n$. Le cas $n=1$ est trivial. Supposons que $n>1$ et que la proposition soit vraie pour $n-1$. En posant $W_{n-1}^{*}=\mathbb{F}_{p}\left\{y_{2}, \cdots, y_{n}\right\}$, cette hypothèse implique que pour tout $Q \in S^{p^{n-1}-1}\left(W_{n-1}^{*}\right)$, il existe $\tau \in \operatorname{Mat}_{n}\left(\mathbb{F}_{p}\right)$ tel que $\tau \cdot Q$ contient $Y_{2}^{p^{n-2}} \cdots Y_{n-1}^{p} Y_{n}$. Ici $\tau$ ne fait intervenir que les générateurs $y_{2}, \ldots, y_{n}$.

Soit $P$ un élément quelconque non-nul de $S^{p^{n}-1}\left(W_{n}^{*}\right)$. Il faut montrer que $\sigma \cdot P$ contient $Y_{1}^{p^{n-1}} \cdots Y_{n-1}^{p} Y_{n}$ pour un certain $\sigma \in \operatorname{Mat}_{n}\left(\mathbb{F}_{p}\right)$. D'après le lemme 4.17, on peut supposer que $m(P)=y_{1}^{i_{1}} \cdots y_{n}^{i_{n}}$ est un monôme admissible. Mais alors $i_{1} \geq(p-1) p^{n-1}$. Réécrivons $P$ sous la forme $P=Y_{1}^{p^{n-1}} f+R$, où $f=f\left(y_{1}, \ldots, y_{n}\right)$ et $R$ ne contient que des monômes dont la puissance de $y_{1}$ est inférieure à $(p-1) p^{n-1}$.

Soit $u$ une combinaison linéaire non nulle des générateurs $y_{2}, \ldots, y_{n}$. Soit $\sigma_{u}$ la matrice définie par la substitution $y_{1}:=y_{1}+u$. On considère $\sigma_{u} \cdot P$ comme un élément de $\mathbb{F}_{p}\left[y_{2}, \cdots, y_{n}\right]\left[y_{1}\right]$. Il est clair que le coefficient de $Y_{1}^{p^{n-1}}$ dans $\sigma_{u} \cdot R$ est nul. Puisque $f$ est de degré $p^{n-1}-1$, on vérifie que le coefficient de $Y_{1}^{p^{n-1}}$ dans

$$
\sigma_{u} \cdot Y_{1}^{p^{n-1}} f\left(y_{1}, \cdots, y_{n}\right)=\sum_{i=0}^{p-1}(-1)^{i} y_{1}^{p^{n-1}(p-1-i)} u^{p^{n-1} i} f\left(y_{1}+u, y_{2}, \cdots, y_{n}\right)
$$

est $f\left(u, y_{2}, \ldots, y_{n}\right)$.

Cas 1. - Il existe $u$ telle que $f\left(u, y_{2}, \ldots, y_{n}\right) \neq 0$. D'après l'observation plus haut, il existe $\tau \in \operatorname{Mat}_{n}\left(\mathbb{F}_{p}\right)$ tel que $\tau \cdot f\left(u, y_{2}, \ldots, y_{n}\right)$ contient $Y_{2}^{p^{n-2}} \cdots Y_{n-1}^{p} Y_{n}$. Comme $\tau$ ne fait pas intervenir $y_{1}$, il suit que $\tau \sigma_{u} \cdot P$ contient $Y_{1}^{p^{n-1}} \cdots Y_{n-1}^{p} Y_{n}$.

Cas 2. - $f\left(u, y_{2}, \ldots, y_{n}\right)=0$ pour toute combinaison non nulle $u$. Ceci implique que le polynôme $f\left(y_{1}, \ldots, y_{n}\right)$ est divisible par $y_{1}+u$ pour toute $u$. Comme $f \in S^{p^{n-1}-1}\left(W_{n}^{*}\right)$, il suit que $f=a \prod_{0 \neq u \in V_{n-1}^{*}}\left(y_{1}+u\right)$ pour certain $a \in \mathbb{F}_{p}^{*}$. Clairement $\prod_{0 \neq u \in W_{n-1}^{*}}\left(y_{1}+u\right)$ contient $Y_{2}^{p^{n-2}} \cdots Y_{n-1}^{p} Y_{n}$. D'où $P$ lui-même contient $Y_{1}^{p^{n-1}} \cdots Y_{n-1}^{p} Y_{n}$. 
La proposition est démontrée.

\section{Application : cohomotopie du spectre $L(n, 3)$}

Dans cette section, on étudie la cohomotopie du spectre $L(n, 3)$ dans la catégorie des spectres $p$-complétés. On note $\left[L(n, 3), S^{k}\right]$ le groupe abélien des applications du spectre $L(n, 3)$ dans le spectre des suspensions itérées de la sphère $S^{k}$. On désigne par $\mathcal{M}$ la catégorie dont les objets sont les $\mathscr{G}_{p}$-modules gradués et dont les morphismes sont les applications $\mathscr{G}_{p}$-linéaires de degré zéro. La catégorie $\mathcal{U}$ des $\mathscr{A}_{p}$-modules instables est alors une sous catégorie pleine de $\mathcal{M}$.

Utilisant la suite spectrale d'Adams

$$
\operatorname{Ext}_{\mathcal{M}}^{s}\left(\Sigma^{-t} \mathbb{Z} / p, L_{n, 3}\right) \Longrightarrow\left[L(n, 3), S^{s-t}\right]
$$

on se ramène à l'étude des groupes d'extension $\operatorname{Ext}_{\mathcal{M}}^{s}\left(\Sigma^{-t} \mathbb{Z} / p, L_{n, 3}\right)$. A cette fin, on se servira de la suite spectrale de Grothendieck

$$
\operatorname{Ext}_{\mathcal{U}}^{u}\left(D_{v} \Sigma^{-t} \mathbb{Z} / p, L_{n, 3}\right) \Longrightarrow \operatorname{Ext}_{\mathcal{M}}^{u+v}\left(\Sigma^{-t} \mathbb{Z} / p, L_{n, 3}\right)
$$

pour passer de la catégorie $\mathcal{U}$ des $\mathscr{C}_{p}$-modules instables à celle des $\mathscr{C}_{p}$-modules à travers les dérivés $D_{v}$ du foncteur de déstabilisation (voir la section 5.1). La suite exacte du théorème 1.1 sera utilisée pour calculer les groupes d'extension $\operatorname{Ext}_{\mathcal{U}}^{u}\left(D_{v} \Sigma^{-t} \mathbb{Z} / p, L_{n, 3}\right)$. En particulier, on cherche à comprendre les groupes

$$
\operatorname{Hom}_{u}\left(D_{v} \Sigma^{-t} \mathbb{Z} / p, L_{n-u} \otimes J\left(2 p^{u}-2\right)\right),
$$

ou encore le module instable

$$
\left(D_{v} \Sigma^{-t} \mathbb{Z} / p: L_{n-u}\right) u
$$

la division dans $U$ de $D_{v} \Sigma^{-t} \mathbb{Z} / p$ par le module de Steinberg $L_{n-u}$. On se restreint à la division par $L_{1}$, le cas général est difficile et sera étudié ailleurs. En particulier, on explicitera une décomposition de $\left(D(n): L_{1}\right) u$ en termes des invariants modulaires de $\mathrm{GL}_{n}$. Ici $D(n)$ est l'image de la restriction $H^{*} \Sigma_{p^{n}} \rightarrow$ $H^{*}(\mathbb{Z} / p)^{n}$ induite par l'inclusion canonique $(\mathbb{Z} / p)^{n} \hookrightarrow \Sigma_{p^{n}}$. Pour $p$ impair, $D(n)$ est en général strictement inclu dans $\left(H^{*}(\mathbb{Z} / p)^{n}\right)^{\mathrm{GL}_{n}}$ : c'est là une différence fondamentale avec le cas $p=2$.

On exposera le calcul du groupe $\operatorname{Ext}_{\mathcal{M}}^{s}\left(\Sigma^{-t} \mathbb{Z} / p, L_{n, 3}\right)$ pour $s-t \geq 2 p^{n-2}+n$. Par conséquent, on obtiendra un résultat de type "conjecture de Segal" pour la cohomotopie du spectre $L(n, 3)$.

ThÉORÈme 5.1. - Supposons $k \geq 2 p^{n-2}+n$. Alors

$$
\left[L(n, 3), S^{k}\right]= \begin{cases}\mathbb{Z} / p & \text { si } k=2 p^{n}-2+n, \\ \mathbb{Z} / p & \text { si } k=2 p^{n-1}-1+n, \\ 0 & \text { sinon. }\end{cases}
$$

TOME $140-2012-\mathrm{N}^{\mathrm{O}} 2$ 
5.1. Dérivés du foncteur de déstabilisation. - On note $D: \mathcal{M} \rightarrow \mathcal{U}$ et on appelle foncteur de déstabilisation l'adjoint à gauche du foncteur oubli $\theta: \mathcal{U} \rightarrow \mathcal{M}$. Le foncteur $D$ est exact à droite et on note $D_{s}: \mathcal{M} \rightarrow \mathcal{U}, s \geq 0$, ses foncteurs dérivés.

On considère $\Sigma_{p^{n}}$ le groupe des permutations (de l'ensemble des points) du groupe $V_{n}=(\mathbb{Z} / p)^{n}$ et $i_{n}: V_{n} \hookrightarrow \Sigma_{p^{n}}$ le plongement via l'action par transla-

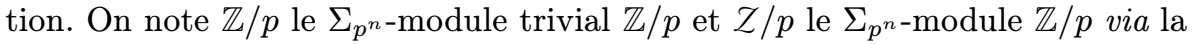
signature. On pose

$$
\begin{aligned}
& D(n)=\operatorname{Im}\left(H^{*}\left(\Sigma_{p^{n}} ; \mathbb{Z} / p\right) \stackrel{i_{n}^{*}}{\longrightarrow} H^{*}\left(V_{n} ; \mathbb{Z} / p\right)\right), \\
& \mathfrak{D}(n)=\operatorname{Im}\left(H^{*}\left(\Sigma_{p^{n}} ; Z / p\right) \stackrel{i_{n}^{*}}{\longrightarrow} H^{*}\left(V_{n} ; i_{n}^{*} Z / p\right)\right) .
\end{aligned}
$$

Comme le plongement naturel $i_{n}$ envoie $V_{n}$ dans le sous-groupe alterné $\mathscr{A}_{p^{n}}$ de $\Sigma_{p^{n}}, \mathcal{H}^{*} V_{n}:=H^{*}\left(V_{n} ; i_{n}^{*} Z / p\right)$ s'identifie à $H^{*} V_{n}:=H^{*}\left(V_{n} ; \mathbb{Z} / p\right)$. Néanmoins la structure de $\mathcal{H}^{*} V_{n}$ en tant que $\mathrm{GL}_{n}$-module est donnée par l'action tordue [24] :

$$
g * f:=\operatorname{det}(g)^{\frac{p-1}{2}}(g \cdot f)
$$

où $g \cdot f$ désigne l'action naturelle de $g \in \mathrm{GL}_{n}$ sur $f \in H^{*} V_{n}$. On observera que $D(n) \subset\left(H^{*} V_{n}\right)^{\mathrm{GL}_{n}}$ et $\mathfrak{D}(n) \subset\left(\mathcal{H}^{*} V_{n}\right)^{\mathrm{GL}_{n}}$.

On considère ensuite l'application $\mathbb{Z} / p$-linéaire

$$
\mathrm{St}_{n}\left(x_{1}, y_{1}, \ldots, x_{n}, y_{n} ;-\right): M \rightarrow H^{*} V_{n} \otimes M
$$

définie de manière récurrente comme suit ([24, 2.4.1]) :

$$
\begin{aligned}
\mathrm{St}_{1}\left(x_{1}, y_{1} ; m\right) & =\sum_{\substack{\epsilon=0,1 \\
0 \leq \epsilon+2 i \leq|m|}}(-1)^{\epsilon+i}\left(\frac{x_{1}}{y_{1}}\right)^{\epsilon} y_{1}^{\frac{(|m|-2 i)(p-1)}{2}} \otimes \beta^{\epsilon} P^{i}(m), \\
\mathrm{St}_{s}\left(x_{1}, y_{1}, \ldots, x_{s}, y_{s} ; m\right) & =\mathrm{St}_{1}\left(x_{1}, y_{1} ; \mathrm{St}_{s-1}\left(x_{2}, y_{2}, \ldots, x_{s}, y_{s} ; m\right)\right) .
\end{aligned}
$$

On abrègera la notation $\operatorname{St}_{n}\left(x_{1}, y_{1}, \ldots, x_{n}, y_{n} ; m\right)$ en $\operatorname{St}_{n}(m)$.

Soit $M$ un module instable. Le module instable $R_{n} M$ est défini par

$$
R_{n} M=D(n) \cdot \mathrm{St}_{n} M^{+} \oplus \mathfrak{D}(n) \cdot \mathrm{St}_{n} M^{-},
$$

où $M^{+}$(resp. $M^{-}$) désigne le sous-espace de $M$ formé des éléments de degré pair (resp. impair).

ThÉORÈme 5.2 ([24]). — Soit $M$ un module instable. Alors, pour tout $s \geq 0$, on a un isomorphisme de modules instables $D_{s} \Sigma^{1-s} M \cong \Sigma R_{s} M$. 
Rappelons que, à signe près, la classe d'Euler de la représentation régulière réelle réduite de $V_{n}$ est donnée par

$$
\mathfrak{e}_{n}=\left|\begin{array}{ccc}
y_{1} & \cdots & y_{n} \\
y_{1}^{p} & \cdots & y_{n}^{p} \\
\vdots & \cdots & \vdots \\
y_{1}^{p^{s-1}} & \cdots & y_{n}^{p^{s-1}}
\end{array}\right|^{\frac{p-1}{2}}
$$

La formule suivante sera utile pour le calcul des groupes $\operatorname{Ext}_{\mathcal{M}}^{s}\left(\Sigma^{-t} \mathbb{Z} / p, L_{n, m}\right)$.

Corollaire 5.3. - Supposons $s \geq 0$ et $s+t \geq 1$. Alors

$$
D_{s} \Sigma^{t} \mathbb{Z} / p \cong \begin{cases}\Sigma^{s+t} D(s) \mathfrak{e}_{s}^{s+t-1} & \text { si } s+t \text { est impair } \\ \Sigma^{s+t} \mathfrak{D}(s) \mathfrak{e}_{s}^{s+t-1} & \text { si } s+t \text { est pair. }\end{cases}
$$

On convient d'alléger cette formule en écrivant $D_{s} \Sigma^{t} \mathbb{Z} / p=\Sigma^{s+t} D_{ \pm}(s) \mathfrak{e}_{s}^{s+t-1}$.

Démonstration. - D'après [24, 4.2.1], on a $\operatorname{St}_{s}\left(\Sigma^{k} \iota\right)=(-1)^{\frac{k(k-1) s}{2}} \Sigma^{k} \mathfrak{e}_{s}^{k}, \iota$ désignant le générateur de $\mathbb{Z} / p$. Le théorème 5.2 donne :

$$
\begin{aligned}
D_{s} \Sigma^{t} \mathbb{Z} / p & =D_{s} \Sigma^{1-s}\left(\Sigma^{s+t-1} \mathbb{Z} / p\right) \cong \Sigma R_{s}\left(\Sigma^{s+t-1} \mathbb{Z} / p\right) \\
& \cong \begin{cases}\Sigma D(s) \cdot \operatorname{St}_{s}\left(\Sigma^{s+t-1} \mathbb{Z} / p\right) & \text { si } s+t-1 \text { est pair, } \\
\Sigma \mathfrak{D}(s) \cdot \operatorname{St}_{s}\left(\Sigma^{s+t-1} \mathbb{Z} / p\right) & \text { si } s+t-1 \text { est impair },\end{cases} \\
& \cong \begin{cases}\Sigma^{s+t} D(s) \mathfrak{e}_{s}^{s+t-1} & \text { si } s+t \text { est impair, } \\
\Sigma^{s+t} \mathfrak{D}(s) \mathfrak{e}_{s}^{s+t-1} & \text { si } s+t \text { est pair. }\end{cases}
\end{aligned}
$$

Le corollaire est démontré.

5.2. Calcul de $\operatorname{Ext}_{\mathcal{M}}^{s}\left(\Sigma^{-t} \mathbb{Z} / p, L_{n, 3}\right)$. - Soit $N$ un module instable. La suite spectrale de Grothendieck associée à la composée des foncteurs

$$
\mathcal{M} \stackrel{D}{\longrightarrow} \stackrel{\operatorname{Hom}_{u}(-, N)}{\longrightarrow} V
$$

est de la forme :

$$
E_{2}^{u, v}=\operatorname{Ext}_{\mathcal{U}}^{u}\left(D_{v}(-), N\right) \Longrightarrow \operatorname{Ext}_{\mathcal{M}}^{u+v}(-, N) .
$$

On se sert du corollaire 5.3 et cette suite spectrale pour calculer les groupes d'extension $\operatorname{Ext}_{\mathcal{M}}^{s}\left(\Sigma^{-t} \mathbb{Z} / p, L_{n, 3}\right)$. On commence par le

LEMme 5.4. - Soit $M$ un module instable. On a

1. $\operatorname{Ext}_{u}^{s}\left(M, L_{n, 3}\right)=0$ si $s>n$;

2. $\operatorname{Ext}_{\mathscr{U}}^{s}\left(\Sigma^{d} M, L_{n, 3}\right)=0$ si $d>2 p^{s}-2$.

TOME $140-2012-\mathrm{N}^{\mathrm{O}} 2$ 
Démonstration. - La première assertion est conséquence directe de la résolution injective de $L_{n, 3}$. Pour la deuxième, on note $\tilde{\Sigma}$ le foncteur adjoint à droite $\mathrm{du}$ foncteur de suspension $\Sigma$. On a $\tilde{\Sigma} J(k)=J(k-1)$ pour $k \geq 1$ et $\tilde{\Sigma} J(0)=0$. De plus, si $L$ est un module instable réduit et $J$ est un module instable, on a $L \otimes \tilde{\Sigma} J \cong \tilde{\Sigma}(L \otimes J)$ [14]. Comme le module instable $L_{n-s}$ est réduit, on obtient

$$
\operatorname{Hom}_{U}\left(\Sigma^{d} M, L_{n-s} \otimes J\left(2 p^{s}-2\right)\right)=\operatorname{Hom}_{\mathcal{U}}\left(M, L_{n-s} \otimes \widetilde{\Sigma}^{d} J\left(2 p^{s}-2\right)\right)=0
$$

si $d>2 p^{s}-2$. Le lemme est démontré.

5.2.1. Les groupes $\operatorname{Ext}_{\mathcal{M}}^{n}\left(\Sigma^{2 p^{n}-2} \mathbb{Z} / p, L_{n, 3}\right)$ et $\operatorname{Ext}_{\mathcal{M}}^{n+1}\left(\Sigma^{2 p^{n-1}-2} \mathbb{Z} / p, L_{n, 3}\right)$. On calcule ensuite les groupes

$$
\operatorname{Ext}_{\mathcal{M}}^{n}\left(\Sigma^{2 p^{n}-2} \mathbb{Z} / p, L_{n, 3}\right) \text { et } \operatorname{Ext}_{\mathcal{M}}^{n+1}\left(\Sigma^{2 p^{n-1}-2} \mathbb{Z} / p, L_{n, 3}\right) .
$$

Proposition 5.5. - On a $\operatorname{Ext}_{\mathcal{M}}^{n}\left(\Sigma^{2 p^{n}-2} \mathbb{Z} / p, L_{n, 3}\right) \cong \mathbb{Z} / p$.

Démonstration. — On a la suite spectrale de Grothendieck :

$$
\operatorname{Ext}_{\mathcal{U}}^{u}\left(D_{v} \Sigma^{2 p^{n}-2} \mathbb{Z} / p, L_{n, 3}\right) \Longrightarrow \operatorname{Ext}_{\mathcal{M}}^{u+v}\left(\Sigma^{2 p^{n}-2} \mathbb{Z} / p, L_{n, 3}\right)
$$

D'après le corollaire 5.3 , on a

$$
D_{v} \Sigma^{2 p^{n}-2} \mathbb{Z} / p=\Sigma^{v+2 p^{n}-2} D_{ \pm}(v) \mathfrak{e}_{v}^{v+2 p^{n}-3} .
$$

On déduit du lemme 5.4 que

$$
\begin{aligned}
\operatorname{Ext}_{\mathcal{U}}^{u}\left(D_{v} \Sigma^{2 p^{n}-2} \mathbb{Z} / p, L_{n, 3}\right) & = \begin{cases}0 & \text { si } u \neq n, \\
\operatorname{Hom}_{\mathcal{U}}\left(D_{v} \Sigma^{2 p^{n}-2} \mathbb{Z} / p, J\left(2 p^{n}-2\right)\right) & \text { si } u=n .\end{cases} \\
& = \begin{cases}0 & \text { si }(u, v) \neq(n, 0), \\
\mathbb{Z} / p & \text { si }(u, v)=(n, 0) .\end{cases}
\end{aligned}
$$

Il suit que $\operatorname{Ext}_{\mathcal{M}}^{n}\left(\Sigma^{2 p^{n}-2} \mathbb{Z} / p, L_{n, 3}\right) \cong \operatorname{Ext}_{\mathcal{U}}^{n}\left(\Sigma^{2 p^{n}-2} \mathbb{Z} / p, L_{n, 3}\right) \cong \mathbb{Z} / p$.

Proposition 5.6. - On a $\operatorname{Ext}_{\mathcal{M}}^{n+1}\left(\Sigma^{2 p^{n-1}-2} \mathbb{Z} / p, L_{n, 3}\right) \cong \mathbb{Z} / p$.

Démonstration. - On a la suite spectrale de Grothendieck :

$$
\operatorname{Ext}_{\mathcal{U}}^{u}\left(D_{v} \Sigma^{2 p^{n-1}-2} \mathbb{Z} / p, L_{n, 3}\right) \Longrightarrow \operatorname{Ext}_{\mathcal{M}}^{u+v}\left(\Sigma^{2 p^{n-1}-2} \mathbb{Z} / p, L_{n, 3}\right) .
$$

D'après le corollaire 5.3 , on a

$$
D_{v} \Sigma^{2 p^{n-1}-2} \mathbb{Z} / p=\Sigma^{v+2 p^{n-1}-2} D_{ \pm}(v) \mathfrak{e}_{v}^{v+2 p^{n-1}-3} .
$$

- Si $v=0$ alors, pour tout $0 \leq i \leq n$, on a

$$
\operatorname{Hom}_{u}\left(\Sigma^{2 p^{n-1}-2} \mathbb{Z} / p, L_{n-i} \otimes J\left(2 p^{i}-2\right)\right)=0 .
$$


- Si $v=1$ alors $D_{1} \Sigma^{2 p^{n-1}-2} \mathbb{Z} / p=\Sigma^{2 p^{n-1}-1} D(1) \mathfrak{e}_{1}^{2 p^{n-1}-2}$, d'où

$$
\begin{aligned}
\operatorname{Ext}_{\mathscr{U}}^{u}\left(D_{1} \Sigma^{2 p^{n-1}-2} \mathbb{Z} / p, L_{n, 3}\right) & = \begin{cases}0 & \text { si } u \neq n, \\
\operatorname{Hom}_{\mathcal{U}}\left(D_{1} \Sigma^{2 p^{n-1}-2} \mathbb{Z} / p, J\left(2 p^{n}-2\right)\right) & \text { si } u=n\end{cases} \\
& = \begin{cases}0 & \text { si } u \neq n, \\
\mathbb{Z} / p & \text { si } u=n .\end{cases}
\end{aligned}
$$

Ici $\operatorname{Hom}_{\mathcal{U}}\left(D_{1} \Sigma^{2 p^{n-1}-2} \mathbb{Z} / p, J\left(2 p^{n}-2\right)\right)=\mathbb{Z} / p$ car le sous-espace des éléments de degré $2 p^{n}-2$ de $\Sigma^{2 p^{n-1}-1} D(1) \mathfrak{e}_{1}^{2 p^{n-1}-2}$ est engendré par $\Sigma^{2 p^{n-1}-1} X Y^{p^{n-1}}$.

- Si $v \geq 2$ alors la connectivité de

$$
D_{v} \Sigma^{2 p^{n-1}-2} \mathbb{Z} / p=\Sigma^{2 p^{n-1}-2+v} D_{ \pm}(v) \mathfrak{e}_{v}^{2 p^{n-1}-3+v}
$$

est supérieure ou égale au degré de $\Sigma^{2 p^{n-1}-2+v} \mathfrak{e}_{v}^{2 p^{n-1}-3+v}$, soit $\left(2 p^{n-1}-2+v\right)+\left(2 p^{n-1}-3+v\right)\left(p^{v}-1\right)=\left(2 p^{n-1}-3+v\right) p^{v}+1>2 p^{n}-2$.

On en déduit que

$$
\operatorname{Ext}_{u}^{u}\left(D_{v} \Sigma^{2 p^{n-1}-2} \mathbb{Z} / p, L_{n, 3}\right)= \begin{cases}0 & \text { si }(u, v) \neq(n, 1), \\ \mathbb{Z} / p & \text { si }(u, v)=(n, 1) .\end{cases}
$$

D'où $\operatorname{Ext}_{\mathcal{M}}^{n+1}\left(\Sigma^{2 p^{n-1}-2} \mathbb{Z} / p, L_{n, 3}\right) \cong \operatorname{Ext}_{\mathcal{U}}^{n}\left(D_{1} \Sigma^{2 p^{n-1}-2} \mathbb{Z} / p, L_{n, 3}\right) \cong \mathbb{Z} / p$.

5.2.2. Les groupes $\operatorname{Ext}_{\mathcal{M}}^{s}\left(\Sigma^{-t} \mathbb{Z} / p, L_{n, 3}\right)$ pour $s-t \geq 2 p^{n-2}+n$. - On va montrer la nullité des groupes d'extension $\operatorname{Ext}_{\mathcal{M}}^{s}\left(\Sigma^{-t} \mathbb{Z} / p, L_{n, 3}\right)$ pour $s-t \geq$ $2 p^{n-2}+n$ et $(s, t) \notin\left\{\left(n, 2-2 p^{n}\right),\left(n+1,2-2 p^{n-1}\right)\right\}$.

Pour $t \geq 0$, considérons le morphisme

$$
f: \operatorname{Hom}_{u}\left(D_{1} \Sigma^{-t} \mathbb{Z} / p, L_{1} \otimes J\left(2 p^{n-1}-2\right)\right) \rightarrow \operatorname{Hom}_{\mathcal{U}}\left(D_{1} \Sigma^{-t} \mathbb{Z} / p, J\left(2 p^{n}-2\right)\right),
$$

induit par le morphisme $f_{n-1, n}: L_{1} \otimes J\left(2 p^{n-1}-2\right) \rightarrow J\left(2 p^{n}-2\right)$. D'après le corollaire 5.3 , on a

$$
D_{1} \Sigma^{-t} \mathbb{Z} / p= \begin{cases}\Sigma^{1-t} D(1) \mathfrak{e}_{1}^{-t} & \text { si } t \text { est pair } \\ \Sigma^{1-t} \mathfrak{D}(1) \mathfrak{e}_{1}^{-t} & \text { si } t \text { est impair. }\end{cases}
$$

On obtient donc une inclusion $D_{1} \Sigma^{-t} \mathbb{Z} / p \hookrightarrow \Sigma^{1-t} D(1)$ dont le quotient est fini. Il s'ensuit que cette inclusion induit un isomorphisme

$$
\left(D_{1} \Sigma^{-t} \mathbb{Z} / p: L_{1}\right)_{u} \cong\left(\Sigma^{1-t} D(1): L_{1}\right) u .
$$

Comme $\left(D(1): L_{1}\right) u \cong D(1)$, on obtient

$$
\operatorname{Hom}_{u}\left(D_{1} \Sigma^{-t} \mathbb{Z} / p, L_{1} \otimes J\left(2 p^{n-1}-2\right)\right) \cong \operatorname{Hom}_{u}\left(\Sigma^{1-t} D(1), J\left(2 p^{n-1}-2\right)\right) .
$$

On vérifie que le domaine et la source de $f$ sont soit $\{0\}$ soit $\mathbb{Z} / p$. 
Lemme 5.7. - Supposons que $\operatorname{Hom}_{\mathcal{u}}\left(D_{1} \Sigma^{-t} \mathbb{Z} / p, L_{1} \otimes J\left(2 p^{n-1}-2\right)\right) \cong \mathbb{Z} / p$. Alors $f$ est un isomorphisme.

Démonstration. - On peut choisir $i \geq 0$ et $\epsilon=0,1$ tels que le degré de $\Sigma^{1-t} X^{\epsilon} Y^{i}$ soit $2 p^{n-1}-2$, i.e.

$$
(t-1)+\left(2 p^{n-1}-2\right)=2 i(p-1)-\epsilon \geq 0 .
$$

Il s'ensuit que $\Sigma^{1-t} X^{\epsilon} Y^{i+p^{n-1}}$ est un élément de degré $2 p^{n}-2$ de $D_{1} \Sigma^{-t} \mathbb{Z} / p$, donc la source de $f$ est aussi non nulle. En effet,

- Si $t=2 k \leq 0$ alors $\epsilon=1$ et $k+\left(p^{n-1}-1\right)=i(p-1)>0$. Ceci implique l'élément $\Sigma^{1-2 k} X Y^{i+p^{n-1}} \in \Sigma^{1-2 k} M_{1} \cdot Y^{-k}$ est de degré $2 p^{n}-2$. .

- Si $t=2 k-1 \leq 0$ alors $\epsilon=0$ et $(k-1)+\left(p^{n-1}-1\right)=i(p-1) \geq 0$. Ceci implique l'élément $\Sigma^{2-2 k} Y^{i+p^{n-1}} \in \Sigma^{2-2 k} L_{1} \cdot Y^{-k}$ est de degré $2 p^{n}-2$.

En particulier, on a $0 \leq i(p-1)-\epsilon \leq p^{n-1}-2$.

Soit $\alpha: \Sigma^{1-t} D(1) \rightarrow J\left(2 p^{n-1}-2\right)$ le générateur représenté par $\Sigma^{1-t} X^{\epsilon} Y^{i}$. On considère le composé suivant, noté $\beta$, qui est un élément de $\operatorname{Hom}_{\mathcal{U}}\left(D_{1} \Sigma^{-t} \mathbb{Z} / p, L_{1} \otimes J\left(2 p^{n-1}-2\right)\right)$ :

$D_{1} \Sigma^{-t} \mathbb{Z} / p \hookrightarrow D(1) \otimes \Sigma^{1-t} \mathbb{Z} / p \stackrel{\delta \otimes \mathrm{id}}{\longrightarrow} D(1) \otimes D(1) \otimes \Sigma^{1-t} \mathbb{Z} / p \stackrel{\pi \otimes \alpha}{\longrightarrow} L_{1} \otimes J\left(2 p^{n-1}-2\right)$ où $\delta: D(1) \rightarrow D(1) \otimes D(1)$ est donné par $\delta\left(x_{1}^{\epsilon} y_{1}^{(p-1) i}\right)=\left(x_{1}+x_{2}\right)^{\epsilon}\left(y_{1}+y_{2}\right)^{(p-1) i}$ et $\pi: D(1) \rightarrow L_{1}$ est la projection canonique. Or, le coefficient de $Y_{1}^{p^{n-1}} X_{2}^{\epsilon} Y_{2}^{i}$ dans le développement de

$$
\delta\left(X_{1}^{\epsilon} Y_{1}^{p^{n-1}+i}\right)=\left(x_{1}+x_{2}\right)^{\epsilon}\left(y_{1}+y_{2}\right)^{(p-1)\left(p^{n-1}+i\right)-\epsilon}
$$

est $\left(\begin{array}{c}(p-1)\left(p^{n-1}+i\right)-\epsilon \\ (p-1) p^{n-1}\end{array}\right)$ qui est non nul puisque

$$
(p-1) p^{n-1} \leq(p-1) p^{n-1}+((p-1) i-\epsilon) \leq(p-1) p^{n-1}+\left(p^{n-1}-2\right) .
$$

Il s'ensuit que le composé

$$
D_{1} \Sigma^{-t} \mathbb{Z} / p \stackrel{\beta}{\rightarrow} L_{1} \otimes J\left(2 p^{n-1}-2\right) \rightarrow J\left(2 p^{n}-2\right)
$$

est non nul. Le morphisme $f$ est ainsi un isomorphisme du groupe $\mathbb{Z} / p$.

LEMme 5.8. - Supposons $t \neq 2-2 p^{n-1}$ et $\operatorname{Hom}_{u}\left(D_{1} \Sigma^{-t} \mathbb{Z} / p, J\left(2 p^{n}-2\right)\right) \cong$ $\mathbb{Z} / p$. Alors $f$ est un isomorphisme.

Démonstration. - Il suffit de montrer que le groupe $\operatorname{Hom}_{\mathcal{U}}\left(D_{1} \Sigma^{-t} \mathbb{Z} / p, L_{1} \otimes\right.$ $\left.J\left(2 p^{n-1}-2\right)\right)$ est non nul en considérant les deux cas suivants :

- Si $t=2 k$ alors ils existent $i \geq 0$ et $\epsilon=0,1$ tels que le degré de $\Sigma^{1-2 k} X^{\epsilon} Y^{i-k} \in \Sigma^{1-2 k} D(1) \cdot Y^{-k}$ soit $2 p^{n}-2$, i.e

$$
(2 k-1)+2 k(p-1)+\left(2 p^{n}-2\right)=2 i(p-1)-\epsilon \geq 0 .
$$


Il suit $\epsilon=1$ et $k p+\left(p^{n}-1\right)=i(p-1)>0$. Il suit encore $k=(p-1) \kappa \leq 0$ et $\kappa p+\frac{p^{n}-1}{p-1}=i>0$. Il s'ensuit $i=\iota p+1$ et $k+\left(p^{n-1}-1\right)=\iota(p-1) \geq 0$. Comme $k \neq 1-p^{n-1}$, on a $\iota>0$. On en déduit que $\Sigma^{1-2 k} X Y^{\iota}$ est un élément de degré $2 p^{n-1}-2$ de $\Sigma^{1-2 k} D(1)$. Ceci implique le groupe $\operatorname{Hom}_{u}\left(D_{1} \Sigma^{-2 k} \mathbb{Z} / p, L_{1} \otimes\right.$ $\left.J\left(2 p^{n-1}-2\right)\right)$ est non nul.

- $\quad$ Si $t=2 k-1, k \leq 0$, alors ils existent $i \geq 0$ et $\epsilon=0,1$ tels que le degré de $\Sigma^{2-2 k} X^{\epsilon} Y^{i-k} \in \Sigma^{2-2 k} L_{1} \cdot Y^{-k}$ soit $2 p^{n}-2$, i.e

$$
(2 k-2)+2 k(p-1)+\left(2 p^{n}-2\right)=2 i(p-1)-\epsilon>0 .
$$

Il suit $\epsilon=0$ et $k p-1+\left(p^{n}-1\right)=i(p-1)>0$. Il suit encore $k=(p-1) \kappa+1 \leq 0$ et $\kappa p+1+\frac{p^{n}-1}{p-1}=i>0$. Il s'ensuit $i=\iota p+2$ et $k-1+\left(p^{n-1}-1\right)=\iota(p-1) \geq 0$. On en déduit que $\Sigma^{2-2 k} Y^{\iota}$ est un élément de degré $2 p^{n-1}-2$ de $\Sigma^{2-2 k} D(1)$. Ceci implique le groupe $\operatorname{Hom}_{\mathcal{U}}\left(D_{1} \Sigma^{-2 k+1} \mathbb{Z} / p, L_{1} \otimes J\left(2 p^{n-1}-2\right)\right)$ est non nul.

Proposition 5.9. - Supposons $n \geq 2,(s, t) \neq\left(n, 2-2 p^{n}\right)$ et $(s, t) \neq(n+$ $\left.1,2-2 p^{n-1}\right)$. Alors le groupe $\operatorname{Ext}_{\mathcal{M}}^{s}\left(\Sigma^{-t} \mathbb{Z} / p, L_{n, 3}\right)$ est nul dès que $s-t \geq$ $2 p^{n-2}+n$.

Démonstration. - On a la suite spectrale de Grothendieck :

$$
\operatorname{Ext}_{\mathcal{U}}^{u}\left(D_{s-u} \Sigma^{-t} \mathbb{Z} / p, L_{n, 3}\right) \Longrightarrow \operatorname{Ext}_{\mathcal{M}}^{s}\left(\Sigma^{-t} \mathbb{Z} / p, L_{n, 3}\right) \text {. }
$$

On va montrer que $\operatorname{Ext}_{u}^{u}\left(D_{s-u} \Sigma^{-t} \mathbb{Z} / p, L_{n, 3}\right)=0$ pour $0 \leq u \leq n$. D'après le corollaire 5.3 , on a

$$
D_{s-u} \Sigma^{-t} \mathbb{Z} / p=\Sigma^{s-t-u} D_{ \pm}(s-u) \mathfrak{e}_{s-u}^{s-t-u-1} .
$$

Considérons les trois cas suivants :

1. Pour $0 \leq u \leq n-2$, on a $s-t-u>2 p^{n-2}-2$. On en déduit que le groupe $\operatorname{Ext}_{\mathcal{U}}^{u}\left(D_{s-u} \Sigma^{-t} \mathbb{Z} / p, L_{n, 3}\right)$ est nul pour tout $0 \leq u \leq n-2$.

2. Pour $u=n-1$, le groupe $\operatorname{Ext}_{u}^{n-1}\left(D_{s-n+1} \Sigma^{-t} \mathbb{Z} / p, L_{n, 3}\right)$ est le noyau du morphisme

$f: \operatorname{Hom}_{\mathcal{U}}\left(D_{s-n+1} \Sigma^{-t} \mathbb{Z} / p, L_{1} \otimes J\left(2 p^{n-1}-2\right)\right) \rightarrow \operatorname{Hom}_{\mathcal{U}}\left(D_{s-n+1} \Sigma^{-t} \mathbb{Z} / p, J\left(2 p^{n}-2\right)\right)$ qui est induit par le morphisme $f_{n-1, n}: L_{1} \otimes J\left(2 p^{n-1}-2\right) \rightarrow J\left(2 p^{n}-2\right)$.

(a) Si $s=n-1$, le morphisme $f$ devient

$$
\operatorname{Hom}_{\mathcal{U}}\left(\Sigma^{-t} \mathbb{Z} / p, L_{1} \otimes J\left(2 p^{n-1}-2\right)\right) \rightarrow \operatorname{Hom}_{\mathcal{U}}\left(\Sigma^{-t} \mathbb{Z} / p, J\left(2 p^{n}-2\right)\right)
$$

avec $t \leq-2 p^{n-2}-1$. Le domaine de ce morphisme est trivial.

(b) Si $s=n$, le morphisme $f$ devient

$$
\operatorname{Hom}_{\mathcal{U}}\left(D_{1} \Sigma^{-t} \mathbb{Z} / p, L_{1} \otimes J\left(2 p^{n-1}-2\right)\right) \rightarrow \operatorname{Hom}_{u}\left(D_{1} \Sigma^{-t} \mathbb{Z} / p, J\left(2 p^{n}-2\right)\right)
$$

avec $t \leq-2 p^{n-2}$. Si le domaine de $f$ est non nul, $f$ est un isomorphisme d'après le lemme 5.7. 
(c) Si $s \geq n+1$, d'après le corollaire 6.23, la connectivité de la dévision $\left(D_{s-n+1} \Sigma^{-t} \mathbb{Z} / p: L_{1}\right)$ est $\geq p^{s-n}(s-t-n)+1$. Comme $s-t \geq$ $2 p^{n-2}+n$, celle-ci est supérieure à $2 p^{n-1}-2$. Le domaine de $f$ est nul.

3. Pour $u=n$, comme le cas précédent, le groupe $\operatorname{Ext}_{\mathcal{U}}^{n}\left(D_{s-n} \Sigma^{-t} \mathbb{Z} / p, L_{n, 3}\right)$ est le conoyau du morphisme

$f^{\prime}: \operatorname{Hom}_{\mathcal{U}}\left(D_{s-n} \Sigma^{-t} \mathbb{Z} / p, L_{1} \otimes J\left(2 p^{n-1}-2\right)\right) \rightarrow \operatorname{Hom}_{\mathcal{U}}\left(D_{s-n} \Sigma^{-t} \mathbb{Z} / p, J\left(2 p^{n}-2\right)\right)$.

(a) Si $s=n$, le morphisme $f^{\prime}$ devient

$$
\operatorname{Hom}_{\mathcal{U}}\left(\Sigma^{-t} \mathbb{Z} / p, L_{1} \otimes J\left(2 p^{n-1}-2\right) \rightarrow \operatorname{Hom}_{u}\left(\Sigma^{-t} \mathbb{Z} / p, J\left(2 p^{n}-2\right)\right) .\right.
$$

Le domaine de ce morphisme est trivial car $t \neq 2-2 p^{n}$.

(b) Si $s=n+1$, alors $t \neq 2-2 p^{n-1}$ et le morphisme $f^{\prime}$ devient

$$
\operatorname{Hom}_{u}\left(D_{1} \Sigma^{-t} \mathbb{Z} / p, L_{1} \otimes J\left(2 p^{n-1}-2\right)\right) \rightarrow \operatorname{Hom}_{\mathcal{U}}\left(D_{1} \Sigma^{-t} \mathbb{Z} / p, J\left(2 p^{n}-2\right)\right)
$$

avec $t \leq 1-2 p^{n-2}$. Si la source de $f^{\prime}$ est non nulle, alors $f^{\prime}$ est un isomorphisme du groupe $\mathbb{Z} / p$ d'après le lemme 5.8.

(c) Si $s=n+2$, le morphisme $f^{\prime}$ devient

$$
\operatorname{Hom}_{\mathcal{U}}\left(D_{2} \Sigma^{-t} \mathbb{Z} / p, L_{1} \otimes J\left(2 p^{n-1}-2\right)\right) \rightarrow \operatorname{Hom}_{\mathcal{U}}\left(D_{2} \Sigma^{-t} \mathbb{Z} / p, J\left(2 p^{n}-2\right)\right)
$$

avec $t \leq 2-2 p^{n-2}$. On a $D_{2} \Sigma^{-t} \mathbb{Z} / p \subset \Sigma^{2-t}(D(2) \oplus \mathfrak{D}(2)) \mathfrak{e}_{2}^{1-t}$. Supposons qu'il existe $a \in D(2) \oplus \mathfrak{D}(2)$ tel que le degré de $\Sigma^{2-t} a$. $\mathfrak{e}_{2}^{1-t}$ soit $2 p^{n}-2$, i.e. $\operatorname{deg} a+(2-t)+(1-t)\left(p^{2}-1\right)=2 p^{n}-2$, soit $\operatorname{deg} a+(1-t) p^{2}=2 p^{n}-3$. Comme $t \leq 2-2 p^{n-2}$, il suit $\operatorname{deg} a \leq p^{2}-3$. D'autre part, d'après le corollaire 6.12,

$$
D(2) \oplus \mathfrak{D}(2) \cong \Lambda\left(\widetilde{\mathbf{M}}_{2,0}, \widetilde{\mathbf{M}}_{2,1}\right) \otimes \mathbb{F}_{p}\left[\mathfrak{e}_{2}, \mathbf{Q}_{2,1}\right]
$$

où $\operatorname{deg} \mathfrak{e}_{2}=p^{2}-1, \operatorname{deg} \mathbf{Q}_{2,1}=2\left(p^{2}-p\right), \operatorname{deg} \widetilde{\mathbf{M}}_{2,0}=p^{2}-2$ et $\operatorname{deg} \widetilde{\mathbf{M}}_{2,1}=p^{2}-2 p$. On en déduit que soit $\operatorname{deg} a=0$, soit $\operatorname{deg} a=$ $p^{2}-2 p$.

- $\quad$ Si $\operatorname{deg} a=0$, on a $(1-t) p^{2}=2 p^{n}-3$ ce qui est absurde puisque 3 n'est pas divisible par $p^{2}$.

- $\quad$ Si $\operatorname{deg} a=p^{2}-2 p$, on a $\left(p^{2}-2 p\right)+(1-t) p^{2}=2 p^{n}-3$. Ceci implique $p=3$, d'où $3(1-t)=2.3^{n-1}-2$ ce qui est encore impossible.

On a ainsi vérifié que le groupe $\operatorname{Hom}_{\mathcal{U}}\left(D_{2} \Sigma^{-t} \mathbb{Z} / p, J\left(2 p^{n}-2\right)\right)$ est trivial. 
(d) Si $s \geq n+3$, la connectivité de $D_{s-n} \Sigma^{-t} \mathbb{Z} / p$ est supérieure ou égale au degré de $\Sigma^{s-t-n} \mathfrak{e}_{s-n}^{s-t-n-1}$. Pour $n \geq 2$ et $s-t \geq 2 p^{n-2}+n$, on a

$$
\begin{aligned}
\operatorname{deg} \Sigma^{s-t-n} \mathfrak{e}_{s-n}^{s-t-n-1} & =p^{s-n}(s-t-n-1)+1 \\
& \geq p^{3}\left(2 p^{n-2}-1\right)+1 \\
& =\left(2 p^{n}(p-1)-p^{3}+3\right)+\left(2 p^{n}-2\right) \\
& >2 p^{n}-2 .
\end{aligned}
$$

Le domaine de $f^{\prime}$ est donc trivial.

En résumé, on a montré que $\operatorname{Ext}_{\mathcal{u}}^{u}\left(D_{s-u} \Sigma^{-t} \mathbb{Z} / p, L_{n, 3}\right)=0$ pour tout $0 \leq u \leq n$. La proposition suit.

La proposition 5.9 est en fait aussi valable pour $n=1$.

Proposition 5.10. - Supposons $(s, t) \neq(1,2-2 p)$ et $(s, t) \neq(2,0)$. Alors le groupe $\operatorname{Ext}_{M}^{s}\left(\Sigma^{-t} \mathbb{Z} / p, L_{1,3}\right)$ est nul dès que $s-t \geq 2$.

Démonstration. — On a la suite spectrale de Grothendieck :

$$
\operatorname{Ext}_{\mathcal{U}}^{u}\left(D_{s-u} \Sigma^{-t} \mathbb{Z} / p, L_{1,3}\right) \Longrightarrow \operatorname{Ext}_{\mathcal{M}}^{s}\left(\Sigma^{-t} \mathbb{Z} / p, L_{1,3}\right)
$$

On va montrer que $\operatorname{Ext}_{\mathcal{U}}^{u}\left(D_{s-u} \Sigma^{-t} \mathbb{Z} / p, L_{1,3}\right)=0$ pour $0 \leq u \leq 1$ en utilisant la résolution injective $L_{1,3} \hookrightarrow L_{1} \rightarrow J(2 p-2)$.

Pour $u=0$, on a $\operatorname{Ext}_{\mathcal{u}}^{u}\left(D_{s-u} \Sigma^{-t} \mathbb{Z} / p, L_{1,3}\right)=\operatorname{Hom}_{\mathcal{U}}\left(D_{s} \Sigma^{-t} \mathbb{Z} / p, L_{1,3}\right)$ qui est nul puisque $D_{s} \Sigma^{-t} \mathbb{Z} / p$ est une suspension d'après le corollaire 5.3. Pour $u=1$, comme $D_{s-1} \Sigma^{-t} \mathbb{Z} / p$ est encore une suspension, le groupe $\operatorname{Hom}_{u}\left(D_{s-1} \Sigma^{-t} \mathbb{Z} / p, L_{1,3}\right)$ est nul. D'où

$$
\operatorname{Ext}_{\mathcal{U}}^{1}\left(D_{s-1} \Sigma^{-t} \mathbb{Z} / p, L_{1,3}\right) \cong \operatorname{Hom}_{\mathcal{U}}\left(D_{s-1} \Sigma^{-t} \mathbb{Z} / p, J(2 p-2)\right) .
$$

On vérifie sans peine que, pour $s-t \geq 2$, le groupe $\operatorname{Hom}_{u}\left(D_{s-1} \Sigma^{-t} \mathbb{Z} / p, J(2 p-\right.$ $2)$ ) est nul si $(s, t) \neq(1,2-2 p)$ ou $(s, t) \neq(2,0)$. La proposition suit.

Remarque 5.11. - Afin de pousser plus loin le calcul de $\operatorname{Ext}_{\mathcal{M}}^{*}\left(\Sigma^{*} \mathbb{Z} / p, L_{n, 3}\right)$ dans cette direction, on a besoin d'étudier l'espace vectoriel gradué $\left(D(s): L_{k}\right) u$ pour $k \geq 2$, ceci sera fait ailleurs. 


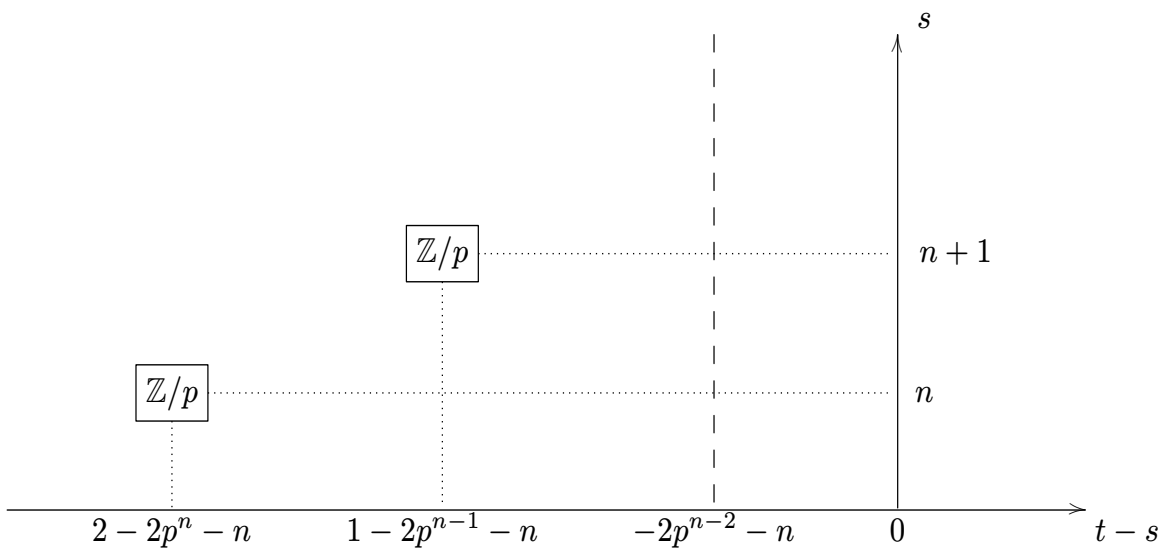

Figure 1. $\operatorname{Ext}_{\mathcal{M}}^{s}\left(\Sigma^{-t} \mathbb{Z} / p, L_{n, 3}\right), s-t \geq 2 p^{n-2}+n$

5.3. Cohomotopie de $L(n, 3)$. - Voici un résultat partiel sur les groupes d'homotopie du spectre $L(n, 3)$.

ThÉORÈme 5.12. - Supposons $k \geq 2 p^{n-2}+n$. Alors

$$
\left[L(n, 3), S^{k}\right]= \begin{cases}\mathbb{Z} / p & \text { si } k=2 p^{n}-2+n \text { où } k=2 p^{n-1}-1+n \\ 0 & \text { sinon. }\end{cases}
$$

Démonstration. - La suite spectrale d'Adams pour la cohomotopie $\left[L(n, 3), S^{k}\right]$, $k \in \mathbb{Z}$ est de la forme :

$$
E_{2}^{s, t}=\operatorname{Ext}_{\mathcal{M}}^{s}\left(\Sigma^{-t} \mathbb{Z} / p, L_{n, 3}\right) \Longrightarrow\left[L(n, 3), S^{s-t}\right], \quad d_{r}: E_{r}^{s, t} \rightarrow E_{r}^{s+r, t+r-1} .
$$

Pour $s-t \geq 2 p^{n-2}+n$, on déduit des propositions 5.5, 5.6, 5.9 et 5.10 que

$$
E_{\infty}^{s, t}= \begin{cases}\mathbb{Z} / p & \text { si }(s, t)=\left(n, 2-2 p^{n}\right) \text { ou }(s, t)=\left(n+1,2-2 p^{n-1}\right), \\ 0 & \text { sinon. }\end{cases}
$$

Le théorème suit.

\section{6. $\left(D(n): L_{1}\right)_{u}$ en tant qu'espace vectoriel gradué}

L'objectif de cette section est de déterminer $\left(D(n): L_{1}\right) u$ en tant qu'espace vectoriel gradué. En particulier, pour $s+t \geq 1$, la connectivité de $\left(D_{s} \Sigma^{t} \mathbb{Z} / p: L_{1}\right)$ u sera déterminée. Cette connectivité a été utilisée dans la démonstration de la proposition $5.9(\operatorname{cas} 2 \mathrm{c})$. 
6.1. Invariants modulaires. - Afin de décrire la structure de $D(n)$, nous allons rappeler certains invariants modulaires de $\mathrm{GL}_{n}$ définis par Dickson [6] et Mui [18].

Rappelons que $\mathrm{GL}_{n}$ agit à gauche sur $H^{*} V_{n} \cong \Lambda\left(x_{1}, \ldots, x_{n}\right) \otimes \mathbb{F}_{p}\left[y_{1}, \ldots, y_{n}\right]$ comme suit. Etant donnés $g=\left(g_{i, j}\right) \in \mathrm{GL}_{n}$ et $f \in H^{*} V_{n}$, on a

$$
g \cdot f\left(x_{1}, y_{1}, \cdots, x_{n}, y_{n}\right)=f\left(g \cdot x_{1}, g \cdot x_{n}, \cdots, g \cdot x_{n}, g \cdot g \cdot y_{n}\right)
$$

avec $g \cdot x_{j}=\sum_{i=1}^{n} g_{i, j} x_{i}$ et $g \cdot y_{j}=\sum_{i=1}^{n} g_{i, j} y_{i}$ pour $1 \leq j \leq n$.

Appelons $f$ invariant de $\mathrm{GL}_{n}$ par rapport à $c: \mathrm{GL}_{n} \rightarrow \mathbb{F}_{p}^{*}$ si $g \cdot f=c(g) f$ pour tout $g \in \mathrm{GL}_{n}$. A la suite de Dickson [6], on pose

$$
\left[e_{1}, \ldots, e_{n}\right]=\left|\begin{array}{ccc}
y_{1}^{p^{e_{1}}} & \cdots & y_{n}^{p^{e_{1}}} \\
\vdots & \ddots & \vdots \\
y_{1}^{p^{e_{n}}} & \cdots & y_{n}^{p_{n}}
\end{array}\right|
$$

pour toute suite d'entiers naturels $\left(e_{1}, \ldots, e_{n}\right)$. Les $\left[e_{1}, \ldots, e_{n}\right]$ sont invariants par rapport à la représentation déterminant de $\mathrm{GL}_{n}$, i.e.

$$
g \cdot\left[e_{1}, \ldots, e_{n}\right]=\operatorname{det}(g)\left[e_{1}, \ldots, e_{n}\right]
$$

pour tout $g \in \mathrm{GL}_{n}$. En particulier, pour $0 \leq s \leq n$, on pose

$$
\mathbf{L}_{n, s}=[0, \ldots, \hat{s}, \ldots, n], \quad \mathbf{L}_{n}=\mathbf{L}_{n, n}=[0, \ldots, n-1]
$$

Lemme 6.1 ([6, 18]). - 1. Soit $f \in \mathbb{F}_{p}\left[y_{1}, \ldots, y_{n}\right]$ un $\mathrm{GL}_{n}$-invariant par rapport à det ${ }^{d}, 1 \leq d \leq p-2$. Alors $\mathbf{L}_{n}^{d}$ divise $f$.

2. Soit $f \in \Lambda^{k}\left(x_{1}, \ldots, x_{n}\right) \otimes \mathbb{F}_{p}\left[y_{1}, \ldots, y_{n}\right], 1 \leq k \leq n$, un $\mathrm{GL}_{n}$-invariant par rapport à $\operatorname{det}^{d}, 1 \leq d \leq p-1$. Alors $\mathbf{L}_{n}^{d-1}$ divise $f$.

D'après ce lemme, $\mathbf{L}_{n, s}$ est divisible par $\mathbf{L}_{n}$. On a l'invariant de Dickson $\mathbf{Q}_{n, s}=\frac{\mathbf{L}_{n, s}}{\mathbf{L}_{n}}$. Le degré de $\mathbf{Q}_{n, s}$ est $2\left(p^{n}-p^{s}\right)$. Notons que $\mathbf{Q}_{n, 0}=\mathbf{L}_{n}^{p-1}$ et $\mathbf{Q}_{n, n}=1$.

A la suite de [18], on pose

$$
\mathbf{V}_{k}=\mathbf{V}_{k}\left(y_{1}, \ldots, y_{k}\right)=\prod_{\lambda_{i} \in \mathbb{F}_{p}}\left(\lambda_{1} y_{1}+\cdots+\lambda_{k-1} y_{k-1}+y_{k}\right) .
$$

On a les relations suivantes entre les $\mathbf{L}_{n}, \mathbf{Q}_{n, s}$ et $\mathbf{V}_{k}$ :

$$
\begin{aligned}
\mathbf{L}_{n} & =\mathbf{V}_{1} \cdots \mathbf{V}_{n}, \\
\mathbf{Q}_{n, s} & =\mathbf{Q}_{n-1, s} \mathbf{V}_{n}^{p-1}+\mathbf{Q}_{n-1, s-1}, \\
\mathbf{V}_{k+1} & =\sum_{s=0}^{k}(-1)^{s+k} \mathbf{Q}_{k, s} y_{k+1}^{p^{s}} .
\end{aligned}
$$

TOME $140-2012-\mathrm{N}^{\mathrm{O}} 2$ 
Pour $0 \leq s \leq n-1$, posons

$$
\mathbf{M}_{n, s}=\left|\begin{array}{ccc}
x_{1} & \cdots & x_{n} \\
y_{1} & \cdots & y_{n} \\
\vdots & \ddots & \vdots \\
y_{1}^{p^{s-1}} & \cdots & y_{n}^{p^{s-1}} \\
y_{1}^{p^{s+1}} & \cdots & y_{n}^{p^{s+1}} \\
\vdots & \ddots & \vdots \\
y_{1}^{p^{n-1}} & \cdots & y_{n}^{p^{n-1}}
\end{array}\right|
$$

On a évidemment que $\mathbf{M}_{n, s}$ est invariant par rapport à la représentation déterminant de $\mathrm{GL}_{n}$. De plus, d'après [18, I.4.12], si $s \leq n-2$, on a

$$
\mathbf{M}_{n, s}=\mathbf{M}_{n-1, s} \mathbf{V}_{n}+\mathbf{M}_{n, n-1} \mathbf{Q}_{n-1, s} \text {. }
$$

Soient $0 \leq s_{1}<\cdots<s_{j} \leq n-1$. D'après le lemme $6.1, \mathbf{L}_{n}^{j-1}$ divise le produit $\mathbf{M}_{n, s_{1}} \cdots \mathbf{M}_{n, s_{j}}$. On a alors l'invariant de Mui :

$$
\mathbf{M}_{n ; s_{1}, \ldots, s_{j}}=(-1)^{\left(\begin{array}{c}
j \\
2
\end{array}\right)} \frac{\mathbf{M}_{n, s_{1}} \cdots \mathbf{M}_{n, s_{j}}}{\mathbf{L}_{n}^{j-1}} .
$$

Le degré de $\mathbf{M}_{n ; s_{1}, \ldots, s_{j}}$ est $\frac{2\left(p^{n}-1\right)}{p-1}+\left(1-2 p^{s_{1}}\right)+\cdots+\left(1-2 p^{s_{j}}\right)$. Si l'ensemble $\left\{s_{1}, \ldots, s_{j}\right\}$ est vide, on convient de poser $\mathbf{M}_{n, \varnothing}=\mathbf{L}_{n}$.

On note $\mathrm{SL}_{n}$ le sous-groupe de $\mathrm{GL}_{n}$ des matrices de déterminant 1.

ThÉORÈmE 6.2 ([6]). - On a

1. $\mathbb{F}_{p}\left[y_{1}, \ldots, y_{n}\right]^{\mathrm{SL}_{n}}=\mathbb{F}_{p}\left[\mathbf{L}_{n}, \mathbf{Q}_{n, 1}, \ldots, \mathbf{Q}_{n, n-1}\right] ;$

2. $\mathbb{F}_{p}\left[y_{1}, \ldots, y_{n}\right]^{\mathrm{GL}_{n}}=\mathbb{F}_{p}\left[\mathbf{Q}_{n, 0}, \ldots, \mathbf{Q}_{n, n-1}\right]$.

THÉORÈme 6.3 ([18]). — On a

1. $\left(\Lambda\left(x_{1}, \ldots, x_{n}\right) \otimes \mathbb{F}_{p}\left[y_{1}, \ldots, y_{n}\right]\right)^{\mathrm{SL}_{n}}$ est un $\mathbb{F}_{p}\left[y_{1}, \ldots, y_{n}\right]^{\mathrm{SL}_{n}}$-module libre de base

$$
\left\{1, \quad \mathbf{M}_{n ; s_{1}, \ldots, s_{j}}\right\}
$$

avec $0 \leq s_{1}<\cdots<s_{j} \leq n-1 ;$

2. $\left(\Lambda\left(x_{1}, \ldots, x_{n}\right) \otimes \mathbb{F}_{p}\left[y_{1}, \ldots, y_{n}\right]\right)^{\mathrm{GL}_{n}}$ est un $\mathbb{F}_{p}\left[y_{1}, \ldots, y_{n}\right]^{\mathrm{GL}_{n}}$-module libre de base

$$
\left\{1, \quad \mathbf{M}_{n ; s_{1}, \ldots, s_{j}} \mathbf{L}_{n}^{p-2}\right\}
$$

avec $0 \leq s_{1}<\cdots<s_{j} \leq n-1$.

On rappelle que $\mathcal{H}^{*} V_{n}$ désigne la cohomologie $H^{*} V_{n}$ munie de l'action tordue de $\mathrm{GL}_{n}$ donnée par $g * f=\operatorname{det}(g)^{\frac{p-1}{2}}(g \cdot f)$. On déduit du théorème 6.3 et du lemme 6.1 le 
Corollaire 6.4. - $\left(\mathcal{H}^{*} V_{n}\right)^{\mathrm{GL}_{n}}$ est un $\mathbb{F}_{p}\left[y_{1}, \ldots, y_{n}\right]^{\mathrm{GL}_{s}}$-module libre de base

$$
\left\{\mathbf{L}_{n}^{\frac{p-1}{2}}, \quad \mathbf{M}_{n ; s_{1}, \ldots, s_{j}} \mathbf{L}_{n}^{\frac{p-3}{2}}\right\}
$$

avec $0 \leq s_{1}<\cdots<s_{j} \leq n-1$.

6.2. Structure de $D(n)$ et $\mathfrak{D}(n)$. - Soit $G$ un groupe fini et soit $S$ un sousgroupe du groupe symétrique $\Sigma_{k}$. Le produit en couronne $S \int G$ est défini comme étant le produit semi-direct

$$
G^{k} \hookrightarrow S \ltimes G^{k} \rightarrow S .
$$

La multiplication de $S \ltimes G^{k}$ est donné par

$$
\left(s ; g_{1}, \ldots, g_{k}\right)\left(s^{\prime} ; g_{1}^{\prime}, \ldots, g_{k}^{\prime}\right)=\left(s s^{\prime} ; g_{1} g_{s^{-1}(1)}^{\prime}, \ldots, g_{k} g_{s^{-1}(k)}^{\prime}\right)
$$

pour $\left(g_{1}, \ldots, g_{k}\right),\left(g_{1}^{\prime}, \ldots, g_{k}^{\prime}\right) \in G^{k}$ et $s, s^{\prime} \in S$. Si $G$ est un sous-groupe du groupe symétrique $\Sigma_{m}$, alors $S \int G$ agit sur les couples $(i, j), 1 \leq i \leq k, 1 \leq$ $j \leq m$ :

$$
\left(s ; g_{1}, \ldots, g_{k}\right) \cdot(i, j)=\left(s(i), g_{i}(j)\right)
$$

pour $\left(s ; g_{1}, \ldots, g_{k}\right) \in S \int G$. Le groupe $S \int G$ est ainsi un sous-groupe de $\Sigma_{k m}$.

Soient maintenant $\left(a_{1}, \ldots, a_{n}\right)$ la base canonique de $V_{n}$ et $A_{i}$ le sous-espace engendré par $a_{i}, 1 \leq i \leq n$. On a $A_{i} \subset \Sigma_{p}$. On désigne par $\Sigma_{p^{n}, p}$ le produit en couronne itéré

$$
\left(\cdots\left(A_{1} \int A_{2}\right) \int A_{3} \cdots\right) \int A_{n} .
$$

Il est bien connu que $\Sigma_{p^{n}, p}$ est un sous-groupe de Sylow de $\Sigma_{p^{n}}$ contenant $V_{n}$.

Si $K$ est un sous-groupe de $G$ et $M$ un $G$-module, on note $i(K, G ; M)$ le morphisme $H^{*}(G ; M) \rightarrow H^{*}(K ; M)$ induit par l'inclusion $K \hookrightarrow G$. Si $M$ est le $G$-module trivial, $i(K, G ; M)$ se note simplement $i(K, G)$.

Par définition, $D(n)=\operatorname{Im} i\left(V_{n}, \Sigma_{p^{n}}\right)$ et $\mathfrak{D}(n)=\operatorname{Im} i\left(V_{n}, \Sigma_{p^{n}} ; Z / p\right)$.

La structure de $D(n)$ en tant que $\mathbb{F}_{p}$-algèbre a été déterminée pour la première fois par H. Mui [18]. La méthode de calcul de $\operatorname{Im} i\left(V_{n}, \Sigma_{p^{n}}\right)$ de [18] s'appuie sur la théorie d'invariants modulaires de $\mathrm{GL}_{n}$ et le résultat suivant.

THÉORÈme 6.5 ([18]). - Soit $G$ un sous-groupe de $\Sigma_{p^{n}}$ contenant $\Sigma_{p^{n}, p}$. Alors

$$
\operatorname{Im} i\left(V_{n}, G ; M\right)=\operatorname{Im} i\left(V_{n}, \Sigma_{p^{n}, p} ; M\right) \cap H^{*}\left(V_{n} ; M\right)^{W_{G}\left(V_{n}\right)}
$$

où $W_{G}\left(V_{n}\right)$ est le groupe de Weyl de $V_{n}$ dans $G$.

Démonstration. - Voir la démonstration de [18, I.1.4]. La démonstration repose sur la formule des doubles classes $[5,2.4 .5]$ et le fait que $V_{n} \subset \Sigma_{p^{n}, p} \subset G$ est un système fermé, i.e. si un sous-groupe de $\Sigma_{p^{n}, p}$ est conjugué à $V_{n}$ dans $G$, alors il est conjugué à $V_{n}$ dans $\Sigma_{p^{n}, p}[18$, I.2.1]. 
Posons $\mathcal{M}_{n}(p):=\operatorname{Im} i\left(V_{n}, \Sigma_{p^{n}, p}\right)$. Cette algèbre a été aussi explicitée par Mui.

THÉORÈme 6.6 ([18]). — On a

$$
\mathcal{M}_{n}(p)=\Lambda\left(\mathbf{U}_{1}, \ldots, \mathbf{U}_{n}\right) \otimes \mathbb{F}_{p}\left[\mathbf{V}_{1}, \ldots, \mathbf{V}_{n}\right]
$$

avec $\mathbf{U}_{k}=\mathbf{M}_{k ; k-1} \mathbf{L}_{k-1}^{\frac{p-3}{2}}, 1 \leq k \leq n$.

Notons que, d'après [18],

$$
\mathbb{F}_{p}\left[y_{1}, \ldots, y_{n}\right]^{\mathrm{U}_{n}}=\mathbb{F}_{p}\left[\mathbf{V}_{1}, \ldots, \mathbf{V}_{n}\right]
$$

où $\mathrm{U}_{n}$ désigne le sous-groupe de Sylow de $\mathrm{GL}_{n}$ des matrices triangulaires supérieures avec 1 sur la diagonale. Le groupe $\mathrm{U}_{n}$ est aussi le groupe de Weyl de $V_{n}$ dans $\Sigma_{p^{n}, p}$, d'où $\mathcal{M}_{n}(p)$ est une sous-algèbre de $\left(H^{*} V_{n}\right)^{\mathrm{U}_{n}}$.

On note $\mathfrak{e}_{n}=\mathbf{L}_{n}^{\frac{p-1}{2}}$ et $\widetilde{\mathbf{M}}_{n ; s}=\mathbf{M}_{n ; s} \mathbf{L}_{n}^{\frac{p-3}{2}}$. Le degré de $\mathfrak{e}_{n}$ est $p^{n}-1$ et le degré de $\widetilde{\mathbf{M}}_{n ; s}$ est $p^{n}-2 p^{s}$.

Les trois lemmes suivants seront utiles pour le calcul de l'intersection de $\mathcal{M}_{n}(p)$ et l'algèbre $\left(H^{*} V_{n}\right)^{G}$ avec $G$ un sous-groupe de $\mathrm{GL}_{n}$.

Lemme 6.7. - Pour $0 \leq s<k \leq n$, on a $\mathbf{Q}_{k, s}, \mathfrak{e}_{s}, \widetilde{\mathbf{M}}_{k, s} \in \mathcal{M}_{n}(p)$. De plus si $f$ est un élément de $\Lambda\left(x_{1}, \ldots, x_{n}\right) \otimes \mathbb{F}_{p}\left[y_{1}, \ldots, y_{n}\right]$ tel que $f \cdot \mathbf{V}_{n} \in \mathcal{M}_{n}(p)$, alors $f \in \mathcal{M}_{n}(p)$.

Démonstration. — On répète la démonstration de II.6.3 et II.6.4 dans [18] pour le premier énoncé. Comme $\mathbf{Q}_{k, s}$ et $\mathfrak{e}_{s}$ sont des $\mathrm{U}_{n}$-invariants de $\mathbb{F}_{p}\left[y_{1}, \ldots, y_{n}\right]$, il est clair que $\mathbf{Q}_{k, s}$ et $\mathfrak{e}_{s}$ appartiennent à $\mathcal{M}_{n}(p)$.

Ensuite, on a $\widetilde{\mathbf{M}}_{k, k-1}=\mathbf{U}_{k} \mathbf{V}_{k}^{\frac{p-3}{2}}$, d'où $\widetilde{\mathbf{M}}_{k, k-1} \in \mathcal{M}_{n}(p)$. Pour $s \leq k-2$, utilisant la relation $\mathbf{M}_{k, s}=\mathbf{M}_{k-1, s} \mathbf{V}_{k}+\mathbf{M}_{k, k-1} \mathbf{Q}_{k-1, s}$, on a

$$
\widetilde{\mathbf{M}}_{k, s}=\widetilde{\mathbf{M}}_{k-1, s} \mathbf{V}_{k}^{\frac{p-1}{2}}+\mathbf{U}_{k} \mathbf{V}_{k}^{\frac{p-3}{2}} \mathbf{Q}_{k-1, s}
$$

Comme $\mathbf{U}_{k} \mathbf{V}_{k}^{\frac{p-3}{2}} \mathbf{Q}_{k-1, s} \in \mathcal{M}_{n}(p)$, il est clair que $\widetilde{\mathbf{M}}_{k, s} \in \mathcal{M}_{n}(p)$ par récurrence.

Pour le dernier énoncé, on pose $\mathcal{M}_{n}^{\prime}(p)=\Lambda\left(\mathbf{U}_{1}, \ldots, \mathbf{U}_{n}\right) \otimes \mathbb{F}_{p}\left[\mathbf{V}_{1}, \ldots, \mathbf{V}_{n-1}\right]$. Comme $f \cdot \mathbf{V}_{n} \in \mathcal{M}_{n}(p)$, on peut écrire $f \cdot \mathbf{V}_{n}=f_{0}+f_{1}$ avec $f_{0} \in \mathcal{M}_{n}^{\prime}(p)$ et $f_{1} \in$ $\mathcal{M}_{n}(p) \cdot \mathbf{V}_{n}$. Si $f_{0}$ est non-nul, alors $f_{0}$ est divisible par $y_{n}$. Or, aucun élément non-nul de $\mathcal{M}_{n}^{\prime}(p)$ n'est divisible par $y_{n}$ car, d'une part, les $\mathbf{U}_{1}, \ldots, \mathbf{U}_{n-1}$ et $\mathbf{V}_{1}, \ldots, \mathbf{V}_{n-1}$ ne contiennent pas $x_{n}$ et $y_{n}$, et d'autre part,

$$
\left.\mathbf{U}_{n}\right|_{y_{n}=0}=(-1)^{n} x_{n} \mathfrak{e}_{n-1} \neq 0 .
$$

Il s'ensuit $f_{0}=0$, donc $f \in \mathcal{M}_{n}(p)$. Le lemme est démontré. 
Lemme 6.8. - Soit $f \in \Lambda^{k}\left(x_{1}, \ldots, x_{n}\right) \otimes \mathbb{F}_{p}\left[y_{1}, \ldots, y_{n}\right]$ de la forme

$$
f=\sum_{I} x_{I} f_{I}\left(y_{1}, \ldots, y_{n}\right) \text {. }
$$

Si $f$ est un élément de $\mathcal{M}_{n}(p)$, alors $y_{1}^{\frac{(p-1)(k-1)}{2}}$ divise $f_{I_{0}}$ avec $I_{0}=\{1, \ldots, k\}$.

Démonstration. - Cette remarque apparaît dans la démonstration de II.6.6 de [18]. Comme $f \in \mathcal{M}_{n}(p), f$ est aussi de la forme

$$
f=\sum_{I} \mathbf{U}_{I} g_{I}\left(\mathbf{V}_{1}, \ldots, \mathbf{V}_{n}\right) .
$$

Un calcul direct donne $\mathbf{U}_{1} \cdots \mathbf{U}_{k}=(-1)^{\left(\begin{array}{c}k \\ 2\end{array}\right)} x_{1} \cdots x_{k} \mathbf{L}_{1}^{\frac{p-1}{2}} \cdots \mathbf{L}_{k-1}^{\frac{p-1}{2}}$ qui est divisible par $y_{1}^{\frac{(p-1)(k-1)}{2}}$. Le lemme suit.

Lemme 6.9 ([18, I.4.15]). - Soient $1 \leq d \leq p-1$ et $f \in \Lambda^{k}\left(x_{1}, \ldots, x_{n}\right) \otimes$ $\mathbb{F}_{p}\left[y_{1}, \ldots, y_{n}\right]$ un invariant par rapport à $\operatorname{det}^{d}$ de la forme

$$
f=\sum_{I} x_{I} f_{I}\left(y_{1}, \ldots, y_{n}\right)
$$

avec $I$ une partie ordonnée de cardinal $k$ de $\{1, \ldots, n\}$. Supposons que $y_{i}^{m}$ divise $f_{I}$ pour certain $i$ et certain $I$, alors $\mathbf{L}_{n}^{m+\overline{d-1-m}}$ divise $f_{I}$ pour tout $I$. Ici $a \equiv \bar{a}$ $\bmod p-1$ avec $0 \leq \bar{a} \leq p-2$.

On est maintenant en mesure de décrire la structure de $D(n)$ et $\mathfrak{D}(n)$. Pour les calculs dans les sections qui suivent, il nous suffit de comprendre la structure de $D(n)$ et $\mathfrak{D}(n)$ en tant que $\mathbb{F}_{p}$-espaces vectoriels.

ThÉoRÈme 6.10 ([18]). - D $D(n)$ est un $\mathbb{F}_{p}\left[\mathbf{Q}_{n, 0}, \ldots, \mathbf{Q}_{n, n-1}\right]$-module libre de base

$$
\left\{1, \quad \mathbf{M}_{n ; s_{1}, \ldots, s_{j}} \mathbf{L}_{n}^{p-2+(p-1)\left[\frac{j-1}{2}\right]}\right\}
$$

avec $0 \leq s_{1}<\cdots<s_{j} \leq n-1$.

Démonstration. - On a $D(n)=\mathcal{M}_{n}(p) \cap\left(H^{*} V_{n}\right)^{\mathrm{GL}_{n}}$. Posons $j=2 j^{\prime}+e$, $e=0,1$. Comme

$$
\begin{aligned}
\mathbf{M}_{n ; s_{1}, \ldots, s_{j}} \mathbf{L}_{n}^{p-2+(p-1)\left[\frac{j-1}{2}\right]} & =(-1)^{\left(\begin{array}{l}
j \\
2
\end{array}\right)} \widetilde{\mathbf{M}}_{n, s_{1}} \cdots \widetilde{\mathbf{M}}_{n, s_{j}} \mathbf{L}_{n}^{p-1+(p-1)\left[\frac{j-1}{2}\right]-\frac{(p-1) j}{2}} \\
& =(-1)^{\left(\begin{array}{l}
j \\
2
\end{array}\right)} \widetilde{\mathbf{M}}_{n, s_{1}} \cdots \widetilde{\mathbf{M}}_{n, s_{j}} \mathfrak{e}_{n}^{e}
\end{aligned}
$$

on déduit du lemme 6.7 et du théorème 6.3 que le module donné dans le théorème est contenu dans $\mathcal{M}_{n}(p) \cap H^{*} V_{n}^{\mathrm{GL}}$.

TOME $140-2012-\mathrm{N}^{\mathrm{O}} 2$ 
Montrons l'inclusion inverse. Soit $f \in \Lambda^{j}\left(x_{1}, \ldots, x_{n}\right) \otimes \mathbb{F}_{p}\left[y_{1}, \ldots, y_{n}\right]$ un élément de $\mathcal{M}_{n}(p) \cap H^{*} V_{n}^{\mathrm{GL}_{n}}$ de la forme

$$
f=\sum_{I} x_{I} f_{I}\left(y_{1}, \ldots, y_{n}\right) .
$$

Si $j=0$, alors $f$ est un élément de $\mathbb{F}_{p}\left[\mathbf{Q}_{n, 0}, \ldots, \mathbf{Q}_{n, n-1}\right]$. Supposons que $j>0$. D'après le lemme $6.8, y_{1}^{\frac{(p-1)(j-1)}{2}}$ divise $f_{I_{0}}$ avec $I_{0}=\{1, \ldots, j\}$. Comme $f$ est $\mathrm{GL}_{n}$-invariant, i.e. invariant par rapport à $\operatorname{det}^{p-1}$, on déduit du lemme 6.9 que $\mathbf{L}_{n}^{p-2+(p-1)\left[\frac{j-1}{2}\right]}$ divise $f$ car

$$
\frac{(p-1)(j-1)}{2}+\overline{p-2-\frac{(p-1)(j-1)}{2}}=p-2+(p-1)\left[\frac{j-1}{2}\right] .
$$

Utilisant le théorème $6.3, f$ est un élément du module donné dans le théorème.

ThÉORÈme 6.11 ([24]). - $\mathfrak{D}(n)$ est un $\mathbb{F}_{p}\left[\mathbf{Q}_{n, 0}, \ldots, \mathbf{Q}_{n, n-1}\right]$-module libre de base

$$
\left\{\mathbf{L}_{n}^{\frac{p-1}{2}}, \quad \mathbf{M}_{n ; s_{1}, \ldots, s_{j}} \mathbf{L}_{n}^{\frac{p-3}{2}+(p-1)\left[\frac{j}{2}\right]}\right\}
$$

avec $0 \leq s_{1}<\cdots<s_{j} \leq n-1$.

Démonstration. - On a $\mathfrak{D}(n)=\mathcal{M}_{n}(p) \cap\left(\mathcal{H}^{*} V_{n}\right)^{\mathrm{GL}_{n}}$. Posons $j=2 j^{\prime}+e$, $e=0,1$. Comme

$$
\begin{aligned}
\mathbf{M}_{n ; s_{1}, \ldots, s_{j}} \mathbf{L}_{n^{\frac{p-3}{2}}+(p-1)\left[\frac{j}{2}\right]} & =(-1)^{\left(\begin{array}{l}
j \\
2
\end{array}\right)} \widetilde{\mathbf{M}}_{n, s_{1}} \cdots \widetilde{\mathbf{M}}_{n, s_{j}} \mathbf{L}_{n}^{\frac{p-1}{2}+(p-1)\left[\frac{j}{2}\right]-\frac{(p-1) j}{2}} \\
& =(-1)^{\left(\begin{array}{l}
j \\
2
\end{array}\right)} \widetilde{\mathbf{M}}_{n, s_{1}} \cdots \widetilde{\mathbf{M}}_{n, s_{j}} \mathfrak{e}_{n}^{1-e},
\end{aligned}
$$

on déduit du lemme 6.7 et du corollaire 6.4 que le module donné dans le théorème est contenu dans $\mathcal{M}_{n}(p) \cap\left(\mathcal{H}^{*} V_{n}\right)^{\mathrm{GL}_{n}}$.

Montrons l'inclusion inverse. Soit $f \in \Lambda^{j}\left(x_{1}, \ldots, x_{n}\right) \otimes \mathbb{F}_{p}\left[y_{1}, \ldots, y_{n}\right]$ un élément de $\mathcal{M}_{n}(p) \cap\left(\mathcal{H}^{*} V_{n}\right)^{\mathrm{GL}_{n}}$ de la forme

$$
f=\sum_{I} x_{I} f_{I}\left(y_{1}, \ldots, y_{n}\right) \text {. }
$$

Si $j=0$, alors $f$ est un élément de $\mathbb{F}_{p}\left[\mathbf{Q}_{n, 0}, \ldots, \mathbf{Q}_{n, n-1}\right] \mathbf{L}_{n}^{\frac{p-1}{2}}$. Supposons $j>0$. D'après le lemme $6.8, y_{1}^{\frac{(p-1)(j-1)}{2}}$ divise $f_{I_{0}}$ avec $I_{0}=\{1, \ldots, j\}$. Comme $f$ est invariant par rapport à det ${ }^{\frac{p-1}{2}}$, on déduit du lemme 6.9 que $\mathbf{L}_{n^{\frac{p-3}{2}}+(p-1)\left[\frac{j}{2}\right]}$ divise $f$ car

$$
\frac{(p-1)(j-1)}{2}+\overline{\frac{p-3}{2}-\frac{(p-1)(j-1)}{2}}=\frac{p-3}{2}+(p-1)\left[\frac{j}{2}\right] .
$$

Utilisant le théorème $6.3, f$ est un élément du module donné dans le théorème. 
Corollaire 6.12 ([24]). - On a

$$
D(n) \oplus \mathfrak{D}(n) \cong \Lambda\left(\widetilde{\mathbf{M}}_{n, 0}, \ldots, \widetilde{\mathbf{M}}_{n, n-1}\right) \otimes \mathbb{F}_{p}\left[\mathfrak{e}_{n}, \mathbf{Q}_{n, 1}, \ldots, \mathbf{Q}_{n, n-1}\right]
$$

REMARQUe 6.13. - On note $\mathscr{A}_{p^{n}}$ le sous-groupe alterné du groupe symétrique $\Sigma_{p^{n}}$. D'après le lemme d'Eckmann-Shapiro, on a $H^{*} \mathscr{A}_{p^{n}} \cong$ $H^{*}\left(\Sigma_{p^{n}} ; \mathbb{1}_{\mathscr{A}_{p^{n}}}^{\Sigma_{p^{n}}}\right)$. On vérifie que le module induit $\mathbb{1}_{\mathscr{A}_{p^{n}}}^{\Sigma_{p^{n}}}$ est isomorphe à la somme directe de la représentation triviale $\mathbb{Z} / p$ et la représentation signature $Z / p$. Il s'ensuit que

$$
D(n) \oplus \mathfrak{D}(n) \cong \operatorname{Im}\left(H^{*} \mathscr{A}_{p^{n}} \stackrel{i\left(V_{n}, \mathscr{A}_{p^{n}}\right)}{\longrightarrow} H^{*} V_{n}\right) .
$$

Le corollaire 6.12 alors peut être trouvé dans [19].

6.3. Calcul de $\left(D(n): L_{1}\right)$ u — Soit $\mathrm{G}_{n-1}\left(\right.$ resp. $\left.\mathrm{GL}_{n-1}\right)$ le sous-groupe de $\mathrm{GL}_{n}$ des matrices de la forme

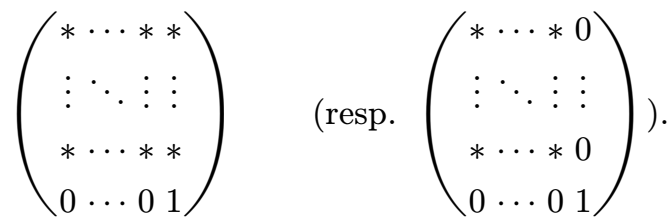

Soit $\mathrm{T}: \mathcal{U} \rightarrow \mathcal{U}$ le foncteur de Lannes [12]. On a

$$
\mathrm{T}\left(\left(H^{*} V_{n}\right)^{\mathrm{GL}_{n}}\right)=\operatorname{Map}_{\mathrm{GL}_{n}}\left(V_{n}, H^{*} V_{n}\right) .
$$

Rappelons que si $\left(a_{1}, \ldots, a_{n}\right)$ est la base canonique de $V_{n}$, alors l'action à droite de $\mathrm{GL}_{n}$ sur $V_{n}$ est donnée par $\left(a_{1} \cdot g, \ldots, a_{n} \cdot g\right)=\left(a_{1}, \ldots, a_{n}\right) g^{t}$, le produit matriciel de $\left(a_{1}, \ldots, a_{n}\right)$ par le transposé de $g \in \mathrm{GL}_{n}$. L'espace vectoriel $V_{n}$ muni de cette action de $\mathrm{GL}_{n}$ se décompose en deux orbites : celle de 0 et celle de $a_{n}$. On a évidemment que le fixateur dans $\mathrm{GL}_{n}$ de 0 (resp. de $a_{n}$ ) est $\mathrm{GL}_{n}$ (resp. $\mathrm{G}_{n-1}$ ). On en déduit que

$$
\overline{\mathrm{T}}\left(\left(H^{*} V_{n}\right)^{\mathrm{GL}_{n}}\right)=\left(H^{*} V_{n}\right)^{\mathrm{G}_{n-1}},
$$

où $\overline{\mathrm{T}}$ désigne le foncteur de Lannes réduit.

THÉORÈme 6.14. - $\left(H^{*} V_{n}\right)^{\mathrm{G}_{n-1}}$ est un $\mathbb{F}_{p}\left[\mathbf{Q}_{n-1,0}, \ldots, \mathbf{Q}_{n-1, n-2}, \mathbf{V}_{n}\right]-m o-$ dule libre de base

$$
\left\{1, \quad \mathbf{M}_{n-1 ; s_{1}, \ldots, s_{i}} \mathbf{L}_{n-1}^{p-2}, \quad \mathbf{M}_{n ; n-1} \mathbf{L}_{n-1}^{p-2}, \quad \mathbf{M}_{n ; r_{1}, \ldots, r_{j-1}, n-1} \mathbf{L}_{n-1}^{p-2}\right\}
$$

où $0 \leq s_{1}<\cdots<s_{i} \leq n-2$ et $0 \leq r_{1}<\cdots<r_{j-1} \leq n-2$. 
Démonstration. - Observons d'abord que

$$
\mathbb{F}_{p}\left[y_{1}, \ldots, y_{n}\right]^{\mathbf{G}_{n-1}}=\mathbb{F}_{p}\left[\mathbf{Q}_{n-1,0}, \ldots, \mathbf{Q}_{n-1, n-2}, \mathbf{V}_{n}\right] .
$$

Donc il est clair que le module libre donné dans le théorème est contenu dans $\left(H^{*} V_{n}\right)^{\mathrm{G}_{n-1}}$.

Montrons l'inclusion inverse. On note $\mathrm{S}_{n-1}$ le sous-groupe $\mathrm{SL}_{n}$ des matrices de la forme

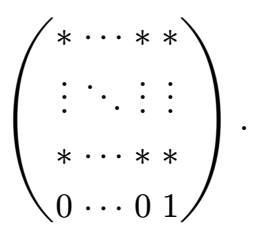

D'après [16], $\left(H^{*} V_{n}\right)^{\mathrm{S}_{n-1}}$ est un $\mathbb{F}_{p}\left[\mathbf{L}_{n-1}, \mathbf{Q}_{n-1,1}, \ldots, \mathbf{Q}_{n-1, n-2}, \mathbf{V}_{n}\right]$-module libre de base

$$
\left\{1, \quad \mathbf{M}_{n-1 ; s_{1}, \ldots, s_{i}}, \quad \mathbf{M}_{n ; n-1}, \quad \mathbf{M}_{n ; r_{1}, \ldots, r_{j-1}, n-1}\right\}
$$

où $0 \leq s_{1}<\cdots<s_{i} \leq n-2$ et $0 \leq r_{1}<\cdots<r_{j-1} \leq n-2$.

Soit $f$ un élément de $\left(H^{*} V_{n}\right)^{\mathrm{G}_{n-1}}, f \in \Lambda^{j}\left(x_{1}, \ldots, x_{n}\right) \otimes \mathbb{F}_{p}\left[y_{1}, \ldots, y_{n}\right]$. Si $j=0$, alors $f \in \mathbb{F}_{p}\left[\mathbf{Q}_{n-1,0}, \ldots, \mathbf{Q}_{n-1, n-2}, \mathbf{V}_{n}\right]$. Supposons que $j>0$. Comme $\mathrm{S}_{n-1} \subset \mathrm{G}_{n-1}, f$ s'écrit

$$
f=\sum_{S} \mathbf{M}_{n-1 ; s_{1}, \ldots, s_{j}} f_{S}+\sum_{R} \mathbf{M}_{n ; r_{1}, \ldots, r_{j-1}, n-1} f_{R}
$$

avec $f_{S}, f_{R} \in \mathbb{F}_{p}\left[\mathbf{L}_{n-1}, \mathbf{Q}_{n-1,1}, \ldots, \mathbf{Q}_{n-1, n-2}, \mathbf{V}_{n}\right]$. Comme $f$ est $\mathrm{GL}_{n-1}$-invariant, on déduit du lemme 6.1 que $f_{S}$ et $f_{R}$ sont divisibles par $\mathbf{L}_{n-1}^{p-2}$. Il s'ensuit que $f$ est un élément du module libre donné dans le théorème.

Considérons ensuite le calcul de $\mathrm{T}(D(n))$. Rappelons que, contrairement au cas $p=2, D(n)$ est un sous-module propre de $\left(H^{*} V_{n}\right)^{\mathrm{GL}_{n}}$. A cause de cela, on va calculer $\mathrm{T}(D(n))$ en utilisant la définition $D(n)=\operatorname{Im} i\left(V_{n}, \Sigma_{p^{n}}\right)$.

Soit $\mathscr{C}_{n}$ le centralisateur dans le groupe $\Sigma_{p^{n}}$ du vecteur de base $a_{n} \in V_{n}$ :

$$
\mathscr{C}_{n}=\left\{f: V_{n} \rightarrow V_{n} \mid f\left(v+a_{n}\right)=f(v)+a_{n}, \forall v \in V_{n}\right\} \subset \Sigma_{p^{n}} .
$$

Proposition 6.15. - On a $\overline{\mathrm{T}}\left(\operatorname{Im} i\left(V_{n}, \Sigma_{p^{n}}\right)\right) \cong \operatorname{Im} i\left(V_{n}, \mathscr{C}_{n}\right)$.

Démonstration. - Rappelons d'abord la formule de l'image de T sur la cohomologie d'un groupe fini. Soit $G$ un groupe fini, $\mathrm{T}\left(H^{*} G\right)$ est donné par ([12]) :

$$
\mathrm{T}\left(H^{*} G\right) \cong \prod_{\varphi \in \operatorname{Rep}(\mathbb{Z} / p, G)} H^{*} G_{\varphi} .
$$

Ici $\operatorname{Rep}(\mathbb{Z} / p, G)$ désigne le quotient de l'action par conjugaison de $G$ sur $\operatorname{Hom}(\mathbb{Z} / p, G)$ et $G_{\varphi}$ le centralisateur dans $G$ de l'image de $\varphi$. Le morphisme 
$\mathrm{T}\left(H^{*} G\right) \rightarrow H^{*} G_{\varphi}$ est induit par le morphisme évident $\mathbb{Z} / p \times G_{\varphi} \rightarrow G$, $(x, g) \mapsto \varphi(x) g$.

Maintenant, soit $f: G^{\prime} \rightarrow G$ un homomorphisme de groupe. Par fontorialité, le morphisme $\mathrm{T}\left(f^{*}\right): \mathrm{T}\left(H^{*} G\right) \rightarrow \mathrm{T}\left(H^{*} G^{\prime}\right)$ est le produit des morphismes

$$
\mathrm{T}\left(f_{\varphi}^{*}\right): H^{*} G_{\varphi} \rightarrow \prod_{\substack{\varphi^{\prime} \in \operatorname{Rep}\left(\mathbb{Z} / p, G^{\prime}\right) \\ \varphi=f \circ \varphi^{\prime}}} H^{*} G_{\varphi^{\prime}}^{\prime}
$$

$\varphi$ décrivant $\operatorname{Rep}(\mathbb{Z} / p, G)$. On en déduit que $\mathrm{T}\left(\operatorname{Im} f^{*}\right)$ est l'image du morphisme

$$
\prod_{\varphi \in \operatorname{Im} \operatorname{Rep}(f)} H^{*} G_{\varphi} \stackrel{\prod_{\varphi \in \operatorname{Im} \operatorname{Rep}(f)} \mathrm{T}\left(f_{\varphi}^{*}\right)}{\longrightarrow} \prod_{\varphi \in \operatorname{Im} \operatorname{Rep}(f)}\left(\prod_{\varphi^{\prime} \in \operatorname{Rep}(f)^{-1}(\varphi)} H^{*} G_{\varphi^{\prime}}^{\prime}\right),
$$

où $\operatorname{Rep}(f): \operatorname{Rep}\left(\mathbb{Z} / p, G^{\prime}\right) \rightarrow \operatorname{Rep}(\mathbb{Z} / p, G)$ est l'application induite par $f: G^{\prime} \rightarrow$ $G$.

Revenons au cas où $f$ est le plongement naturel $i_{n}: V_{n} \rightarrow \Sigma_{p^{n}}$. On a

$$
\operatorname{Rep}\left(\mathbb{Z} / p, V_{n}\right)=V_{n} .
$$

Considérons l'image de $\operatorname{Rep}\left(i_{n}\right): \operatorname{Rep}\left(\mathbb{Z} / p, V_{n}\right) \rightarrow \operatorname{Rep}\left(\mathbb{Z} / p, \Sigma_{p^{n}}\right)$. On a évidemment que $\operatorname{Rep}\left(i_{n}\right)(0)=0$. Si $v_{1}$ et $v_{2}$ sont deux éléments non-nuls de $V_{n}=\operatorname{Rep}\left(\mathbb{Z} / p, V_{n}\right)$, alors $v_{1}$ est conjugué à $v_{2}$ dans $\Sigma_{p^{s}}$ par un automorphisme $f \in \operatorname{Aut}\left(V_{n}\right) \subset \Sigma_{p^{n}}$ qui envoie $v_{1}$ à $v_{2}$. En effet, pour tout $v \in V_{n}$, on a

$$
\left(f \circ v_{1} \circ f^{-1}\right)(v)=f\left(v_{1}+f^{-1}(v)\right)=f\left(v_{1}\right)+v=v_{2}+v=v_{2}(v),
$$

i.e. $f \circ v_{1} \circ f^{-1}=v_{2}$.

On en déduit que $\operatorname{Im} \operatorname{Rep}\left(i_{n}\right)=V_{n} / \mathrm{GL}_{n}$ et celui-ci est représentée par 0 et $a_{n} \in V_{n} \subset \Sigma_{p_{n}},\left\{a_{1}, \ldots, a_{n}\right\}$ étant la base canonique de $V_{n}$. Le centralisateur dans $\Sigma_{p^{n}}$ de $0\left(\operatorname{resp} . a_{n}\right)$ est alors $\Sigma_{p^{n}}\left(\operatorname{resp} . \mathscr{C}_{n}\right)$.

Soit $j_{n}: V_{n} \hookrightarrow \mathscr{C}_{n}$ l'inclusion naturelle. Par ce qui précède, $\mathrm{T}\left(\operatorname{Im} i\left(V_{n}, \Sigma_{p^{n}}\right)\right)$ est l'image du morphisme :

$$
H^{*} \Sigma_{p^{n}} \times H^{*} \mathscr{C}_{n} \stackrel{i\left(V_{n}, \Sigma_{p^{n}}\right) \times \prod_{v \in V_{n} \backslash\{0\}} i\left(V_{n}, \mathscr{C}_{n}\right)}{\longrightarrow} H^{*} V_{n} \times \prod_{v \in V_{n} \backslash\{0\}} H^{*} V_{n}
$$

Il suit que $\overline{\mathrm{T}}\left(\operatorname{Im} i\left(V_{n}, \Sigma_{p^{n}}\right)\right) \cong \operatorname{Im} i\left(V_{n}, \mathscr{C}_{n}\right)$. La proposition est démontrée.

Rappelons que $\mathrm{G}_{n-1}$ est le sous-groupe de $\mathrm{GL}_{n}$ des matrices de la forme

$$
\left(\begin{array}{cccc}
* & \cdots & * & * \\
\vdots & \ddots & \vdots & \vdots \\
* & \cdots & * & * \\
0 & \cdots & 0 & 1
\end{array}\right) .
$$

TOME $140-2012-\mathrm{N}^{\mathrm{O}} 2$ 
Corollaire 6.16. - On a $\overline{\mathrm{T}}\left(\operatorname{Im} i\left(V_{n}, \Sigma_{p^{n}}\right)\right) \cong \operatorname{Im} i\left(V_{n}, \Sigma_{p^{n}, p}\right) \cap\left(H^{*} V_{n}\right)^{\mathrm{G}_{n-1}}$.

Démonstration. - Montrons d'abord que $V_{n} \subset \Sigma_{p^{n}, p} \subset \mathscr{C}_{n}$. On considère $\Sigma_{p^{n-1}}$ le groupe des permutations (de l'ensemble des points) de $V_{n-1}$, le sousespace de $V_{n}$ engendré par $a_{1}, \ldots, a_{n-1}$. Soit $i_{n-1}: V_{n-1} \hookrightarrow \Sigma_{p^{n-1}}$ l'inclusion naturelle. Soit $A_{n}$ le sous-espace de $V_{n}$ engendré par $a_{n}$. Vérifions que $\Sigma_{p^{n-1}} \int A_{n}$ est un sous-groupe de $\mathscr{C}_{n}$. Soit $f$ un élément de $\Sigma_{p^{n-1}} \int A_{n}$ de la forme

$$
f=\left(s ; \lambda_{1} a_{n}, \ldots, \lambda_{p^{n-1}} a_{n}\right)
$$

avec $s \in \Sigma_{p^{n-1}}$ et $\lambda_{1} a_{n}, \ldots, \lambda_{p^{n-1}} a_{n} \in A_{n}$. Par définition du produit en couronne, on a

$$
f\left(v+\lambda a_{n}\right)=s(v)+\lambda_{i_{n-1}(v)} a_{n}+\lambda a_{n}
$$

pour $v \in V_{n-1}$ et $\lambda a_{n} \in A_{n}$. D'autre part, on peut réécrire le groupe $\mathscr{C}_{n}$ comme suit :

$$
\mathscr{C}_{n}=\left\{f: V_{n} \rightarrow V_{n} \mid f\left(v+\lambda a_{n}\right)=f(v)+\lambda a_{n}, \forall v \in V_{n-1}, \lambda \in \mathbb{F}_{p}\right\} .
$$

On en déduit que $f$ est un élément de $\mathscr{C}_{n}$, d'où $\Sigma_{p^{n-1}} \int A_{n} \subset \mathscr{C}_{n}$. Comme $\Sigma_{p^{n}, p}$ est un sous-groupe de $\Sigma_{p^{n-1}} \int A_{n}$ contenant $V_{n}$, on a $V_{n} \subset \Sigma_{p^{n}, p} \subset \mathscr{C}_{n}$.

Montrons maintenant le corollaire. D'après le théorème 6.5 , on a

$$
\operatorname{Im} i\left(V_{n}, \mathscr{C}_{n}\right) \cong \operatorname{Im} i\left(V_{n}, \Sigma_{p^{n}, p}\right) \cap\left(H^{*} V_{n}\right)^{W \mathscr{C}_{n}\left(V_{n}\right)}
$$

D'autre part, d'après [10, Proposition 3.5], le groupe de Weyl $W_{\mathscr{C}_{n}}\left(V_{n}\right)$ s'identifie à $\mathrm{GL}_{n} \cap \mathscr{C}_{n}$. Par définition, on a

$$
\begin{aligned}
\mathrm{GL}_{n} \cap \mathscr{C}_{n} & =\left\{f \in \operatorname{Aut}\left(V_{n}\right) \mid f\left(v+a_{n}\right)=f(v)+a_{n}, \forall v \in V_{n}\right\} \\
& =\left\{f \in \operatorname{Aut}\left(V_{n}\right) \mid f\left(a_{n}\right)=a_{n}\right\} \\
& =\mathrm{G}_{n-1} .
\end{aligned}
$$

Le corollaire suit.

THÉORÈme 6.17. - $\overline{\mathrm{T}}(D(n))$ est un $\mathbb{F}_{p}\left[\mathbf{Q}_{n-1,0}, \ldots, \mathbf{Q}_{n-1, n-2}, \mathbf{V}_{n}\right]$-module libre de base

$$
\begin{aligned}
& \left\{1, \mathbf{M}_{n-1 ; s_{1}, \ldots, s_{i}} \mathbf{L}_{n-1}^{p-2+(p-1)\left[\frac{i-1}{2}\right]}, \mathbf{M}_{n ; n-1} \mathbf{L}_{n-1}^{p-2}, \mathbf{M}_{n ; r_{1}, \ldots, r_{j-1}, n-1} \mathbf{L}_{n-1}^{p-2+(p-1)\left[\frac{j-1}{2}\right]}\right\} \\
& \text { avec } 0 \leq s_{1}<\cdots<s_{i} \leq n-2 \text { et } 0 \leq r_{1}<\cdots<r_{j-1} \leq n-2 .
\end{aligned}
$$


Démonstration. - On a $\overline{\mathrm{T}}(D(n))=\mathcal{M}_{n}(p) \cap\left(H^{*} V_{n}\right)^{G_{n-1}}$. Posons $j=2 j^{\prime}+e$ avec $e \in\{0,1\}$. Comme

$$
\begin{aligned}
\mathbf{M}_{n-1 ; s_{1}, \ldots, s_{j}} \mathbf{L}_{n-1}^{p-2+(p-1)\left[\frac{j-1}{2}\right]} & =(-1)^{\left(\begin{array}{l}
j \\
2
\end{array}\right)} \widetilde{\mathbf{M}}_{n-1, s_{1}} \cdots \widetilde{\mathbf{M}}_{n-1, s_{j}} \mathfrak{e}_{n-1}^{e}, \\
\mathbf{M}_{n ; r_{1}, \ldots, r_{j-1}, n-1} \mathbf{L}_{n-1}^{p-2+(p-1)\left[\frac{j-1}{2}\right]} & =(-1)^{\left(\begin{array}{l}
j \\
2
\end{array}\right)} \frac{\widetilde{\mathbf{M}}_{n, r_{1}} \cdots \widetilde{\mathbf{M}}_{n, r_{j-1}} \widetilde{\mathbf{M}}_{n, n-1} \mathbf{L}_{n-1}^{p-2+(p-1)\left[\frac{j-1}{2}\right]}}{\mathbf{L}_{n}^{j-1+\frac{(p-3) j}{2}}} \\
& =(-1)^{\left(\begin{array}{c}
j \\
2
\end{array}\right)} \frac{\widetilde{\mathbf{M}}_{n, r_{1}} \cdots \widetilde{\mathbf{M}}_{n, r_{j-1}} \widetilde{\mathbf{M}}_{n, n-1} \mathbf{e}_{n-1}^{e}}{\mathbf{V}_{n}^{\frac{(p-1) j}{2}-1}},
\end{aligned}
$$

on déduit du lemme 6.7 et du théorème 6.14 que le module donné dans le théorème est contenu dans $\mathcal{M}_{n}(p) \cap\left(H^{*} V_{n}\right)^{G_{n-1}}$.

Montrons l'inclusion inverse. Soit $f \in \Lambda^{j}\left(x_{1}, \ldots, x_{n}\right) \otimes \mathbb{F}_{p}\left[y_{1}, \ldots, y_{n}\right]$ un élément de $\mathcal{M}_{n}(p) \cap\left(H^{*} V_{n}\right)^{G_{n-1}}$ de la forme

$$
f=\sum_{I} x_{I} f_{I}\left(y_{1}, \ldots, y_{n}\right) \text {. }
$$

Si $j=0$, alors $f$ est un élément de $\mathbb{F}_{p}\left[\mathbf{Q}_{n-1,0}, \ldots, \mathbf{Q}_{n-1, n-2}, \mathbf{V}_{n}\right]$. Supposons que $j>0$. D'après le lemme $6.8, y_{1}^{\frac{(p-1)(j-1)}{2}}$ divise $f_{I_{0}}$ avec $I_{0}=\{1, \ldots, j\}$. Comme $f$ est $G_{n-1}$-invariant, $f$ est aussi $\mathrm{GL}_{n-1}$-invariant. On déduit du lemme 6.9 que $\mathbf{L}_{n-1}^{p-2+(p-1)\left[\frac{j-1}{2}\right]}$ divise $f$ car

$$
\frac{(p-1)(j-1)}{2}+\overline{p-2-\frac{(p-1)(j-1)}{2}}=p-2+(p-1)\left[\frac{j-1}{2}\right] .
$$

Utilisant le théorème $6.14, f$ est un élément du module donné dans le théorème.

Corollaire 6.18. - $\left(D(n): L_{1}\right)$ u est un $\mathbb{F}_{p}\left[\mathbf{Q}_{n-1,0}, \ldots, \mathbf{Q}_{n-1, n-2}, \mathbf{V}_{n}^{p-1}\right]-m o-$ dule libre de base

$$
\begin{aligned}
& \left\{1, \mathbf{M}_{n-1 ; s_{1}, \ldots, s_{i}} \mathbf{L}_{n-1}^{p-2+(p-1)\left[\frac{i-1}{2}\right]}, \mathbf{M}_{n, n-1} \mathbf{L}_{n}^{p-2}, \quad \mathbf{M}_{n ; r_{1}, \ldots, r_{j-1}, n-1} \mathbf{L}_{n}^{p-2} \mathbf{L}_{n-1}^{(p-1)\left[\frac{j-1}{2}\right]}\right\} \\
& \text { avec } 0 \leq s_{1}<\cdots<s_{i} \leq n-2 \text { et } 0 \leq r_{1}<\cdots<r_{j-1} \leq n-2 .
\end{aligned}
$$

Démonstration. - L'action de $\mathrm{GL}_{1}$ sur $H^{*} \mathbb{Z} / p$ induit une action sur $\overline{\mathrm{T}}(D(n))$ à travers l'inclusion $\mathrm{GL}_{1} \hookrightarrow \mathrm{GL}_{n}$ donnée par $\lambda \mapsto \operatorname{diag}\left(1^{n-1}, \lambda\right)$. Le résultat se déduit facilement du théorème précédent.

6.4. Série de Poincaré de $\left(R_{s} \Sigma^{i} \mathbb{Z} / p: L_{1}\right) u_{\text {• }}$ - On note $P_{M}(t)$ la série de Poincaré du module instable $M$. Par définition des foncteurs $\Phi$ et $\Sigma$ [22], on a

$$
\begin{aligned}
P_{\Phi M}(t) & =P_{M^{+}}\left(t^{p}\right)+t^{2-p} P_{M^{-}}\left(t^{p}\right), \\
P_{\Phi \Sigma M}(t) & =t^{2} P_{M^{+}}\left(t^{p}\right)+t^{p} P_{M^{-}}\left(t^{p}\right) .
\end{aligned}
$$

Si $M=\left(R_{s} \Sigma^{i} \mathbb{Z} / p: L_{1}\right) u$, on écrit $P_{s, i}(t)\left(\operatorname{resp} . P_{s, i}^{+}(t), P_{s, i}^{-}(t)\right)$ pour $P_{M}(t)$ (resp. $\left.P_{M^{+}}(t), P_{M^{-}}(t)\right)$. 
Lemme 6.19. - Pour $s \geq 2$, on a

1. $\left(1-t^{2\left(p^{s-1}-1\right)}\right) P_{s, 0}^{+}(t)=P_{s-1,0}^{+}\left(t^{p}\right)+t^{p-2} P_{s-1,0}^{-}\left(t^{p}\right)$;

2. $\left(1-t^{2\left(p^{s-1}-1\right)}\right) P_{s, 0}^{-}(t)=t^{p-1} P_{s-1,0}^{-}\left(t^{p}\right)+t^{2 p^{s-1}-3} P_{s-1,0}^{+}\left(t^{p}\right)$.

Démonstration. - D'après le corollaire 6.18, le sous-espace des éléments de degré pair de $\left(D(s): L_{1}\right) u$ est le $\mathbb{F}_{p}\left[\mathbf{Q}_{s-1,0}, \ldots, \mathbf{Q}_{s-1, s-2}, \mathbf{V}_{s}^{p-1}\right]$-module libre de base

$$
\left\{1, \quad \mathbf{M}_{s-1 ; i_{1}, \ldots, i_{r}} \mathbf{L}_{s-1}^{p-2+(p-1)\left[\frac{r-1}{2}\right]}, \quad \mathbf{M}_{s ; j_{1}, \ldots, j_{k}, s-1} \mathbf{L}_{s}^{p-2} \mathbf{L}_{s-1}^{(p-1)\left[\frac{k}{2}\right]}\right\}
$$

où $\left\{i_{1}, \ldots, i_{r}\right\}$ avec $r$ pair (resp. $\left\{j_{1}, \ldots, j_{k}\right\}$ avec $k$ impair) est une partie ordonnée non-vide de $\{0, \ldots, s-2\}$. On notera $P_{s}^{+}(t)$ la série de Poincaré de l'espace vectoriel engendré par cette base. De même, le sous-espace des éléments de degré impair de $\left(D(s): L_{1}\right) u$ est le $\mathbb{F}_{p}\left[\mathbf{Q}_{s-1,0}, \ldots, \mathbf{Q}_{s-1, s-2}, \mathbf{V}_{s}^{p-1}\right]$-module libre de base

$$
\left\{\mathbf{M}_{s-1 ; i_{1}, \ldots, i_{r}} \mathbf{L}_{s-1}^{p-2+(p-1)\left[\frac{r-1}{2}\right]}, \quad \mathbf{M}_{s, s-1} \mathbf{L}_{s}^{p-2}, \quad \mathbf{M}_{s ; j_{1}, \ldots, j_{k}, s-1} \mathbf{L}_{s}^{p-2} \mathbf{L}_{s-1}^{(p-1)\left[\frac{k}{2}\right]}\right\}
$$

où $\left\{i_{1}, \ldots, i_{r}\right\}$ avec $r$ impair (resp. $\left\{j_{1}, \ldots, j_{k}\right\}$ avec $k$ pair) est une partie ordonnée non-vide de $\{0, \ldots, s-2\}$. On notera $P_{s}^{-}(t)$ la série de Poincaré de l'espace vectoriel engendré par cette base.

Le degré de $\mathbf{M}_{s-1 ; i_{1}, \ldots, i_{r}} \mathbf{L}_{s-1}^{p-2+(p-1)\left[\frac{r-1}{2}\right]}$ est

$$
2\left(p^{s-1}-1\right)\left(\left[\frac{r-1}{2}\right]+1\right)+\left(1-2 p^{i_{1}}\right)+\cdots+\left(1-2 p^{i_{r}}\right)
$$

et le degré de $\mathbf{M}_{s ; j_{1}, \ldots, j_{r}, s-1} \mathbf{L}_{s}^{p-2} \mathbf{L}_{s-1}^{(p-1)\left[\frac{r}{2}\right]}$ est

$$
2\left(p^{s-1}-1\right)\left[\frac{r}{2}\right]+\left(2 p^{s}-2 p^{s-1}-1\right)+\left(1-2 p^{j_{1}}\right)+\cdots+\left(1-2 p^{j_{r}}\right) .
$$

On en déduit, en posant $2 p^{I}=2 p^{i_{1}}+\cdots+2 p^{i_{r}}$ pour $I=\left(i_{1}, \ldots, i_{r}\right)$ et $2 p^{\varnothing}=0$, que

$$
\begin{aligned}
P_{s}^{+}(t) & =\sum_{\substack{I \subset\{0, \ldots, s-2\},|I| \text { pair }}} t^{p^{s-1}|I|-2 p^{I}}+\sum_{\substack{J \subset\{0, \ldots, s-2\},|J| \text { impair }}} t^{p^{s-1}(|J|-3)-2 p^{J}+2 p^{s}} \\
& =: A_{s}^{+}(t)+B_{s}^{+}(t) ; \\
P_{s}^{-}(t) & =\sum_{\substack{I \subset\{0, \ldots, s-2\},|I| \text { impair }}} t^{p^{s-1}(|I|+1)-2 p^{I}-1}+\sum_{\substack{J \subset\{0, \ldots, s-2\},|J| \text { pair }}} t^{p^{s-1}(|J|-2)-2 p^{J}+2 p^{s}-1} \\
= & : A_{s}^{-}(t)+B_{s}^{-}(t) .
\end{aligned}
$$

BULLETIN DE LA SOCIÉtÉ MATHÉMATIQUE DE FRANCE 
On a, en considérant si $I$ contient 0 ou non,

$$
\begin{aligned}
A_{s}^{+}(t)= & \sum_{\substack{I \subset\{0, \ldots, s-3\} \\
|I| \text { pair }}} t^{p\left(p^{s-2}|I|-2 p^{I}\right)}+\sum_{\substack{I \subset\{0, \ldots, s\},|I| \text { impair }}} t^{p\left(p^{s-2}(|I|+1)-2 p^{I}\right)-2} \\
= & A_{s-1}^{+}\left(t^{p}\right)+t^{p-2} A_{s-1}^{-}\left(t^{p}\right) ; \\
B_{s}^{+}(t)= & \sum_{\substack{J \subset\{0, \ldots, s-3\} \\
|J| \text { impair }}} t^{p\left(p^{s-2}(|J|-3)-2 p^{J}+2 p^{s-1}\right)} \\
& +\sum_{\substack{J \subset\{0, \ldots, s-3\},|J| \text { pair }}} t^{p\left(p^{s-2}(|J|-2)-2 p^{J}+2 p^{s-1}\right)-2} \\
= & B_{s-1}^{+}\left(t^{p}\right)+t^{p-2} B_{s-1}^{-}\left(t^{p}\right) .
\end{aligned}
$$

Il suit que

$$
P_{s}^{+}(t)=P_{s-1}^{+}\left(t^{p}\right)+t^{p-2} P_{s-1}^{-}\left(t^{p}\right) .
$$

De même,

$$
\begin{aligned}
A_{s}^{-}(t)= & \sum_{\substack{I \subset\{0, \ldots, s-3\},|I| \text { impair }}} t^{p\left(p^{s-2}(|I|+1)-2 p^{I}\right)-1}+\sum_{\substack{I \subset\{0, \ldots, s-2\},|I| \text { pair }}} t^{p\left(p^{s-1}(|I|+2)-2 p^{I}\right)-3} \\
= & t^{p-1} A_{s-1}^{-}\left(t^{p}\right)+t^{2 p^{s-1}-3} A_{s-1}^{+}\left(t^{p}\right) ; \\
B_{s}^{-}(t)= & \sum_{\substack{J \subset\{0, \ldots, s-3\},|J| \text { pair }}} t^{p\left(p^{s-2}(|J|-2)-2 p^{J}+2 p^{s-1}\right)-1} \\
& +\sum_{\substack{J \subset\{0, \ldots, s-3\},|J| \text { impair }}} t^{p\left(p^{s-2}(|J|-1)-2 p^{J}+2 p^{s-1}\right)-3} \\
= & t^{p-1} B_{s-1}^{-}\left(t^{p}\right)+t^{2 p^{s-1}-3} B_{s-1}^{+}\left(t^{p}\right) .
\end{aligned}
$$

D'où

$$
P_{s}^{+}(t)=t^{p-1} P_{s-1}^{-}\left(t^{p}\right)+t^{2 p^{s-1}-3} P_{s-1}^{+}\left(t^{p}\right) .
$$

On observe enfin que si l'on note $Q_{s}(t)$ la série de Poincaré de l'espace vectoriel $\mathbb{F}_{p}\left[\mathbf{Q}_{s-1,0}, \ldots, \mathbf{Q}_{s-1, s-2}, \mathbf{V}_{s}^{p-1}\right]$, alors

$$
Q_{s}(t)=\frac{1}{\left(1-t^{2\left(p^{s-1}-p^{0}\right)}\right) \cdots\left(1-t^{2\left(p^{s-1}-p^{s-2}\right)}\right)\left(1-t^{2 p^{s-1}(p-1)}\right)} .
$$

Il s'ensuit $\left(1-t^{2\left(p^{s-1}-1\right)}\right) Q_{s}(t)=Q_{s-1}(t)$. Le lemme est démontré.

Lemme 6.20 ([24, Définition-Proposition 4.5.1]). - Soient $s, i \geq 1$. Il existe une suite exacte courte des modules instables :

$$
0 \rightarrow \Sigma R_{s} \Sigma^{i} \mathbb{Z} / p \rightarrow \Sigma^{2} R_{s} \Sigma^{i-1} \mathbb{Z} / p \rightarrow \Phi \Sigma R_{s-1} \Sigma^{i-1} \mathbb{Z} / p \rightarrow 0 .
$$


Comme le foncteur $\left(-: L_{1}\right) u$ est exact et commute avec $\Phi$ et $\Sigma$ [22], il résulte du lemme que

$$
P_{s, i}(t)=t P_{s, i-1}(t)-t^{p-1} P_{s-1, i-1}^{-}\left(t^{p}\right)-t P_{s-1, i-1}^{+}\left(t^{p}\right) .
$$

Proposition 6.21. - Pour $s \geq 1$ et $i \geq 0$, on $a$

$$
P_{s, i}(t)= \begin{cases}t^{p^{s-1} i} P_{s, 0}(t) & \text { si } i \text { est pair } \\ t^{p^{s-1}(i-1)+1} P_{s, 0}^{-}(t)+t^{p^{s-1}(i-1)+2 p^{s-1}-1} P_{s, 0}^{+}(t) & \text { si } i \text { est impair } .\end{cases}
$$

Démonstration. - On fait une récurrence double sur $(i, s)$. On a rien à faire pour $i=0$. Pour $s=1$, on a $\left(R_{1} \Sigma^{i} \mathbb{Z} / p: L_{1}\right) u=\left(\Sigma^{i} M_{1}: L_{1}\right) u=$ $\Sigma^{i}\left(R_{1} \mathbb{Z} / p: L_{1}\right) u$ car le quotient de $\Sigma^{i} M_{1}$ par $R_{1} \Sigma^{i} \mathbb{Z} / p$ est un module fini. D'où $P_{1, i}(t)=t^{i} P_{1,0}(t)$.

Supposons que la formule de la proposition soit vérifiée pour tout $\left(s^{\prime}, i^{\prime}\right)<$ $(s, i)$ dans l'ordre lexicographique.

Supposons que $i$ est impair. On a

$$
\begin{aligned}
P_{s, i}(t)= & t P_{s, i-1}(t)-t^{p-1} P_{s-1, i-1}^{-}\left(t^{p}\right)-t P_{s-1, i-1}^{+}\left(t^{p}\right) \quad(\text { d'après }(1)) \\
= & t^{p^{s-1}(i-1)+1} P_{s, 0}(t)-t^{p^{s-1}(i-1)+p-1} P_{s-1,0}^{-}\left(t^{p}\right)-t^{p^{s-1}(i-1)+1} P_{s-1,0}^{-}\left(t^{p}\right) \\
& \quad(\text { par hypothèse de récurrence) } \\
= & t^{p^{s-1}(i-1)+1} P_{s, 0}(t)-t^{p^{s-1}(i-1)+1}\left(1-t^{2\left(p^{s-1}-1\right)}\right) P_{s, 0}^{+}(t)
\end{aligned}
$$

(d'après le lemme 6.19(1))

$$
=t^{p^{s-1}(i-1)+1} P_{s, 0}^{-}(t)+t^{p^{s-1}(i-1)+2 p^{s-1}-1} P_{s, 0}^{+}(t) \text {. }
$$

Supposons que $i$ est pair. On a

$$
\begin{aligned}
P_{s, i}(t)= & t P_{s, i-1}(t)-t^{p-1} P_{s-1, i-1}^{-}\left(t^{p}\right)-t P_{s-1, i-1}^{+}\left(t^{p}\right) \quad(\text { d'après }(1)) \\
= & t^{p^{s-1}(i-2)+2} P_{s, 0}^{-}(t)+t^{p^{s-1} i} P_{s, 0}^{+}(t)-t^{p^{s-1} i-1} P_{s-1,0}^{+}\left(t^{p}\right) \\
& \quad-t^{p^{s-1}(i-2)+1+p} P_{s-1,0}^{-}\left(t^{p}\right) \quad(\text { par hypothèse de récurrence) } \\
= & t^{p^{s-1}(i-2)+2} P_{s, 0}^{-}(t)+t^{p^{s-1}} i P_{s, 0}^{+}(t) \\
& -t^{p^{s-1}(i-2)+2}\left(1-t^{2\left(p^{s-1}-1\right)}\right) P_{s, 0}^{-}(t) \quad \text { (d'après le lemme 6.19(2)) } \\
= & t^{p^{s-1} i} P_{s, 0}(t) .
\end{aligned}
$$

La proposition est démontrée.

Pour $s \geq 1$ et $i \geq 0$, on note $c_{s, i}$ la connectivité de $\left(R_{s} \Sigma^{i} \mathbb{Z} / p: L_{1}\right) u$. BULLETIN DE LA SOCiÉTÉ MATHÉMATIQUE DE FRANCE 
Corollaire 6.22 . - On a

$$
c_{s, i}= \begin{cases}i & \text { si } s=1 \\ p^{s-1} i & \text { si } s \geq 2 \text { et } i \text { est pair } \\ p^{s-1} i+(p-2) p^{s-2} & \text { si } s \geq 2 \text { et } i \text { est impair. }\end{cases}
$$

En particulier, $c_{s, i} \geq p^{s-1} i$.

Démonstration. - Pour $s=1$, il est facile à voir que $c_{1, i}=i$.

Supposons que $s \geq 2$ et $i$ est pair. Il résulte de la proposition 6.21 que $c_{s, i}=p^{s-1} i$.

Supposons que $s \geq 2$ et $i$ est impair. On note $c_{s, i}^{+}, c_{s, i}^{-}$respectivement la connectivité de $\left(R_{s} \Sigma^{i} \mathbb{Z} / p: L_{1}\right)_{\mathcal{u}}^{+},\left(R_{s} \Sigma^{i} \mathbb{Z} / p: L_{1}\right)_{\mathcal{u}}^{-}$. D'après la proposition 6.21 , on a

$$
\begin{aligned}
& c_{s, i}^{+}=p^{s-1}(i-1)+1+c_{s, 0}^{-} \\
& c_{s, i}^{-}=p^{s-1}(i+1)-1 .
\end{aligned}
$$

D'après le lemme 6.19 , on a

$$
c_{s, 0}^{-}=\min \left(p-1+p c_{s-1,0}^{-} ; 2 p^{s-1}-3\right)
$$

pour tout $s \geq 2$. De plus, il est facile à vérifier que $c_{1,0}^{-}=2 p-3$. Par récurrence, on peut montrer que $c_{s, 0}^{-}=2 p^{s-1}-2 p^{s-2}-1$ pour $s \geq 2$. Il suit que

$$
c_{s, i}^{+}=p^{s-1}(i+1)-2 p^{s-2} .
$$

Comme $c_{s, i}=\min \left(c_{s, i}^{+}, c_{s, i}^{-}\right)$, on obtient $c_{s, i}=p^{s-1} i+(p-2) p^{s-2}$. Le corollaire est démontré.

Corollaire 6.23. - Si $s+t \geq 1$, la connectivité de $\left(D_{s} \Sigma^{t} \mathbb{Z} / p: L_{1}\right)$ u est supérieure ou égale à $p^{s-1}(s+t-1)+1$.

Démonstration. - On a $\left(D_{s} \Sigma^{t} \mathbb{Z} / p: L_{1}\right) u \cong \Sigma\left(R_{s} \Sigma^{s+t-1} \mathbb{Z} / p: L_{1}\right) u$. Le corollaire se déduit du corollaire précédent.

\section{BIBLIOGRAPHIE}

[1] J. F. Adams, J. H. Gunawardena \& H. Miller - « The Segal conjecture for elementary abelian p-groups », Topology 24 (1985), p. 435-460.

[2] G. Z. Arone \& W. G. DWyer - «Partition complexes, Tits buildings and symmetric products », Proc. London Math. Soc. 82 (2001), p. 229-256.

[3] G. Z. Arone \& M. Mahowald - « The Goodwillie tower of the identity functor and the unstable periodic homotopy of spheres », Invent. Math. 135 (1999), p. 743-788. 
[4] H. E. A. Campbell \& P. S. Selick - « Polynomial algebras over the Steenrod algebra », Comment. Math. Helv. 65 (1990), p. 171-180.

[5] H. Cartan \& S. Eilenberg - Homological algebra, Princeton Univ. Press, 1956.

[6] L. E. DiCKSON - «A fundamental system of invariants of the general modular linear group with a solution of the form problem », Trans. Amer. Math. Soc. 12 (1911), p. 75-98.

[7] T. G. Goodwillie - « Calculus. III. Taylor series », Geom. Topol. 7 (2003), p. 645-711.

[8] N. D. H. Hai, L. Schwartz \& T. N. NAM - « La fonction génératrice de Minc et une "conjecture de Segal" pour certains spectres de Thom », Adv. Math. 225 (2010), p. 1431-1460.

[9] N. J. KUHN - « The modular Hecke algebra and Steinberg representation of finite Chevalley groups », J. Algebra 91 (1984), p. 125-141.

[10] _ « Chevalley group theory and the transfer in the homology of symmetric groups », Topology 24 (1985), p. 247-264.

[11] _ « The rigidity of $L(n) »$, in Algebraic topology (Seattle, Wash., 1985), Lecture Notes in Math., vol. 1286, Springer, 1987, p. 286-292.

[12] J. LANNES - «Sur les espaces fonctionnels dont la source est le classifiant d'un p-groupe abélien élémentaire », Publ. Math. I.H.É.S. 75 (1992), p. $135-244$.

[13] J. LAnnes \& L. Schwartz - « Sur la structure des $A$-modules instables injectifs », Topology 28 (1989), p. 153-169.

[14] J. Lannes \& S. Zarati - «Sur les $\mathscr{U}$-injectifs », Ann. Sci. École Norm. Sup. 19 (1986), p. 303-333.

[15] C. LÖFWALL - «On the subalgebra generated by the one-dimensional elements in the Yoneda Ext-algebra », Lecture Notes in Math. 1183 (1986), p. 291-338.

[16] P. A. Minh \& V. T. TÙnG - «Modular invariants of parabolic subgroups of general linear groups », J. Algebra 232 (2000), p. 197-208.

[17] S. A. Mitchell \& S. B. Priddy - « Stable splittings derived from the Steinberg module », Topology 22 (1983), p. 285-298.

[18] H. MÙI - « Modular invariant theory and cohomology algebras of symmetric groups », J. Fac. Sci. Univ. Tokyo Sect. IA Math. 22 (1975), p. 319369 .

[19] _ « Cohomology operations derived from modular invariants », Math. Z. 193 (1986), p. 151-163.

[20] A. Polishrhuk \& L. Positselski - Quadratic algebras, University Lecture Series, vol. 37, Amer. Math. Soc., 2005. 
[21] S. B. PrIDdy - « Koszul resolutions », Trans. Amer. Math. Soc. 152 (1970), p. 39-60.

[22] L. SchWARTZ - Unstable modules over the Steenrod algebra and Sullivan's fixed point set conjecture, Chicago Lectures in Mathematics, University of Chicago Press, 1994.

[23] R. Steinberg - « Prime power representations of finite linear groups », Canad. J. Math. 8 (1956), p. 580-591.

[24] S. ZARATi - « Dérivés du foncteur de déstabilisation en caractéristique impaire et applications », thèse de doctorat, Université Paris-Sud (Orsay), 1984.

TOME $140-2012-\mathrm{N}^{\circ} 2$ 